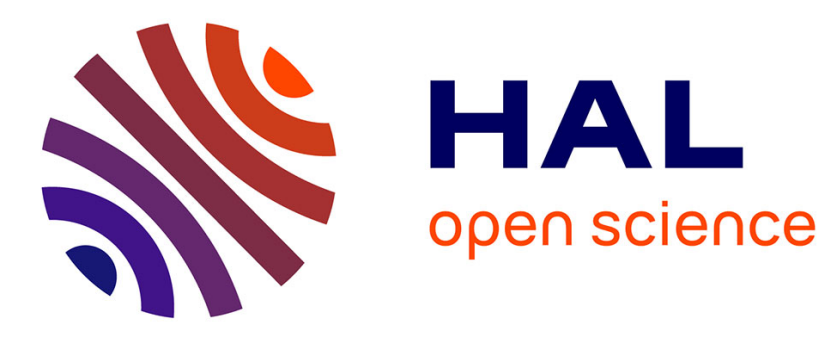

\title{
Clathrin packets move in slow axonal transport and deliver functional payloads to synapses
}

Archan Ganguly, Rohan Sharma, Nicholas P Boyer, Florian Wernert, Sébastien Phan, Daniela Boassa, Leonardo Parra, Utpal Das, Ghislaine Caillol, Xuemei Han, et al.

\section{To cite this version:}

Archan Ganguly, Rohan Sharma, Nicholas P Boyer, Florian Wernert, Sébastien Phan, et al.. Clathrin packets move in slow axonal transport and deliver functional payloads to synapses. Neuron, 2021, 109 (18), pp.2884 - 2901.e7. 10.1016/j.neuron.2021.08.016 . hal-03374115

\section{HAL Id: hal-03374115 https://hal.science/hal-03374115}

Submitted on 12 Oct 2021

HAL is a multi-disciplinary open access archive for the deposit and dissemination of scientific research documents, whether they are published or not. The documents may come from teaching and research institutions in France or abroad, or from public or private research centers.
L'archive ouverte pluridisciplinaire HAL, est destinée au dépôt et à la diffusion de documents scientifiques de niveau recherche, publiés ou non, émanant des établissements d'enseignement et de recherche français ou étrangers, des laboratoires publics ou privés. 


\section{Neuron}

\section{Clathrin packets move in slow axonal transport and deliver functional payloads to synapses}

\section{Graphical abstract}

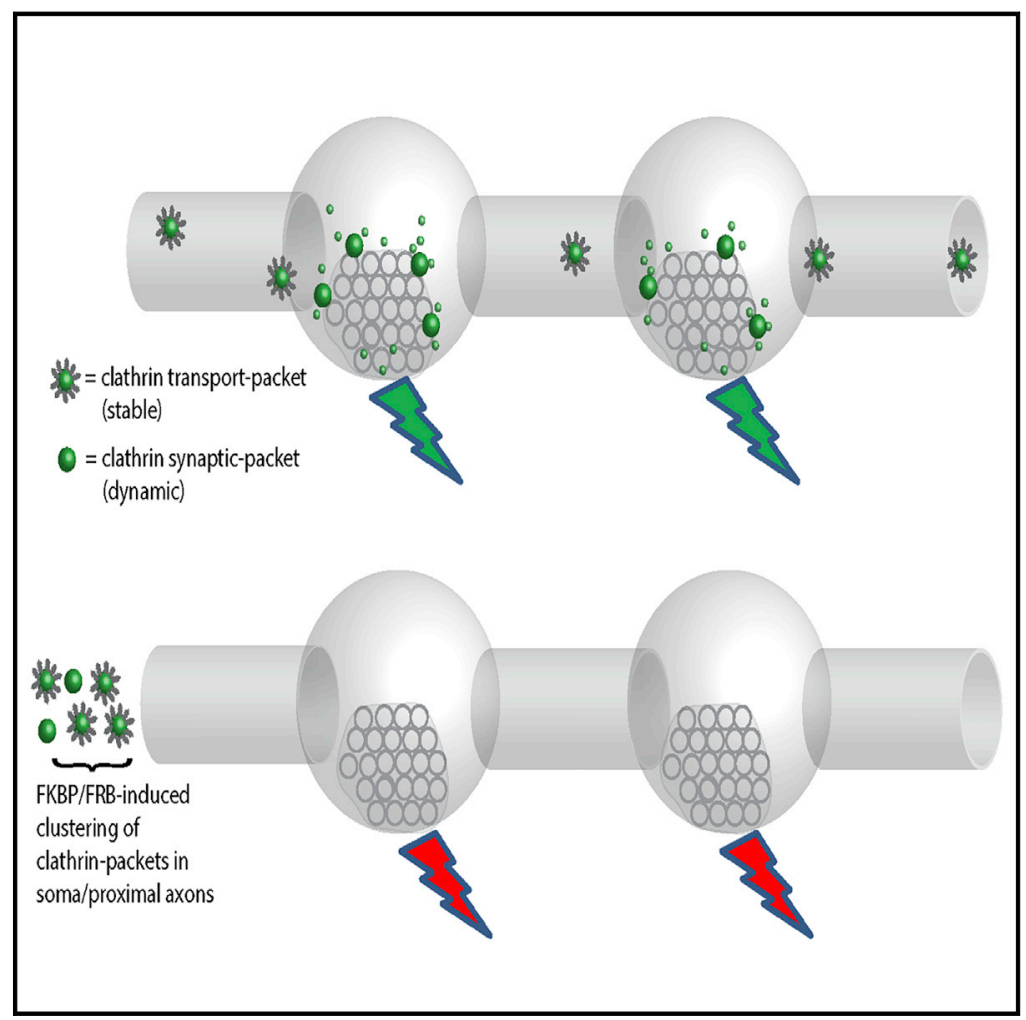

\section{Highlights}

- Clathrin moves as stable "transport packets" in axons with overall anterograde bias

- Stable clathrin packets switch to a dynamic state upon reaching synapses

- Synaptic clathrin packets are radially organized around synaptic vesicle clusters

- Depletion of synaptic clathrin packets impairs synaptic vesicle recycling

\section{Authors}

Archan Ganguly, Rohan Sharma, Nicholas P. Boyer, ..., Mark H. Ellisman, Christophe Leterrier, Subhojit Roy

\section{Correspondence}

sroy@ucsd.edu

\section{In brief}

Clathrin helps maintain synaptic homeostasis, but it's unclear how it gets to synapses. Ganguly and Sharma et al. show that clathrin assembles into stable transport packets in axons that are trapped upon reaching synapses, where packets radially organize around synaptic vesicle clusters. Synaptic clathrin packets are dynamic, and their depletion impairs synaptic function. 


\title{
Article
}

\section{Clathrin packets move in slow axonal transport and deliver functional payloads to synapses}

\author{
Archan Ganguly, ${ }^{1,6,7}$ Rohan Sharma, ${ }^{1,7}$ Nicholas P. Boyer, ${ }^{1}$ Florian Wernert, ${ }^{2}$ Sébastien Phan, ${ }^{3,4}$ Daniela Boassa, ${ }^{3,4}$ \\ Leonardo Parra, ${ }^{1}$ Utpal Das, ${ }^{1,3}$ Ghislaine Caillol, ${ }^{2}$ Xuemei Han, ${ }^{5}$ John R. Yates III, ${ }^{5}$ Mark H. Ellisman, ${ }^{3,4}$ \\ Christophe Leterrier, ${ }^{2}$ and Subhojit Roy ${ }^{1,3,8, *}$ \\ ${ }^{1}$ Department of Pathology, University of California, San Diego, La Jolla, CA, USA \\ ${ }^{2}$ Aix Marseille Université, CNRS, INP UMR7051, NeuroCyto, Marseille, France \\ 3Department of Neurosciences, University of California, San Diego, La Jolla, CA, USA \\ ${ }^{4}$ National Center for Microscopy and Imaging Research, University of California, San Diego, La Jolla, CA, USA \\ ${ }^{5}$ Department of Cell Biology, The Scripps Research Institute, La Jolla, CA, USA \\ 6Present address: Department of Pharmacology and Physiology, University of Rochester Medical Center, Rochester, NY, USA \\ ${ }^{7}$ These authors contributed equally \\ 8 Lead contact \\ *Correspondence: sroy@ucsd.edu \\ https://doi.org/10.1016/j.neuron.2021.08.016
}

\section{SUMMARY}

In non-neuronal cells, clathrin has established roles in endocytosis, with clathrin cages enclosing plasma membrane infoldings, followed by rapid disassembly and reuse of monomers. However, in neurons, clathrin is conveyed in slow axonal transport over days to weeks, and the underlying transport/targeting mechanisms, mobile cargo structures, and even its precise presynaptic localization and physiologic role are unclear. Combining live imaging, photobleaching/conversion, mass spectrometry, electron microscopy, and super-resolution imaging, we found that unlike in dendrites, where clathrin cages rapidly assemble and disassemble, in axons, clathrin and related proteins organize into stable "transport packets" that are unrelated to endocytosis and move intermittently on microtubules, generating an overall slow anterograde flow. At synapses, multiple clathrin packets abut synaptic vesicle (SV) clusters, and clathrin packets also exchange between synaptic boutons in a microtubule-dependent "superpool." Within synaptic boundaries, clathrin is surprisingly dynamic, continuously exchanging between local clathrin assemblies, and its depletion impairs SV recycling. Our data provide a conceptual framework for understanding clathrin trafficking and presynaptic targeting that has functional implications.

\section{INTRODUCTION}

The cytosolic protein clathrin is an established player in endocytosis. During this process, soluble clathrin is recruited to the inner plasma membrane, forming clathrin-coated pits and vesicles. After vesicle internalization, the clathrin coat is rapidly removed by uncoating proteins, and the released clathrin monomers are reused for subsequent rounds of endocytosis. Clathrin-mediated endocytosis (CME) has been studied for over forty years, and a host of adapters and regulators are known. Clathrin also plays roles in sorting of intracellular membranes, although this is less studied (Jung and Haucke, 2007; Dittman and Ryan, 2009; McMahon and Boucrot, 2011; Traub and Bonifacino, 2013).

In neurons, clathrin synthesized in cell bodies is transported into axons, enriching at presynapses, and previous in vivo pulse-chase radiolabeling studies have established that clathrin is conveyed in slow axonal transport, taking days to weeks in reaching axon terminals (Black et al., 1991; de Waegh and Brady, 1989; Elluru et al., 1995; Garner and Lasek, 1981; Gower and Tytell, 1987). Slow transport is a poorly defined rate-class carrying cytosolic (or soluble) and cytoskeletal proteins to the axon tips and presynaptic terminals, unlike fast vesicular cargoes that are rapidly transported in minutes to hours (for review, see Maday et al., 2014; Roy, 2014, 2020). Although radiolabeling techniques established the overall movement of clathrin in slow transport, these methods could not visualize the movement, and many questions remain.

How can clathrin undergo organized slow axonal transport, given the ephemeral nature of cytoplasmic clathrin assemblies? Indeed, previous studies in cultured hippocampal neurons showed rapid on/off behavior of GFP:clathrin puncta in dendrites, lasting only for seconds, consistent with CME (Blanpied et al., 2002; Rosendale et al., 2017). While clathrin may organize into longerlasting assemblies for slow transport, no such structures have been described. How is clathrin targeted to presynapses and 
retained there? What is its nanoscale organization at synapses? What is the dynamic behavior of clathrin at the synapse, and can this offer insight into function? Though clathrin is involved in retrieving SVs from the presynaptic plasma membrane (Heuser and Reese, 1973; Royle and Lagnado, 2010; Saheki and De Camilli, 2012), newer studies argue that clathrin acts downstream of endocytosis - in the regeneration of new SVs (Chanaday et al., 2019; Chanaday and Kavalali, 2018; Milosevic, 2018; Watanabe et al., 2014) - and a better understanding of the precise localization and dynamic behavior of clathrin may offer a fresh perspective.

Recent studies from us and others using cultured neurons as a model system to visualize and manipulate slow axonal transport have begun to offer some answers about mechanisms (Chakrabarty et al., 2019; Ganguly et al., 2017; Scott et al., 2011; Tang et al., 2013; Twelvetrees et al., 2016). Here, we report previously unknown long-lasting axonal assemblies of clathrin and associated proteins that are unrelated to endocytosis and specialized for cargo delivery. We also found a striking radial organization of clathrin around-but not within-SV clusters, and a microtubule-dependent clathrin superpool that may be distinct from the known actin-dependent SV superpool (Darcy et al., 2006). Though the transported axonal clathrin packets are stable, surprisingly, they become dynamic upon entering synaptic boundaries-exchanging clathrin molecules with local synaptic clathrin-and depletion of clathrin packets from boutons impairs SV recycling. Collectively, the data advocate new models for trafficking and targeting of clathrin and related proteins, with implications for synaptic function.

\section{RESULTS}

Discrete clathrin transport packets move intermittently in slow axonal transport

Although the overall appearance of clathrin puncta in cultured neurons seems similar in somatodendritic and axonal compartments by immunostaining (Figure $1 A$ ), their dynamics were different. To visualize clathrin in living neurons, we used GFP (or mCherry) tagged to the $\mathrm{N}$ terminus of clathrin light chain $\mathrm{A}$ (CLC), a structurally and functionally active fusion protein (Gaidarov et al., 1999) that has been used in other studies (Blanpied et al., 2002; Mueller et al., 2004; Pelassa et al., 2014; Rosendale et al., 2017; Zhao and Keen, 2008). All live imaging of axons was performed between 8 and 10 days in vitro (DIV), and synapses were imaged between 13 and 21 DIV - a time when synapses are established in our cultures. As reported previously (Blanpied et al., 2002; Rosendale et al., 2017), GFP:CLC showed largely on/off dynamics in dendrites, reflecting transient recruitment of clathrin molecules to coated pits (see kymographs in Figure 1B; also see Video S1). However, axonal GFP:CLC particles were long-lasting, and $\sim 40 \%$ were motile, moving with an overall anterograde bias (Figures 1C-1E; see also Figure S1A and Video S2). Where visible, moving clathrin particles did not colocalize with transported vesicles (Figure S1B). To distinguish moving axonal clathrin structures from dendritic clathrin-coated pits and other endocytosis-related clathrin structures, here we call them clathrin "transport packets."

Previously, we developed an assay to visualize the anterogradely biased transport of cytosolic cargoes moving in slow transport ("intensity-center shift assay"; see Roy et al., 2011; Scott et al., 2011). Here, cultured neurons are transfected with photoactivatable (or photoconvertible) probes tagged to the cytosolic protein of interest, and a discrete region of interest (ROI) in the axon shaft is photoactivated/photoconverted (schematic in Figure 1F). Thereafter, a line is drawn along the long axis of the axon, including the photoactivated/photoconverted ROI, and the intensity center (or centroid) shift of the fluorescence in the $\mathrm{ROI}$ is monitored over time. While untagged photoactivatable GFP (PAGFP) rapidly diffuses without any centroid shift, cytosolic proteins disperse with a slow anterogradely biased centroid shift, consistent with slow transport (Chakrabarty et al., 2019; Ganguly et al., 2017; Scott et al., 2011; Tang et al., 2012, 2013; Twelvetrees et al., 2016). A kymograph from these experiments is shown in Figure $1 \mathrm{G}$, along with the ensuing raw and ensemble data (Figures $1 \mathrm{H}$ and $1 \mathrm{I}$ ). Note that PAGFP:CLC-but not soluble, untagged PAGFP - has an overall anterograde bias with a predicted average rate of $\sim 0.006 \mu \mathrm{m} / \mathrm{s}$ or $\sim 0.5 \mathrm{~cm} /$ day, in line with overall rates of slow transport in radiolabeling studies (Garner and Lasek, 1981). Similar results were obtained with a photoconversion assay, in which we first visualized green Dendra2:CLC puncta in axons and then photoconverted them to red (Figure 1J). Collectively, the data indicate that the intermittent movement of axonal clathrin transport packets generates an overall anterograde bias of the clathrin population, conveyed at rates consistent with slow axonal transport.

\section{Motility of clathrin transport packets is independent of endocytosis}

The persistent and biased movement of the transport packets suggest that clathrin is conveyed as a unique structure that is specialized for axonal transport, with no role in endocytosis. To test this idea, we asked if inhibition of endocytosis would also affect the motility of axonal transport packets. First, we globally suppressed endocytosis in cultured neurons using Dynasore, a small-molecule inhibitor of dynamin (Das et al., 2013; Macia et al., 2006). We also specifically inhibited clathrin-dependent endocytosis with $\mathrm{CHC}-\mathrm{T} 7-\mathrm{Hub}$, a clathrin heavy-chain dominant-negative mutant known to disrupt clathrin-dependent endocytosis (Bennett et al., 2001). As reported previously in non-neuronal cells, the CHC-T7-Hub mutant attenuated receptor-mediated endocytosis in cultured hippocampal neurons (Figures S2A and S2B). The on/off dynamics of GFP:CLC in dendrites was essentially abolished in neurons treated with Dynasore (Figure 2A) or transfected with the CHC-T7-Hub mutant (Figures S2C and S2D). Interestingly, however, neither Dynasore nor the CHC-T7-Hub mutant had any effect on the mobility of axonal clathrin transport packets (Figures 2B-2D). Since the motility of individual clathrin transport packets generates the overall anterogradely biased flow of the axonal clathrin population (see Figure $1 \mathrm{H}$ ), a prediction is that endocytosis inhibition would not interfere with the slow transport of clathrin. Indeed, neither Dynasore nor the CHC-T7-Hub mutant had any effect on the biased transit of PAGFP:CLC in axons (Figure 2E). Previous studies suggest that the slow transport of synapsin and dynein is microtubule dependent (Scott et al., 2011; Twelvetrees et al., 2016). Low levels of the microtubule-disrupting drug nocodazole (at a concentration that blocks vesicle transport; see 
A
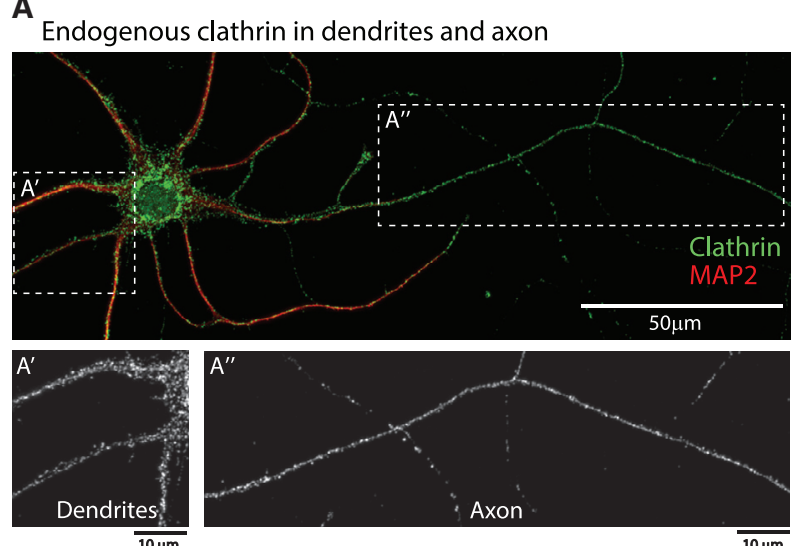

F Strategy to visualize slow axonal transport

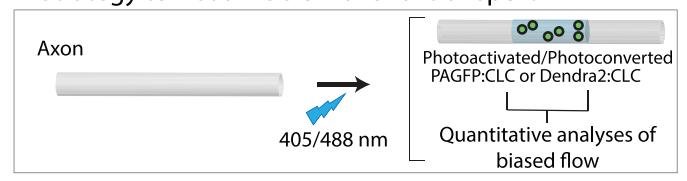

G Photoactivation in axon (PAGFP:CLC)

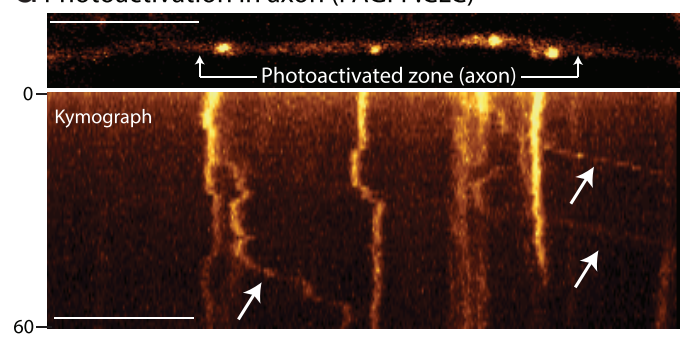

B GFP:CLC in dendrite

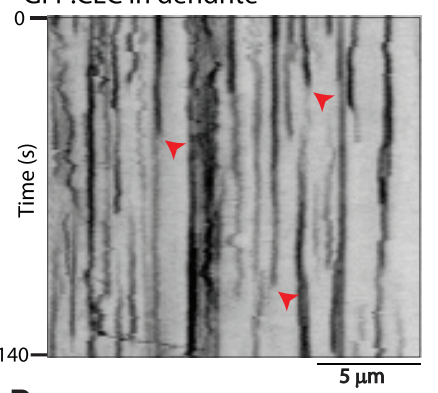

D
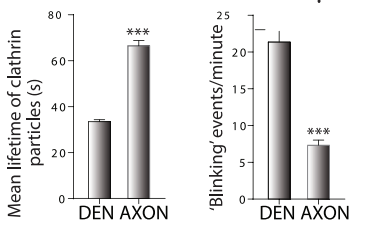

I

H

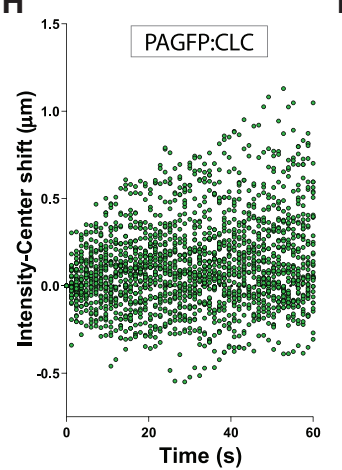

C

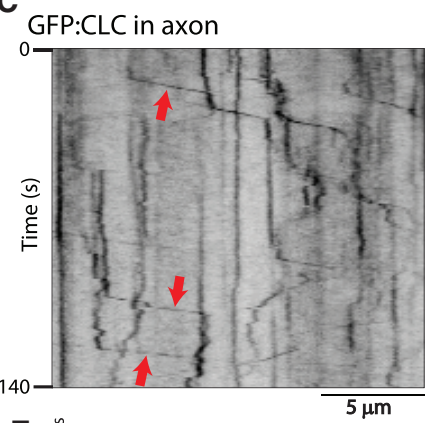

E
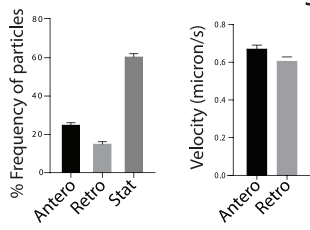

J Clathrin photoconversion

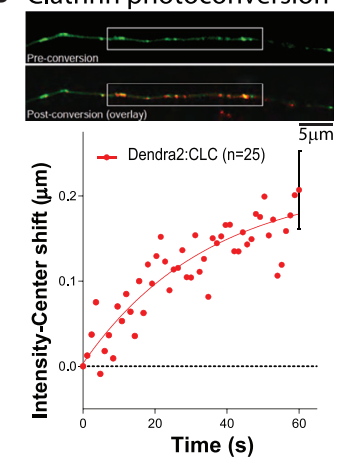

Figure 1. Differential dynamics of clathrin in dendrites and axons

(A) Clathrin and MAP2 immunostaining in cultured hippocampal neurons. Note punctate clathrin in both somatodendritic ( $\left.\mathrm{A}^{\prime}\right)$ and axonal (A") compartments.

(B) Kymograph of GFP:CLC in a dendrite. Note abrupt appearance and disappearance of fluorescence (red arrowheads), indicating assembly/disassembly of clathrin in coated pits (CME). Scale bars, $5 \mu \mathrm{m}$.

(C) Kymograph of GFP:CLC in an axon. Note rapid, infrequent, and anterogradely biased movement of clathrin particles (some marked by red arrows; more examples in Figure S1A). Scale bars, $5 \mu \mathrm{m}$.

(D) Mean lifetimes of GFP:CLC particles as determined from kymographs were significantly higher in axons, while "blinking events"-representing clathrin assembly/disassembly and receptor-mediated endocytosis - are much lower in axons, compared to dendrites (1,113 particles from 22 dendrites and 403 particles from 41 axons were analyzed; data were pooled from 3 independent experiments; ${ }^{* *} p<0.0001$ ).

(E) About $40 \%$ of the axonal GFP:CLC particles were mobile, with a larger fraction of particles moving anterogradely ( 460 anterograde and 263 retrograde events were analyzed from 41 axons; data were combined from 3 independent experiments).

(F) Strategy for analyzing biased axonal transport of clathrin in axons (see Results for details).

(G) Kymograph from a photoactivation (PAGFP:CLC) experiment. Note anterograde movement of photoactivated clathrin particles (arrows). Scale bars, $10 \mu \mathrm{m}$. (H) Raw data of intensity-center shifts from all PAGFP:CLC experiments in axons. Note anterograde drift of datapoints (38 neurons from 3 separate cultures were analyzed).

(I) Ensemble data showing mean intensity-center shift of PAGFP:CLC and PAGFP-only in axons, with fitted curves. While there is an overall anterograde bias of PAGFP:CLC, there is no bias with untagged PAGFP-only (10 neurons from 2 separate cultures were analyzed for PAGFP-only).

(J) Neurons were transfected with Dendra2:CLC, axonal clathrin particles were identified by green fluorescence, and intensity-center shifts of photoconverted (red) particles were analyzed. Note anterograde shift of the particles ( 25 neurons from 3 separate cultures were analyzed). Scale bars, $5 \mu \mathrm{m}$.

Figure S2F) also inhibited movement of clathrin transport packets (Figure 2F). Taken together, the data indicate that axonal clathrin transport packets are unique conveyance structures, unrelated to endocytosis.

Structure of axonal clathrin transport packets What do the transport packets look like? To visualize the ultrastructure of axonal clathrin, we tagged clathrin to APEX, an engineered peroxidase that acts as an electron microcopy (EM) tag by catalyzing the polymerization and local deposition of diaminobenzidine (DAB), subsequently recruiting electron-dense osmium for EM contrast (Martell et al., 2012). Cultured neurons were transfected with APEX-GFP:CLC and fixed and incubated with DAB and $\mathrm{H}_{2} \mathrm{O}_{2}$ (see schematic in Figure $3 \mathrm{~A}$ and STAR methods). Transfected neurons were visualized by GFP fluorescence and DAB staining, dendrites and axons were identified by morphology, and the same neurons and processes were visualized by EM tomography (see Figure S3 and STAR methods). As 
A GFP:CLC in dendrites after endocytosis inhibition

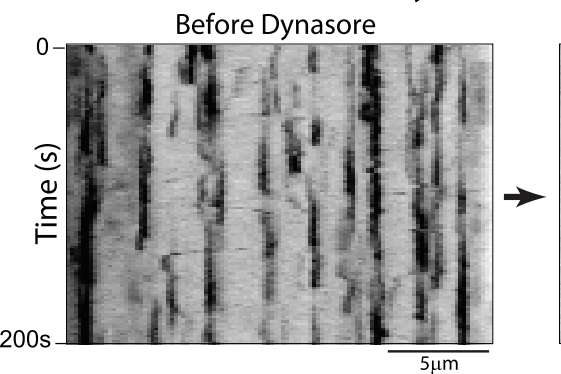

B mCherry:CLC in axons after Dynasore

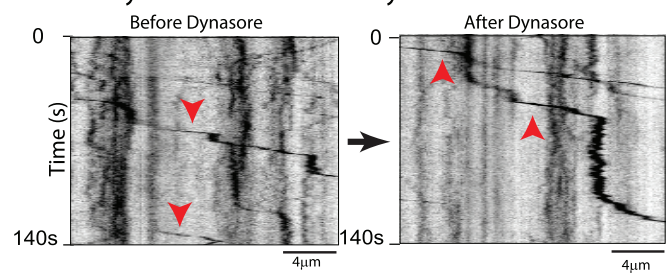

C mCherry:CLC in axons after T7-Hub mutant

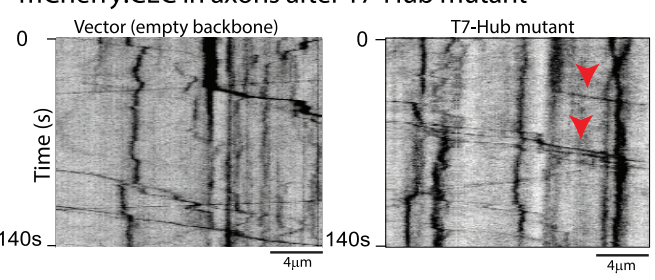

After Dynasore
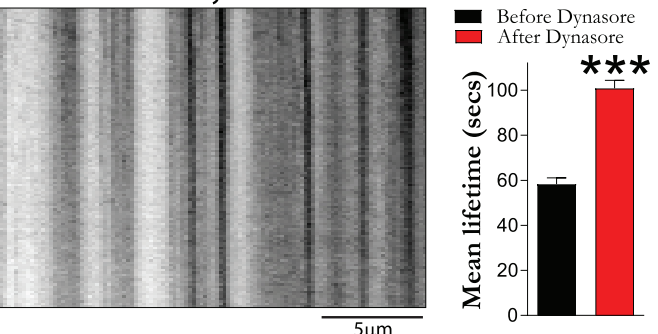

D

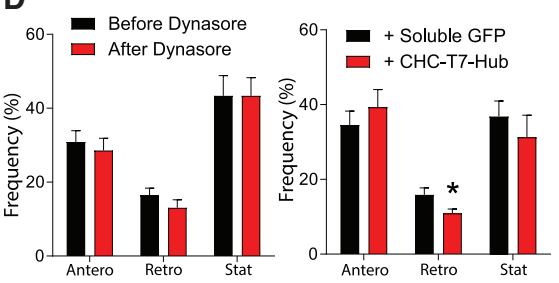

E
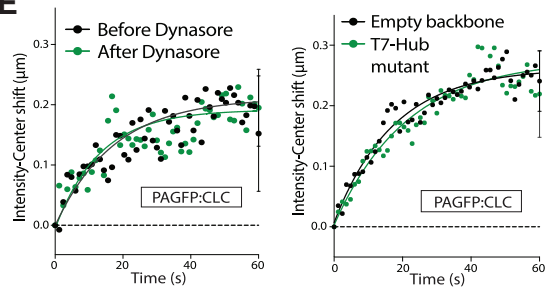

F GFP:CLC in axons after Nocodazole
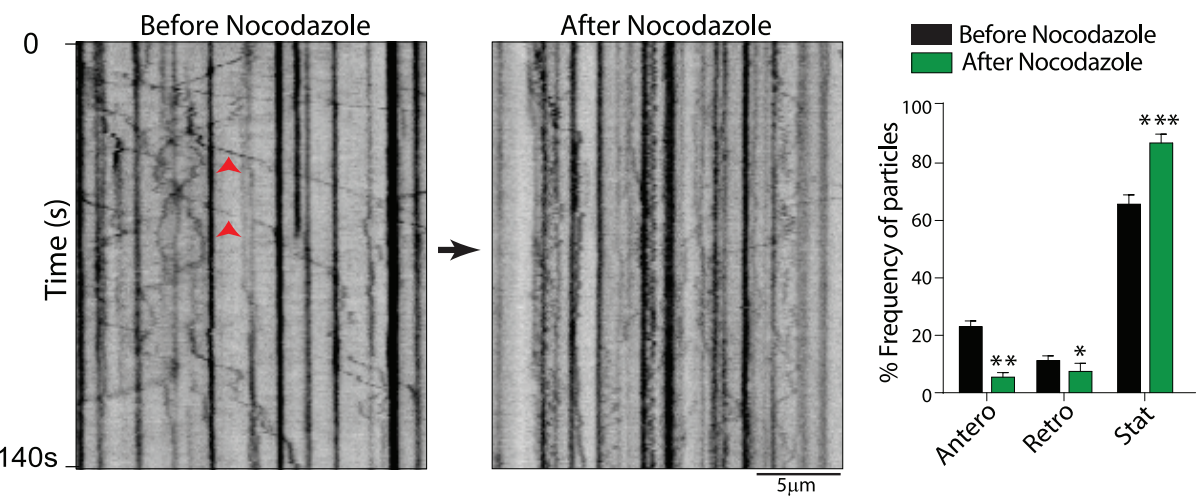

Figure 2. Motility of axonal clathrin transport packets is independent of endocytosis, but dependent on microtubules

(A) Kymographs of GFP:CLC from a dendrite before and after endocytosis inhibition (Dynasore). Note that blinking events-reflecting endocytosis-are abolished after drug treatment. GFP:CLC fluorescence lifetimes are quantified on the right ( 150-250 particles from 10 neurons were analyzed; data from three separate cultures; $\left.{ }^{* \star *} \mathrm{p}<0.0001\right)$. Scale bars, $5 \mu \mathrm{m}$.

(B) Kymographs of mCherry:CLC from same axon before and after Dynasore. Note that vectorial movement of clathrin transport packets (some marked by arrowheads) continues after drug treatment. Scale bars, $4 \mu \mathrm{m}$.

(C) Kymographs of axonal mCherry:CLC from neurons transfected with a dominant-negative clathrin mutant (T7-Hub) that specifically interferes with clathrindependent endocytosis (see Figure S2) or control. Note that vectorial movement of clathrin transport packets (some marked by arrowheads) are similar in the two groups. Scale bars, $4 \mu \mathrm{m}$.

(D) Quantification of all mCherry:CLC axonal transport data, with and without endocytosis inhibition, global or clathrin dependent. ( 140-170 motile particles were analyzed from 10-12 neurons; data from $2-3$ separate cultures; ${ }^{*} p<0.01$.)

(E) Ensemble intensity-center shift data (PAGFP:CLC slow transport assay) from neurons treated with Dynasore or co-transfected with the T7-Hub mutant. Note that inhibiting global-or clathrin-dependent-endocytosis has no effect on the slow anterograde bias of the clathrin population in axons (20-30 axons from two and four separate cultures were analyzed).

(F) Kymographs of GFP:CLC dynamics from the same axon before and after treatment with $10 \mu \mathrm{g} / \mathrm{mL}$ nocodazole for 30 min; quantification on right. Note that motility of the clathrin transport packets is attenuated after drug treatment. Scale bars, $5 \mu \mathrm{m}\left({ }^{\star \star} \mathrm{p}<0.01 .{ }^{\star \star} \mathrm{p}<0.001,{ }^{\star \star \star} \mathrm{p}<0.0001\right)$. 
A APEX-EM tomography

Transfect
neurons
with
APEX-GFP:CLC $\rightarrow \begin{gathered}\text { Fix and } \\ \text { expose to } \\ \text { DAB+ H2O2 }\end{gathered} \rightarrow \begin{gathered}\text { Embed/section } \\ \text { transfected } \\ \text { neurons and } \\ \text { 3D EM tomography }\end{gathered}$

B DAB staining in a transfected neuron

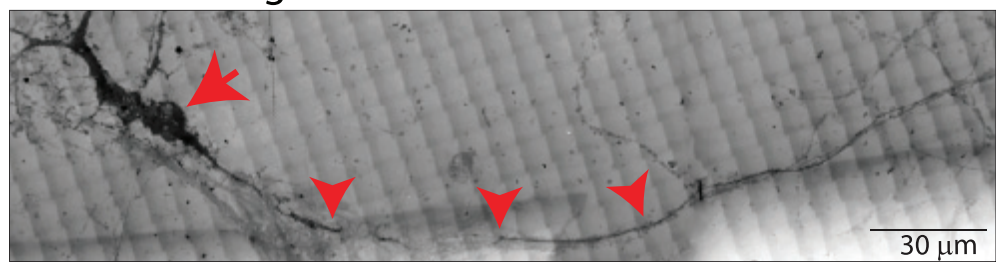

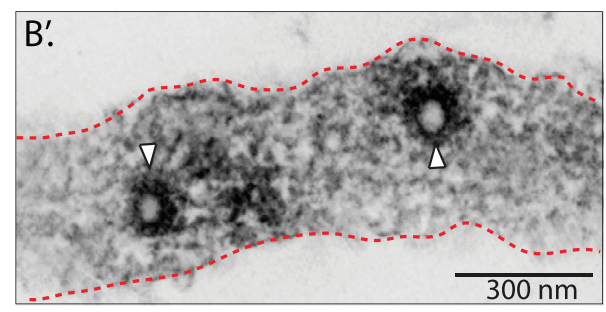
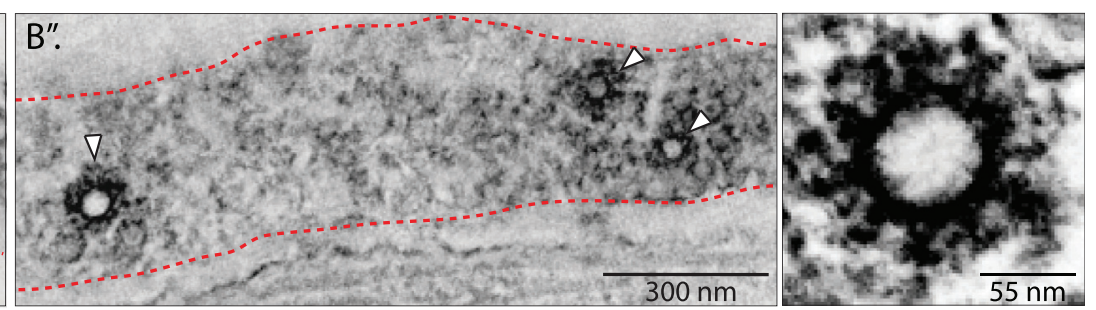

\section{DNA-PAINT of endogenous axonal clathrin}
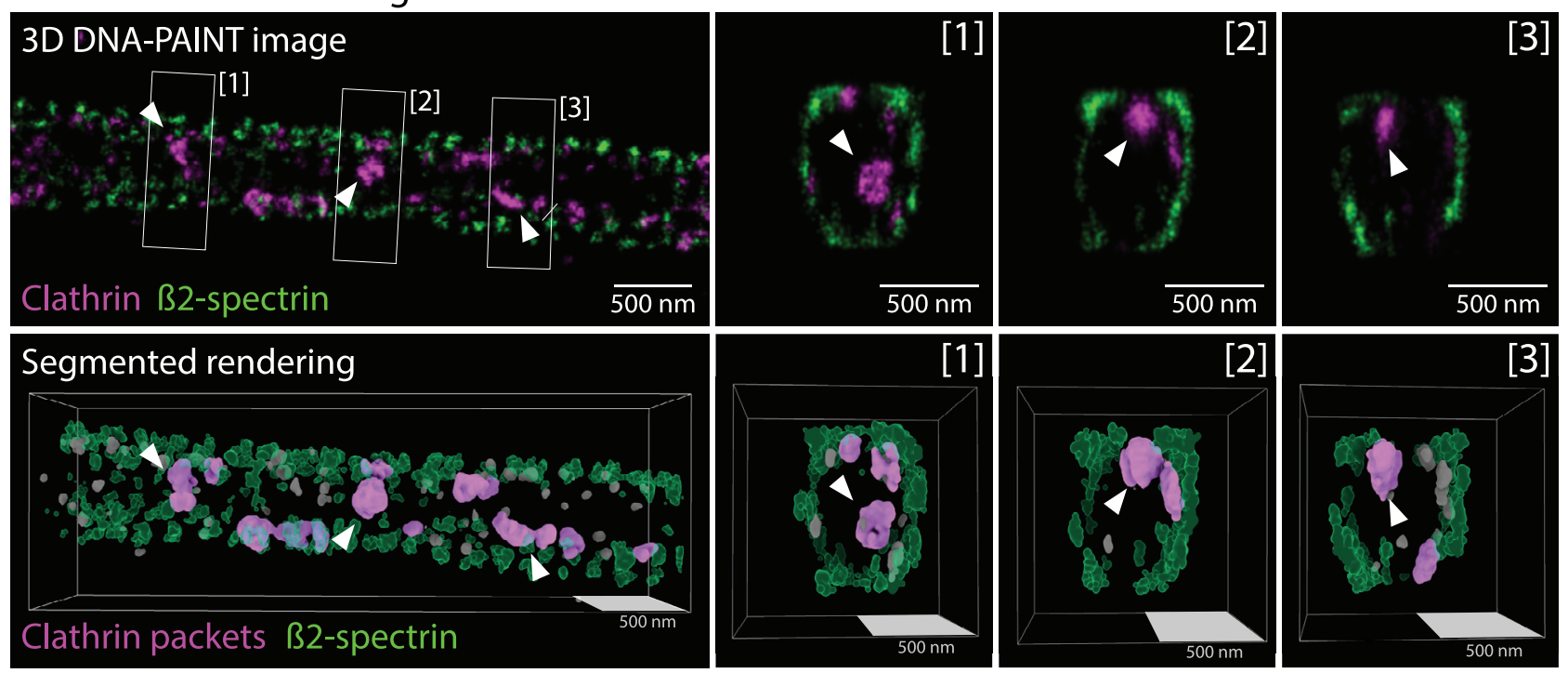

Figure 3. Ultrastructure and super-resolution imaging of axonal clathrin transport packets

(A) Strategy to visualize clathrin by APEX labeling and EM tomography.

(B) A DAB-stained neuron identified on gridded membrane (soma and axon marked by arrow and arrowhead, respectively; see also STAR methods and Figure S3). Scale bars, $30 \mu \mathrm{m}$.

(B' and B") EM of the axon above (a single plane of tomogram shown). Note clathrin-coated structures in the axon shaft (white arrowheads), with average diameters of $\sim 50 \mathrm{~nm}$ (spherical core). Representative 3D EM from three separate cultures (see STAR methods for more details). Scale bar (lower left) for B' represents $300 \mathrm{~nm}$, B" represents $300 \mathrm{~nm}$, and lower right represents $55 \mathrm{~nm}$.

(C) DNA-PAINT of endogenous clathrin in the axon shaft. Top panels: $\beta 2$-spectrin (green, to mark axonal boundaries) and clathrin heavy chain (magenta). An XY image is shown on the left, along with three Z-slices on right (1-3). Bottom panels: Segmented rendering from 3D-PAINT images (small excluded particles are in gray). Equivalent sphere diameters for the clathrin particles marked by arrowheads: 79,70 , and $70 \mathrm{~nm}$ for particles highlighted in 1,2, and 3 , respectively. Scale bars, $500 \mathrm{~nm}$.

expected, coated pits and coated vesicles were seen in soma and dendrites (Figure S2B). However, axons contained intact clathrin-coated structures that were inside the axon shaft, with no association with the axonal plasma membrane, as seen by EM tomography (Figures 3B-3B'; see also 3D view in Video S3). The diameters of these clathrin structures as determined by EM were $\sim 50 \mathrm{~nm}$ and $\sim 125 \mathrm{~nm}$ (inner spherical core and outer diameter including spokes, respectively), consistent with previous cryo-EM studies of clathrin-coated vesicles (Heymann et al., 2013).
We also used DNA-PAINT, a single-molecule-based superresolution microscopy technique (Jungmann et al., 2014), to visualize the nanoscale organization of endogenous clathrin along axons. Axonal boundaries were determined by visualizing $\beta 2$-spectrin, a component of the submembrane periodic axonal scaffold (for review, see Leterrier et al., 2017). Unlike the tedious APEX EM imaging process involving light/EM correlation of transfected axons, DNA-PAINT allowed us to visualize and characterize a large number $(>500)$ of axonal clathrin structures. From 3D multicolor DNA-PAINT images of clathrin and $32-$ 
spectrin, we generated $3 \mathrm{D}$ renderings for the segmentation and measurement of axonal clathrin particles, some of which are likely to be mobile transport packets (Figure 3C). The average equivalent diameter of clathrin heavy-chain-labeled packets was $43.3 \pm 0.5 \mathrm{~nm}$ (mean $\pm \mathrm{SEM}$; 1,294 packets), consistent with the core size of the structures seen in our EM data, and the average density was $7.6 \pm 0.5$ packets $/ \mu \mathrm{m}$ of axon (82 rendered axonal segments). Interestingly, most clathrin packets seen by super-resolution imaging were close to the $\beta 2$-spectrin sheath, suggesting associations with the submembrane actin/ spectrin scaffold (see cross-sections of axons in Figure $3 \mathrm{C}$ and Video S4). Given that the majority of axonal clathrin particles are immobile at any given time, we speculate that this may reflect anchoring of clathrin assemblies to the periodic actin/spectrin lattice.

\section{Composition of axonal clathrin transport packets}

To define the composition of axonal clathrin, we immunoprecipitated (IP) clathrin from mouse sciatic axon fractions, followed by MudPIT-MS (multidimensional protein identification technologymass spectrometry; Liao et al., 2008; Yates et al., 2009); see protocol in Figure S4A. A diverse group of cytosolic proteins were identified (Figure 4A; see Table S1 for a full list, which has also been submitted to the database PRIDE: PXD027857), and as expected, clathrin was one of the most abundant proteins (Figure 4B). We identified 115 proteins, and many were cytosolic proteins that are known to be enriched at synapses (Wilhelm et al., 2014). Network analyses showed that the axonal clathrin interactome contained many cytoskeleton-associated proteins such as actin isoforms and myosins (Figure 4A, dashed circle). Actin and clathrin have established anatomic and functional links in non-neuronal cells (for review, see Kaksonen et al., 2006). Previously, using a probe for filamentous actin (GFP tagged to the calponin homology domain of utrophin, GFP:Utr-CH), we found that axon shafts have focal actin "hotspots" every $\sim 3-4 \mu \mathrm{m}$ along their length, where actin assembles and disassembles continuously, and that these foci serve as a nidus for nucleation of actin filaments that elongate bidirectionally along the axon shaft ("actin trails"; see Ganguly et al., 2015). Interestingly, simultaneous imaging of GFP:Utr-CH and GFP:CLC showed that there was a striking colocalization of actin hotspots and stationary clathrin in axons, though the actin foci were much more short-lived, as expected (Figures $4 \mathrm{C}$ and S4B).

Network analyses also revealed that axonal clathrin was associated with proteins known to disassemble clathrin-coated pits (such as GAK, Hsc70, synaptojanin), as well as those linked to clathrin pit formation (dynamin, AP-complex); see Figure 4A. The presence of both assembly and disassembly proteins in MS datasets is intriguing, as the transported clathrin structure is clearly discrete. To test if candidate assembly/disassembly proteins from the MS dataset are indeed co-transported with clathrin, we visualized the transport of mCherry:CLC with GFP fusions labeling actin, GAK, AP180, and dynamin, using simultaneous two-color live imaging. A significant fraction of GAK was also co-transported with clathrin (Figure 4D). Like clathrin, the dynamics of GAK in dendrites was also very different from axons. As shown in the kymograph in Figure S4C, foci of GFP:GAK typically coincided with clathrin disassembly in dendrites, very different from the mixture of stationary and motile GAK particles seen in axons. Quantification showed that significant amounts of GAK, AP180, and dynamin were co-transported with clathrin (Figure 4E). In previous proteomic studies, we identified Hsc70 as a regulator of the slow axonal transport of synapsin (Ganguly et al., 2017). However, pharmacologic or genetic inhibition of Hsc70 had no effect on the axonal GFP:CLC motility (Figure S4D), suggesting that the mechanistic basis for the transport of the two slow-component cargoes, clathrin and synapsin, is different. Indeed, synapsin has an anterogradely biased diffusion-like flow in axons (Scott et al., 2011; Tang et al., 2013), while clathrin is conveyed as discrete, stable packets.

\section{Nanoscale organization of synaptic clathrin}

Clathrin is strongly enriched at presynaptic boutons and terminals, though its precise synaptic function has been controversial in recent years (Chanaday et al., 2019; Milosevic, 2018; Watanabe et al., 2013). Using light microscopy, we noticed that the presynaptic localization of clathrin was not homogeneous, like the classical vesicular or cytosolic synaptic markers synaptophysin or synapsin, but appeared as multiple puncta within a bouton. To pinpoint the spatial relationship of endogenous presynaptic clathrin to synaptic vesicle clusters, we first visualized synapses and adjacent axons at nanometer resolution in 3D, with two-color DNA-PAINT, using antibodies to clathrin and synapsin (the latter to label SV clusters; see STAR methods for details). In the cropped axon and en passant bouton shown in Figure 5A (entire image in Figure S5A), note that multiple clathrin particles (magenta) are abutting the SV cluster (green). 3D views (Figure 5B and Video S4) unequivocally show that clathrin particles are circumferentially organized around SVs and rarely seen deep within the SV cluster. Segmented rendering of the superresolution data showed that each presynapse contains $\sim 4-12$ clathrin particles (Figure $5 \mathrm{C}$ ) that were on average $\sim 43 \mathrm{~nm}$ in equivalent diameter (Figure 5D), consistent with the size of clathrin assemblies seen in axon shafts. The morphologic similarities between the axonal and presynaptic clathrin particles suggest that they are related to transport packets, and hereafter we refer to them as "synaptic clathrin packets." The large number of synaptic clathrin packets visualized by DNA-PAINT (> 1,200 packets from > 100 synapses) also allowed us to explore their correlation with SV clusters. The total volume of all clathrin packets at a given synapse correlated with volume of the SV cluster (Figure 5E), suggesting putative links between clathrin levels and SV pools. Taken together, the data suggest that motile axonal transport packets are targeted to presynapses, where they radially organize around SVs.

Though we were not able to confidently visualize the APEX-EM signal at synapses due to high background, we analyzed immuno-gold EM datasets collected by Dr. Tao-Cheng at NINDS/ $\mathrm{NIH}$ ( also see Tao-Cheng, 2020). As shown in the representative images (Figure 5F), immuno-gold particles were mostly seen around-and not within-SV clusters, and some gold particles were associated with vesicular structures resembling endosomes and cisternae (asterisk in Figure 5F, subpanel 2). Coated vesicles are rarely seen at synapses, as reported by previous EM studies (Heuser and Reese, 1973; Kononenko et al., 2014; Petralia et al., 2003; Tao-Cheng, 2020). Taken together, the super- 


\section{A Protein interactome of axonal clathrin}

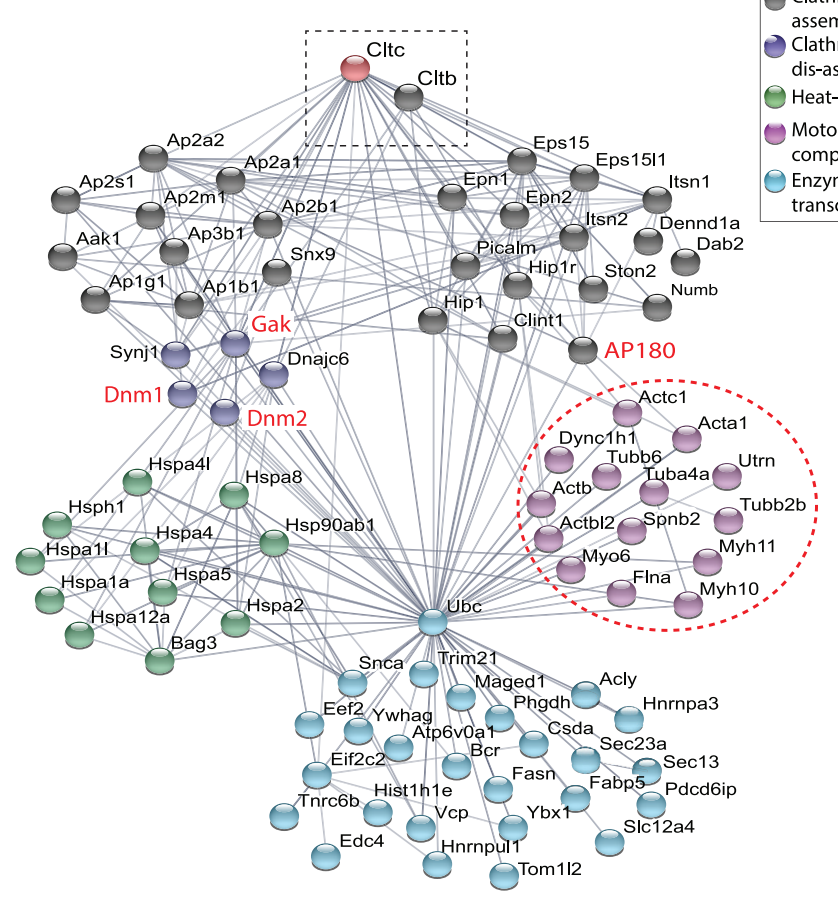

Clathrin heavy chain Clathrin coated pitassembly proteins Clathrin coated pits-assembly proteins t-shock proteins otors and cytoskeleta components Enzymes and transcription factors
B MUDPIT/MS and Co-IP (WB)

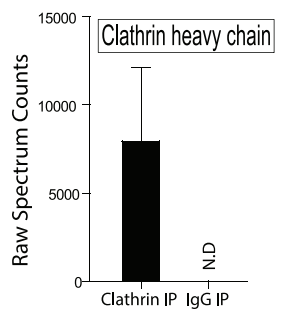

C Colocalization of clathrin and actin

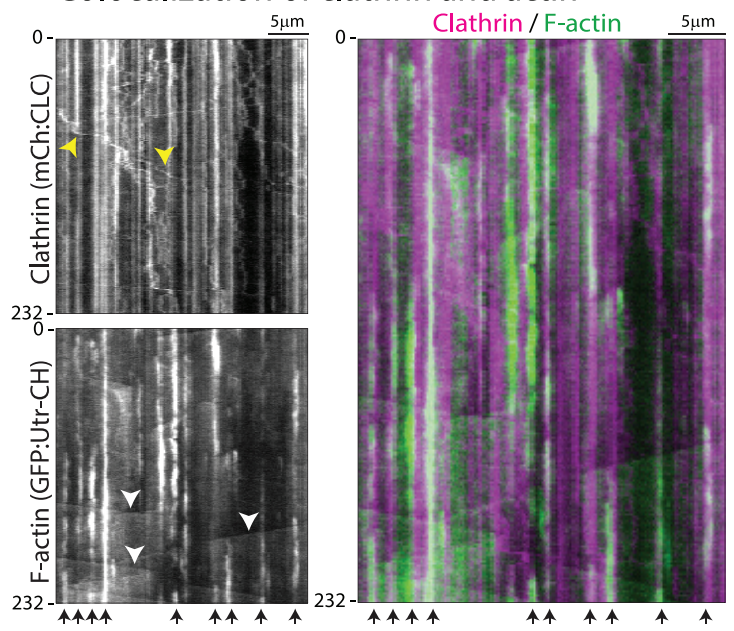

D
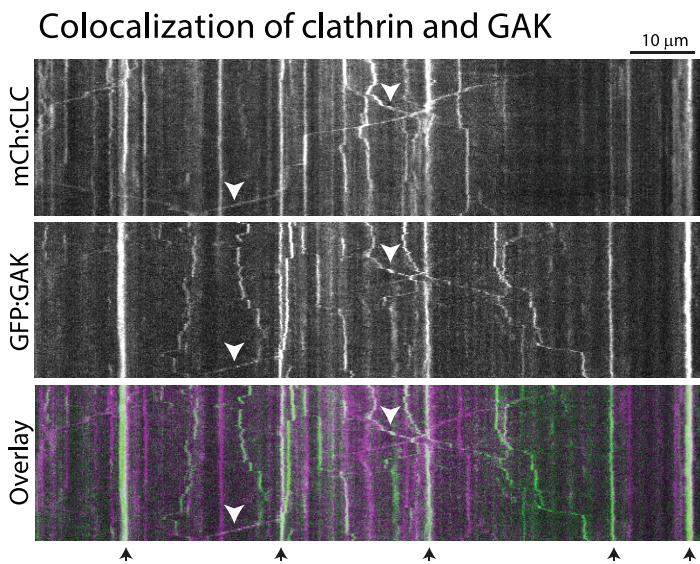

\section{Colocalization of clathrin with $\mathrm{M} / \mathrm{S}$ candidates}

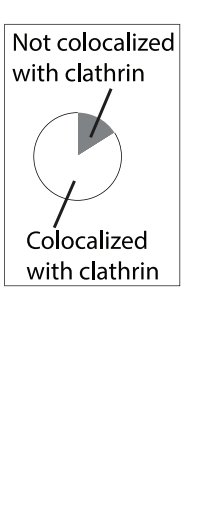

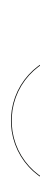

GAK
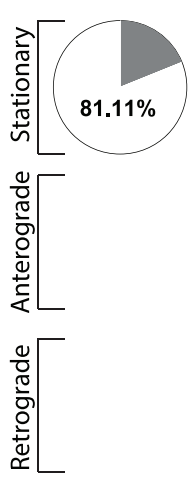

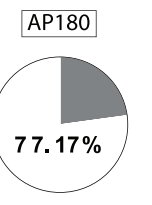

Dynamin
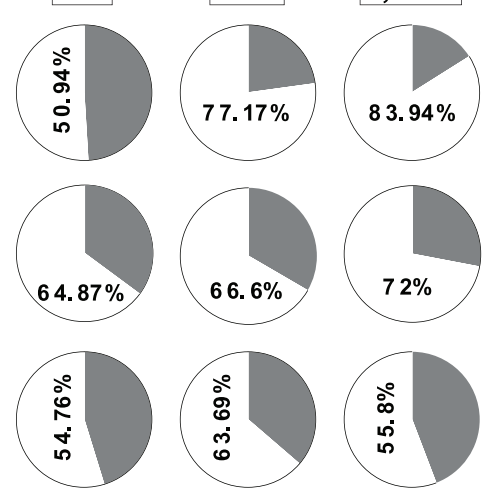

Figure 4. The axonal clathrin interactome

(A) An interactome of clathrin-associated proteins (clathrin marked by dashed rectangle), grouped into functional clusters. The "actin hub" (dashed circle) was of particular interest as previous studies have established mechanistic links between clathrin and actin (see also Results).

(B) High clathrin spectrum counts in MS data, confirmed by western blots showing clathrin in IP fractions.

(C) Kymographs from axons of neurons co-transfected with mCherry:CLC and a GFP-tagged probe for F-actin (GFP:Utr-CH). Note extensive colocalization of stationary clathrin and actin (arrowheads at the bottom, merged kymograph on right). Moving clathrin particles and "actin trails" are marked on the kymographs by yellow and white arrowheads, respectively. Time in seconds is marked on the left of the kymographs, and scale bar on upper right. Scale bars, $5 \mu \mathrm{m}$.

(D) Kymographs from axons of neurons co-transfected with mCherry:CLC and GFP:GAK. Note colocalization of stationary particles (arrowheads at the bottom of kymographs), and co-transported clathrin and GAK particles (arrowheads). Time in seconds is marked on the left of the kymographs, and scale bar on upper right. Scale bars, $10 \mu \mathrm{m}$.

(E) Quantification of kinetic data from simultaneous imaging of of clathrin with candidates from MS data (GAK, AP180, and dynamin). Note that a substantial amount of clathrin particles were co-transported with GAK, AP180, and dynamin ( 140-340 particles were analyzed from 12-19 neurons; data from 2-3 separate cultures). 


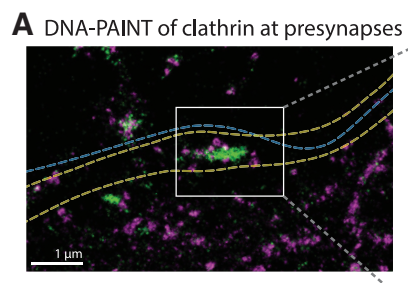

C Number of clathrin particles
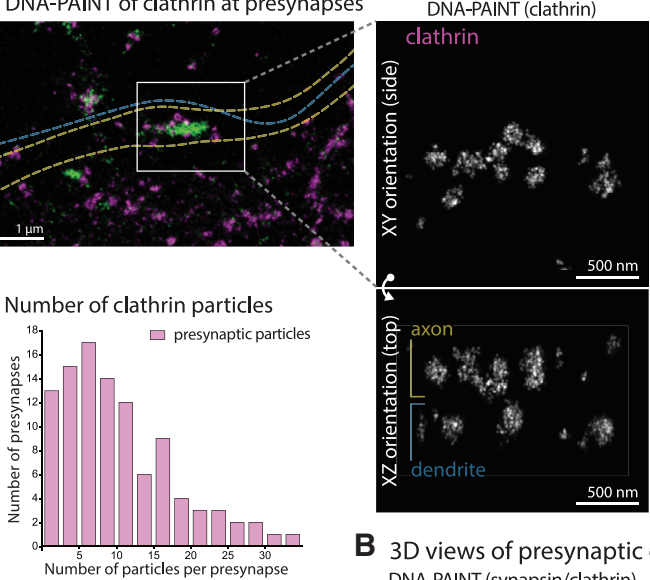

DNA-PAINT (synapsin/clathrin)
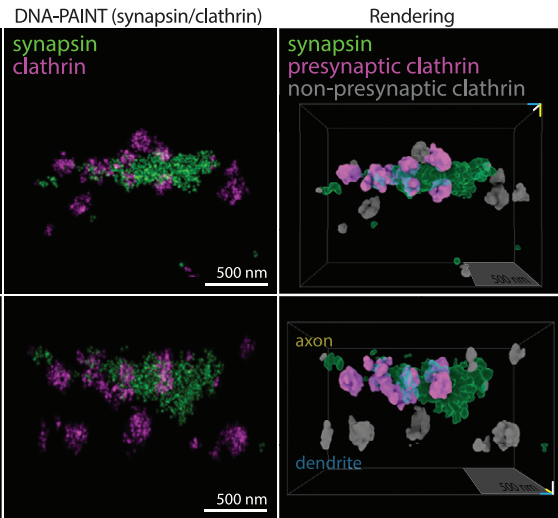

B 3D views of presynaptic clathrin particles

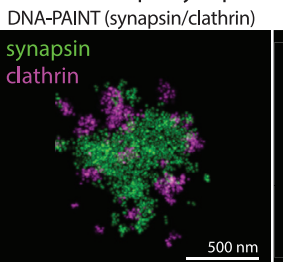

$X Y$ rendering

D Diameter of clathrin particles

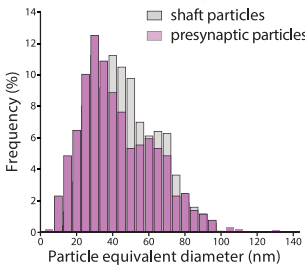

E Presynaptic volumes correlation
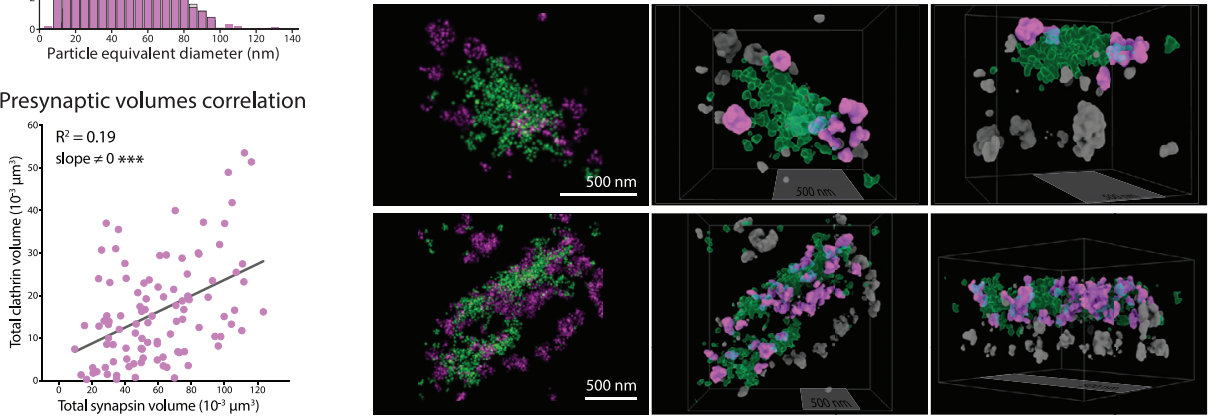

F Immunogold EM of clathrin

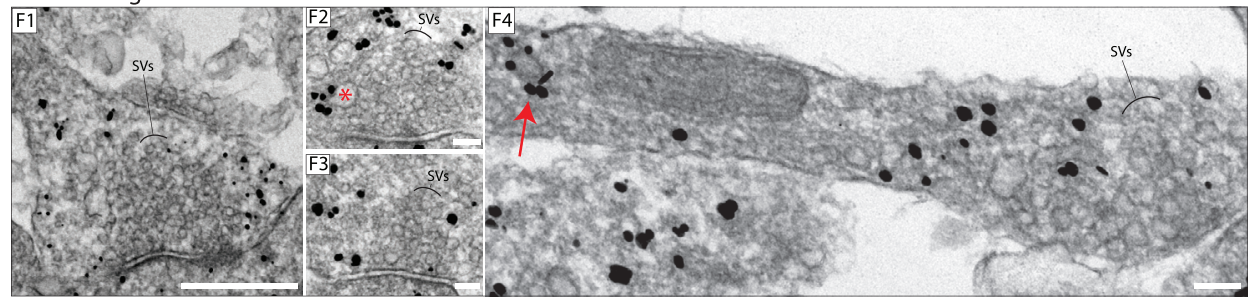

Figure 5. The nanostructure of clathrin at the presynapses

(A) Left: DNA-PAINT of endogenous clathrin (magenta) and synapsin (green, SV marker) at an en passant bouton. Outline of a dendrite (blue dashed line) and axon (yellow dashed line) are shown (see full image in Figure S5A); scale bars, $1 \mu \mathrm{m}$. Right: 3D views of rectangular inset with XY and XZ (rotated) views, along with 3D rendering and segmentation (extreme right panels). The rendered view shows presynaptic clathrin in magenta, non-presynaptic clathrin in gray, and synapsin in green. Note clathrin particles abutting the presynaptic SV cluster. See also Video S4. Scale bars, $500 \mathrm{~nm}$.

(B) 3D views of more presynapses showing DNA-PAINT image and segmented rendering (see also Video S4). Scale bars, $500 \mathrm{~nm}$.

(C) Histogram of segmented presynaptic clathrin particles per presynapse (1,261 clathrin particles from 102 synapses analyzed).

(D) Overlaid histograms showing frequency distribution of presynaptic clathrin particle diameters (1,261 particles from 3 independent experiments), compared to clathrin particle diameters in axon shaft (685 particles from 3 independent experiments).

(E) Total clathrin volume (cumulative of all clathrin particles) at a given bouton correlated with the total volume of synapsin

(F) Immuno-gold EM of clathrin ( silver enhancement of nanogold particles). F1-F4: Note most particles are located at the periphery of SV clusters, and some are associated with cisternae that may be endosomes (red asterisk in F2 for example). F4 shows a segment of an axon with presynapse, and red arrow marks a coated structure resembling an axonal transport packet. Note that the labeling is specific for clathrin; see also Figure S5B. Scale bar (lower right) for F1 is $500 \mathrm{~nm}$ and for F2, F3, and F4 is $100 \mathrm{~nm}$. F1-F4: photo courtesy from Jung-Hwa Tao-Cheng (NINDS/NIH). 
resolution and immuno-EM data, along with previous studies, indicate that endogenous synaptic clathrin localizes at the periphery of SV clusters, and the majority of synaptic clathrin is not in the form of coated vesicles.

\section{Clathrin packets are captured and immobilized at synapses}

To explore the dynamics of synaptic clathrin, we first visualized GFP:CLC at synapses with relatively high temporal resolution (5 frames/s). Motile clathrin particles rapidly moved between boutons (average velocity $0.54 \mu \mathrm{m} / \mathrm{s}$ ) and were often trapped at synapses (kymographs in Figures 6A and S6A). A "pass/trap analysis" (see Figure S6B and STAR methods) indicated that $\sim 60 \%$ of the moving particles were retained at boutons for at least a minute (129 particles analyzed from 19 neurons, 3 separate cultures). Within a bouton, clathrin particles moved back and forth, seemingly constrained by the synaptic boundary, though they could exit (Figure 6A'). Single synaptic GFP:CLC particles could be tracked for only a few seconds, suggesting instability (see photoconversion data later). Next, we used FRAP to bleach GFP:CLC in a single bouton and visualized fluorescence recovery over time, reflecting the entry of unbleached fluorescent molecules from adjacent boutons/axons into the bleached zone. As shown in Figure 6B, there was a slow recovery of fluorescence over several minutes, and particles were frequently seen to enter the bleached bouton (best seen in Video S5). Interestingly, FRAP of GFP:CLC fluorescence at boutons was qualitatively very different from GFP:synapsin. While recovery of clathrin was largely due to entry of discrete fluorescent structures-and was somewhat stochastic-synapsin FRAP was gradual and diffuse (Figures 6C and S6C). Also, clathrin mobility in presynaptic boutons decreased over time in our cultures (Figure S6D), likely due to synaptic maturation, reminiscent of the on/off behavior of GFP:CLC in dendrites, which also decreases with time (Blanpied et al., 2002).

Fluorescence recovery of a freely diffusing molecule is expected to be complete (see FRAP of soluble, untagged GFP in boutons, Figure $6 \mathrm{D}$ inset), and incomplete recovery reflects a bound, immobile pool that was unable to enter the bleached zone (Lippincott-Schwartz et al., 2003). Though FRAP of both clathrin and synapsin was incomplete, clathrin had a much larger immobile fraction (Figures 6D, 6E, and S6E). A previous study in C. elegans found that endophilin, a soluble protein involved in endocytosis, showed diffusion-like mobility between synapses that was blocked when SV recycling was perturbed (Bai et al., 2010); suggesting that the trafficking endophilin pool was generated locally after SV recycling. Though the diffusion-like kinetics of endophilin is very different from the particle movement of clathrin, it is possible that the inter-synaptic movement of clathrin is also dependent on endocytosis, and that the clathrin packets are locally generated at the synapse. To test this, we performed clathrin FRAP experiments before and after adding Dynasore. Dynasore did not attenuate FRAP of clathrin, and unexpectedly, there was an increase in clathrin motility (Figure 6F). One possibility is that blocking endocytosis might decrease the usage of clathrin packets at synapses, allowing more packets to shuttle between synapses (perhaps also increasing their synaptic capture). Indeed, after Dynasore treatment, there was an increase in the number of clathrin packets exchanging between boutons, i.e., exported from one synapse and captured at another (Figure 6G). Collectively, the data suggest that the "superpool clathrin packets" are related to trafficking and not generated at synapses following endocytosis. The increase in the synaptic capture after Dynasore also suggests that synaptic targeting of clathrin may be independent of its function at the synapse.

The morphology and dynamics of synaptic clathrin puncta suggest that axonal transport and subsequent presynaptic "trapping" of a discrete number of transport packets may be the basis of clathrin enrichment at boutons. To further explore morphologic similarities between the transported clathrin and the clathrin localized at synapses, we asked if the total fluorescence of clathrin at individual boutons was an aggregate of integer multiples of axonal puncta/transport packets. Overlaid histogram distributions of GFP:CLC fluorescence intensities in axons (green) and presynapses (red) are shown in Figure $6 \mathrm{H}$ (left). Note that while GFP:CLC axonal intensities occupy a single peak, synaptic GFP:CLC intensities are distributed as multiple peaks of higher intensities. Figure $6 \mathrm{H}$ (middle) shows predicted fluorescence peaks of synaptic clathrin if they were integer multiples of the axonal intensity peak (assumed as " $x 1$ "). From this analysis, single boutons are predicted to contain $\sim 4-8+$ clathrin packets (Figure $6 \mathrm{H}$, right). A similar distribution of axonal and synaptic fluorescence was also seen for endogenous clathrin (Figure S6F). Collectively, the data are consistent with a model in which synaptic enrichment of clathrin is largely due to clathrin packets shipped by slow axonal transport.

\section{Dynamics of synaptic clathrin packets}

Next, we used photoconvertible clathrin to determine the fate of synaptic clathrin. Neurons were transfected with Dendra2:CLC (green to red photoconversion), and synaptic clathrin was identified by constitutive green fluorescence (see STAR methods for details). Dendra2:CLC in a single bouton was photoconverted to red, and both green (stable) and red (photoconverted) fluorescence was monitored over time. Photoconverted clathrin particles moved out of the synapse into the flanking axons and were often trapped at adjacent boutons (Figure 7A; see also Video S6). Pass/trap analysis showed that $\sim 67 \%$ of moving particles were trapped (see Figure S6B and STAR methods for details; 46 particles analyzed from 14 neurons; 3 separate cultures). The overall dispersion of clathrin from photoconverted boutons, determined by decay of red fluorescence over time, is shown in Figure 7B. Previous studies have described a mobile vesicle superpool spanning multiple synaptic boutons and flanking axons, composed of vesicles that are available to synapses during stimulation (Darcy et al., 2006; Krueger et al., 2003; Staras et al., 2010), and pharmacologic experiments suggest that the SV superpool is actin dependent (Darcy et al., 2006; Ratnayaka et al., 2011). Though the kinetics of the clathrin particles in and around synapses resemble the vesicle superpool, interestingly, the peri-synaptic motility of photoconverted Dendra2:CLC particles was microtubule-and not actin-dependent (Figures $7 \mathrm{C}$ and 7D).

To explore the dynamics of individual synaptic packets, we photoconverted a single Dendra-2:CLC packet within a bouton and visualized both stable (green) and photoconverted (red) 
A GFP:CLC trafficking at synapses

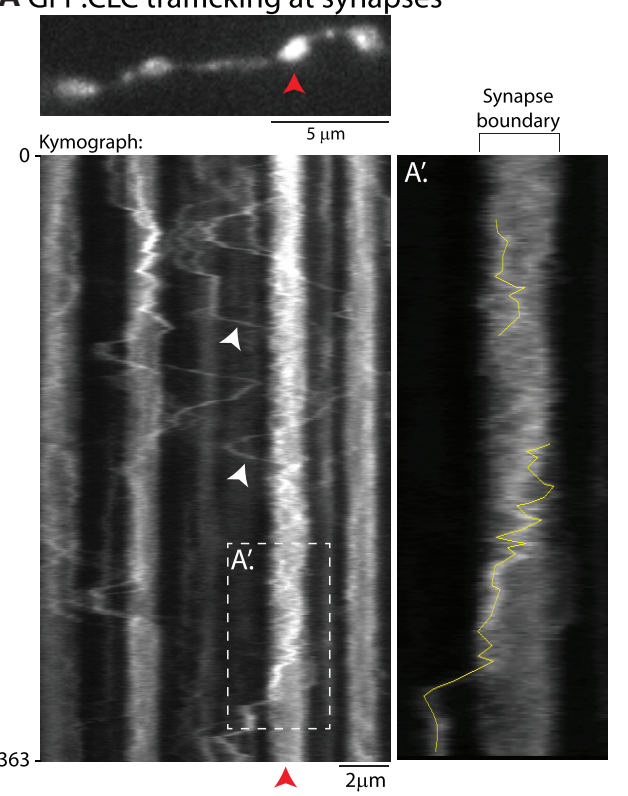

B GFP:CLC photobleaching at synapses
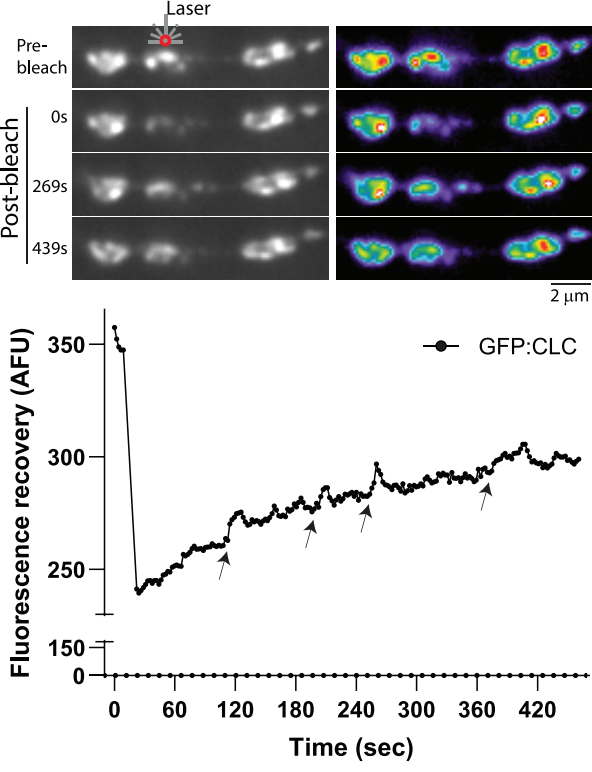

$E$

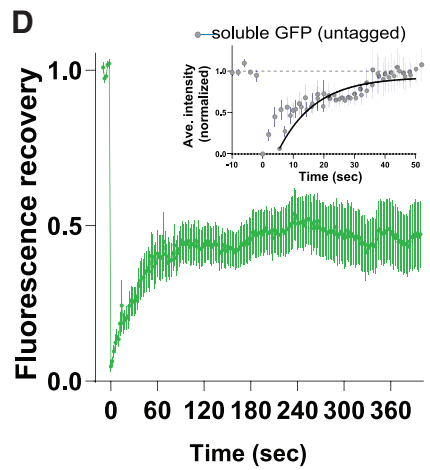

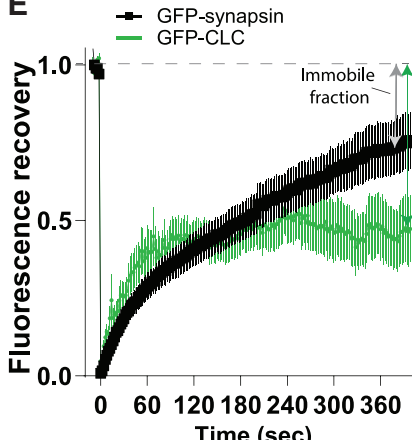

F $\quad-$ GFP:CLC Cont $\left(\mathrm{t}_{12}=33 \mathrm{~s}\right)$

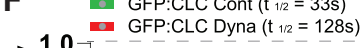

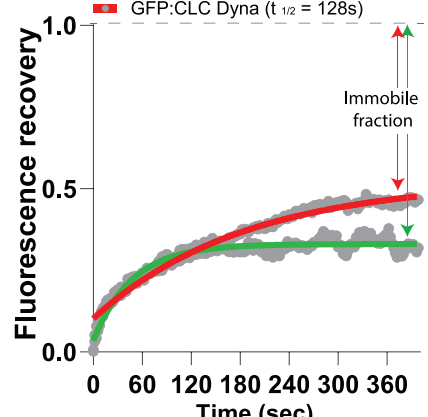

C

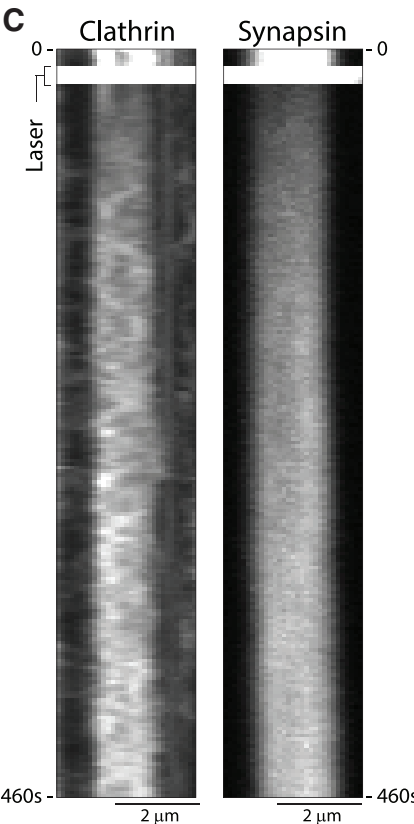

G OGFP:CLC Cont 25 O GFP:CLC Dyna

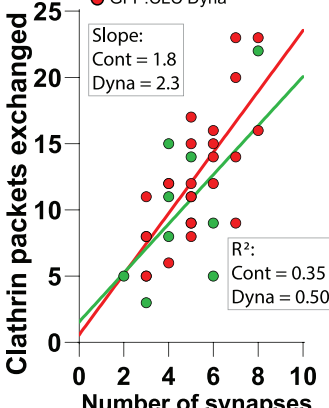

H Axonal and synaptic clathrin intensities
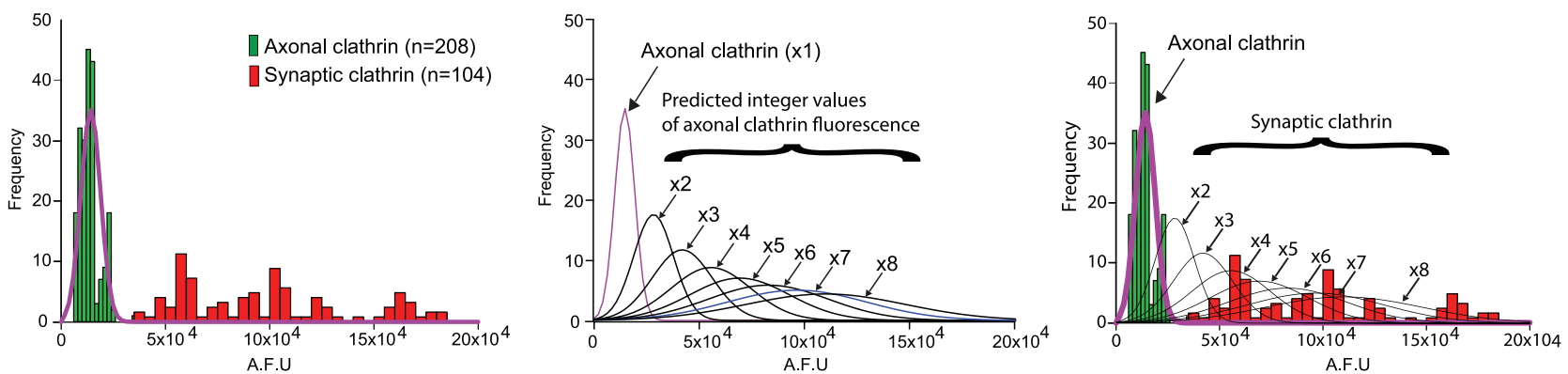

Figure 6. Transported clathrin packets are captured and immobilized at presynapses

(A) Rapid imaging of GFP:CLC around boutons, with first frame on top and kymograph at bottom. Note that clathrin particles are rapidly transported between boutons and often localize to synapses (white arrowheads, targeting to synapse marked by red arrowhead). (A') is a zoom of the boxed region in kymograph showing trajectories of two particles. Scale bar for upper left, $5 \mu \mathrm{m}$; lower left, $2 \mu \mathrm{m}$.

(B) GFP:CLC was bleached in a single bouton and fluorescence recovery was monitored over time, as shown in the time-series grayscale and pseudocolor heatmap images. Note gradual recovery of fluorescence over time, also evident in the FRAP curve below (small arrowheads point to transient peaks due to particle entry into bleached bouton; see also Video S5). Scale bars, $2 \mu \mathrm{m}$.

(C) Bouton kymographs from FRAP of GFP:CLC and GFP:synapsin. Note that while fluorescence recovery of clathrin is particulate, the rise of synapsin fluorescence is diffusive. Scale bars, $2 \mu \mathrm{m}$.

(D) Bouton FRAP recovery of GFP:CLC was incomplete, suggesting an immobile pool of clathrin (FRAP of soluble, untagged GFP in boutons recovered completely, inset). For GFP:CLC, 12 neurons were analyzed from 3 separate cultures. 
fluorescence by live imaging. Given the stability of the axonal transport packets, we expected that the fluorescence would be stable in the photoconverted synaptic packet as well. Surprisingly, however, the photoconverted synaptic packet lost fluorescence over tens of seconds, while the other clathrin packets within the same bouton-identified by the stable green fluorescence-gained fluorescence over the same time frame (Figure 7E and Video S7; more examples in Figures S7A and S7A'). Clathrin exchange between synaptic packets was not seen in boutons from neurons fixed with paraformaldehyde (Figure S7B). To quantify the aggregate data from these experiments, we compared the change of fluorescence in the photoconverted packet in a bouton to that of the non-converted ("dark") packets within the same bouton (see basic principle in Figure 7F schematic). If there was exchange, fluorescence would be lost from the photoconverted packet, and there would be a gain of fluorescence in the "dark" regions. As shown in in the graphs in Figure 7F, fluorescence was lost from photoconverted packets in living neurons (left red bar) and gained in the non-photoconverted dark regions (left black bar). However, in fixed boutons, fluorescence was not lost from the photoconverted packet (right red bar, Figure $7 \mathrm{~F}$ ), and there was little change of fluorescence in the non-photoconverted dark regions of the synapse (right black bar, Figure 7F). Occasionally in axons, there was a mechanical dispersion of the axonal fluorescence due to other moving axonal packets that happened to cross the field of view (see Figure S7C for an example). A sideby-side comparison of synaptic and axonal Dendra2:CLC photoactivation is shown in Figure S7D. Collectively, the data suggest that though synaptic clathrin packets are derived from axonal packets, the packets become much more dynamic upon arriving at synapses.

\section{Physiologic role of clathrin packets}

Do the clathrin transport/synaptic packets have a physiologic role at synapses? To address this, we designed a system to sequester clathrin packets in the neuronal soma-depleting them from synapses-and then examined SV recycling in the clathrin-depleted boutons using FM4-64, a red styryl dye that is selectively endocytosed into rapidly recycling SVs and has been widely used to evaluate synaptic function (Gaffield and Betz, 2006). Though previous studies have used shRNAs to deplete clathrin over several days, clathrin is required for Golgi formation (Radulescu et al., 2007) and essential for survival (Bazinet et al., 1993). Thus, we sought a more acute knockdown of clathrin, and also a method that visibly depleted the packets from synapses. Toward this, we used the FKBP-Rapalog-FRB heterodimerization system, in which proteins fused to FKBP or FRB dimerize upon adding Rapalog (Belshaw et al., 1996; Bentley et al., 2015; Kapitein et al., 2010; Robinson et al., 2010). We tagged FRB to GFP:CLC (FRB-GFP:CLC), and FKBP to the dynein-binding fragment of BicD2 (BicD2FKBP \pm Tdtomato tag), a dynein cargo adaptor that moves cargoes toward the minus end of microtubules (Dienstbier and Li, 2009).

In our system, Rapalog would induce dimerization of GFP:CLC-labeled clathrin packets and BicD2, transporting them toward the minus end of axonal microtubules and into the neuronal soma, thus depleting clathrin packets from boutons (see schematic in Figure 8A). Figure 8B shows examples of neurons transfected with FRB-GFP:CLC and Tdt:BicD2-FKBP, with or without Rapalog. Note sequestration of GFP:CLC in neuronal cell bodies and its depletion from synapses after incubation with Rapalog (Figure 8C, quantified in Figure 8D). Importantly, almost all of the transfected FRB-GFP:CLC co-assembles with endogenous axonal clathrin (Figures S8A and S8B); thus sequestering FRB-GFP:CLC leads to a depletion of all synaptic clathrin in these experiments, including endogenous clathrin (see Figure $8 \mathrm{E}$ ). Neurons appeared healthy with normal axonal and dendritic arborizations after FKBP/FRB/Rapalog-mediated clathrin sequestration for up to $10 \mathrm{~h}$, and a live/dead assay (Liljebäck et al., 2019) showed no toxicity (Figure S8C).

Next we evaluated SV recycling in the clathrin-depleted boutons using FM-uptake protocols that specifically label the recycling pool (Cousin et al., 2018; see Figure 8F and STAR methods; for discussion of various synaptic pools, see Denker and Rizzoli, 2010). Incubation with Dynasore led to $\sim 75 \%$ decrease in FM uptake (Figure S8D), and as shown in Figure 8G, there was also a significant reduction in FM-dye uptake in the Rapalogtreated neurons (quantified in Figure $8 \mathrm{H}$, similar changes were seen at room temperature as well as $37^{\circ} \mathrm{C}$; see Figure S8E). Interestingly, though Rapalog treatment for $10 \mathrm{~h}$ led to a greater attenuation of synaptic clathrin when compared to the $5 \mathrm{~h}$ Rapalog treatment (see Figures 8D, 8E, and 8G, left graph), the extent of FM attenuation was similar at both $5 \mathrm{~h}$ and $10 \mathrm{~h}$ time points, suggesting that there may be a finite pool of synaptic clathrin that participates in function, and the remainderacts as a reservoir to help maintain homeostatic protein levels at this locale. Taken together, the data suggest that clathrin packets at the synapse are involved in maintaining SV recycling.

(E) Comparison of GFP:CLC and GFP:synapsin bouton FRAP. Note that clathrin has a larger immobile pool than synapsin (12-14 neurons from 2 or 3 separate cultures were analyzed).

(F) Blocking endocytosis with Dynasore attenuated the immobile fraction of GFP:CLC in bouton-FRAP experiments (i.e., increased mobility of clathrin packets), indicating that FRAP recovery was not due to clathrin on newly generated endosomes at adjacent synapses (11-15 neurons from 3 separate cultures for each condition). For each experiment, the same coverslip was evaluated before/after Dynasore to minimize variability.

(G) More GFP:CLC particles exchanged between synapses after Dynasore treatment (moved from one synapse into another and stayed there for at least a minute). 200-400 particles moving between 100 synapses (16-21 neurons per condition) were analyzed.

$(H)$ Integers of axonal clathrin packets localize to synapses. Left: Histogram showing distribution of GFP:CLC intensities in axons and synapses. Note that while axonal clathrin intensities are tightly clustered in a single peak (green), synaptic intensities are distributed as multiple peaks of fluorescence (red). Middle: Mean fluorescence of axonal clathrin was considered as 1x, and hypothetical integer multiple curves were generated (fitted curves shown). Right: Axonal and synaptic GFP:CLC fluorescence data were overlaid with the curves shown in the middle panel. Note that data predict that the synaptic clathrin fluorescence is composed of 4-8+ integer multiples of axonal fluorescence (100-200 particles were analyzed from 3 separate cultures).

Key for kymographs: elapsed time in seconds on left or right and scale bar on lower right (laser illumination also marked). 
A Photoconversion of synaptic clathrin (total bouton)

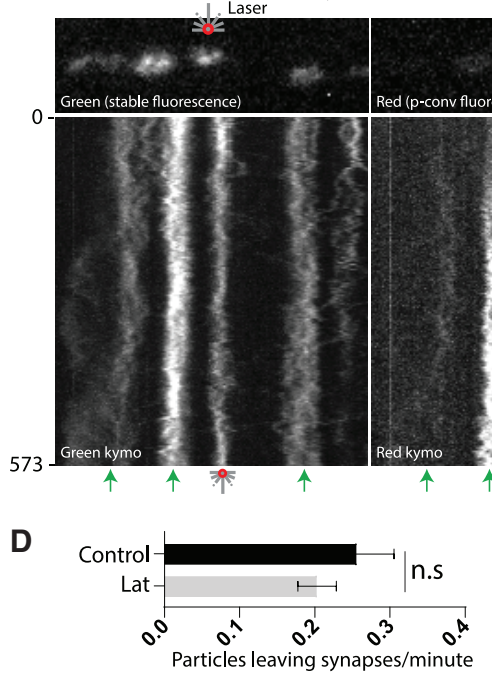

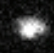

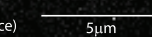

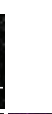

Stable clathrin
Photoactivated clathrin d Laser $5 \mu \mathrm{m}$

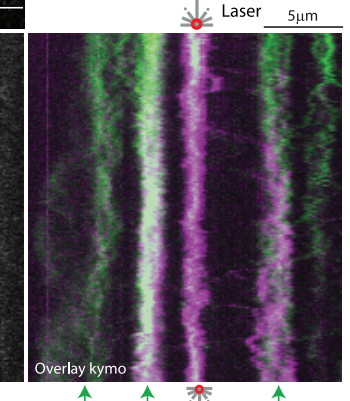

ControlParticles leaving synapses/minute

E Photoconversion of synaptic clathrin packets

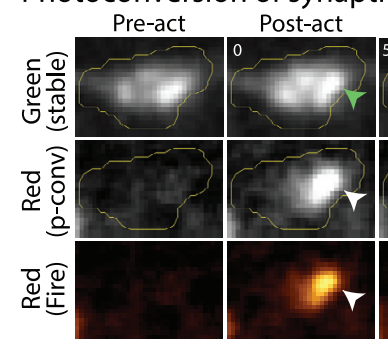

F

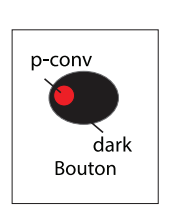

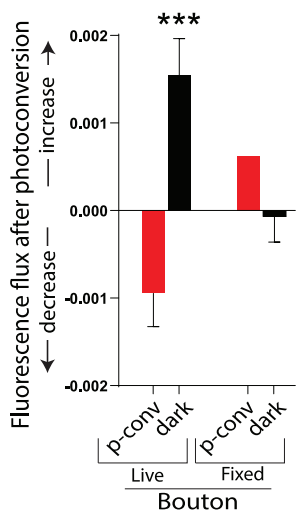

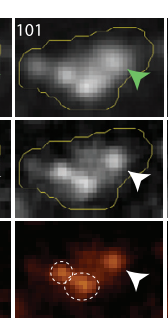

G Photoconversion of clathrin in axon shaft

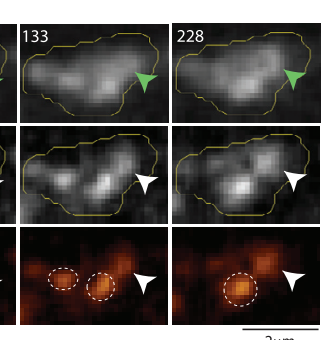

B Clathrin dispersion from synapses

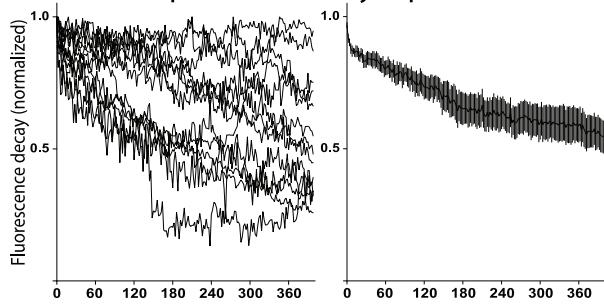

C Clathrin motility after nocodazole

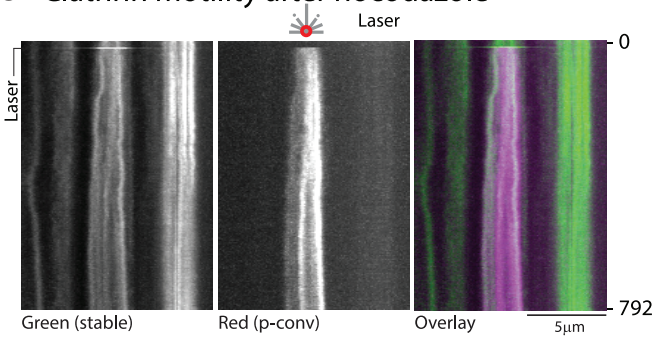

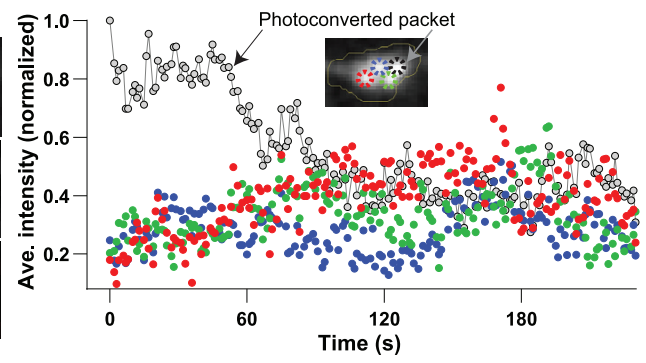
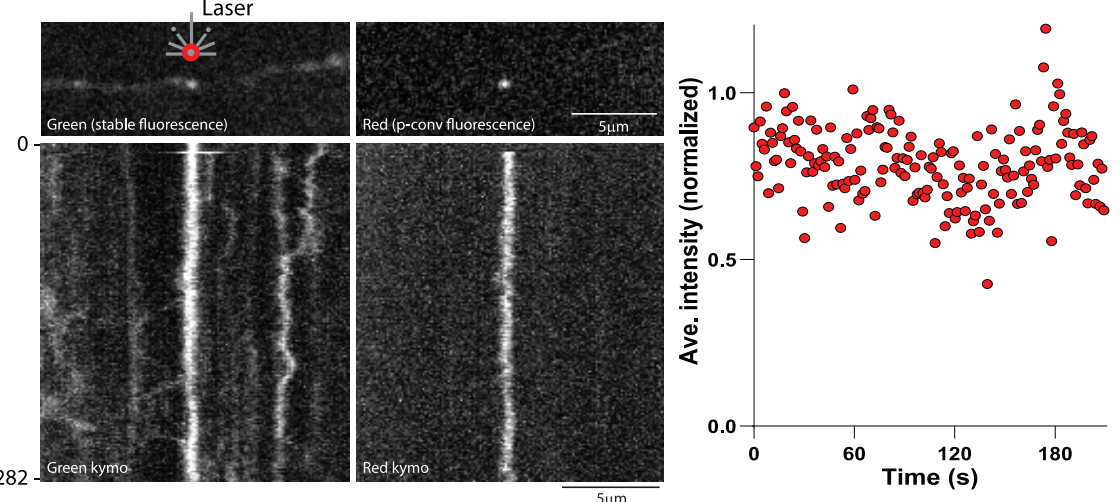

Figure 7. Dynamics of synaptic clathrin packets

(A) First post-photoconverted frames (above) and kymographs (below) from photoconversion of Dendra2:CLC at a single bouton. Photoconverted bouton is marked by red laser symbol and non-photoconverted boutons by small green arrows at bottom. Note that over time, fluorescent clathrin particles leave the single photoactivated bouton and are immobilized at adjacent boutons (white arrowheads in middle kymograph).

(B) Decay of photoconverted Dendra2:CLC red fluorescence from single boutons (raw and ensemble data; 13 neurons from 2 separate cultures).

(C) Nocodazole (10 $\mathrm{\mu g} / \mathrm{mL}$ for $40 \mathrm{~min}$ ) attenuated export of synaptic clathrin particles in single-bouton photoconversion experiments, whereas latrunculin (100 $\mathrm{nM}$ for $15 \mathrm{~min}$ ) had no significant effect.

(D) Quantificaton of data from (C)-15-20 neurons from 2 or 3 separate cultures analyzed for each control- and drug-treated group.

(E) Left: Dendra2:CLC was photoconverted in a single synaptic clathrin packet (arrowhead, elapsed time in seconds on top left). Note that after photoconversion (middle and bottom panels), the photoconverted packet lost fluorescence, while there was an increase of fluorescence in adjacent packets (identified by stable green fluorescence, some marked by dashed circles in bottom panel). Right: Quantification of intensities in individual packets (normalized to highest postconversion intensity). Colors in graph correspond to the colors of circles marking packets in inset image. Black dashed circle marks the green clathrin packet that was photoconverted to red. Note reciprocal fluctuations in photoconverted and non-photoconverted packets. Scale bars, $2 \mu \mathrm{m}$. 


\section{DISCUSSION}

Our main findings are: (1) clathrin and related proteins are conveyed in axons as stable coated structures (transport packets), moving intermittently on microtubules with an overall slow anterograde bias; (2) at synapses, most clathrin assemblies (synaptic packets) localize to the periphery of SV clusters, and despite morphologic similarities with transport packets, most synaptic packets lack a conventional coat; (3) mobile clathrin packets shuttle between synapses in a microtubule-dependent superpool, and a significant fraction ( 60\%) of the moving clathrin is captured at boutons; (4) though axonal clathrin packets are stable, single synaptic clathrin packets can exchange with other neighboring packets within a bouton; and (5) depletion of synaptic clathrin packets impairs SV recycling.

\section{A model for trafficking and presynaptic targeting of clathrin}

The most straightforward model emerging from our data is that clathrin and related proteins are synthesized in the neuronal soma, where they are packaged into stable coated structures that are dedicated for transport-with no role in endocytosis. Transport packets move intermittently on microtubules and are conveyed to synapses with an overall anterograde bias in slow axonal transport. Upon reaching the synapse, the conventional coat on the packets is disrupted-perhaps due to the high concentration of uncoating proteins like $\mathrm{Hsc70}$ at this locale (Ganguly et al., 2017)-allowing clathrin to dynamically exchange with other local packets. Thus, at synapses, clathrin molecules would either be associated with a synaptic packet or exchange between local packets. This metastable state would provide a labile local pool of clathrin that could be rapidly recruited for synaptic function, as needed. In this hypothetical model, clathrin would undergo a seamless transition from transport to function upon reaching the synapse, simply by dynamically shedding the coat on the transport carrier.

Despite the dynamic exchange, our experiments strongly suggest that the synaptic clathrin is not freely diffusible as commonly believed. For instance, in photoconversion experiments (Figure $7 \mathrm{~A}$ ), we only saw discrete clathrin particles leave boutons, and incubation with nocodazole-which stalled the mobility of the synaptic clathrin packets-did not lead to a loss of clathrin from the stalled structures over time (Figure 7C), which would be predicted if this clathrin was freely diffusible. However, the precise structures on which the synaptic clathrin localizes are still unclear, though immuno-EM data suggest that some of the clathrin may be on cisternae/endosomes (Figure 5F). Our MS data and two-color live imaging also suggest that many proteins related to endocytosis are co-transported with clathrin, so once the transport packets reach the synapse, perhaps they too would be available for performing their functions. Thus, conceptually, our model may have broad consequences for neuronal form and function.

Synapses have integer multiples of clathrin transport packets (Figure 6G), which is also consistent with our working model wherein clathrin trafficking and targeting are interconnected events. However, EM suggests that while axonal clathrin packets are coated, most of the synaptic clathrin lacks a conventional coat. Previous studies have shown that clathrin-coated vesicles are infrequent in resting hippocampal synapses-for example, in a recent detailed study, only $\sim 5-10$ coated vesicles were seen per 100 presynaptic profiles (Tao-Cheng, 2020)-but it is clear that clathrin is abundant at synapses, obvious by routine immunostaining. Our live-imaging, super-resolution, and EM data provide some clarity on this issue, showing that most of the synaptic clathrin (1) is organized into discrete structures, and not as free monomers, and (2) does not resemble conventional clathrincoated vesicles. Another prediction of our model is that during transport, the stable clathrin on axonal transport packets would not be functionally available, which might be a way of preventing unwanted endocytosis along the axon shaft.

\section{Clathrin transport packets: a carrier for slow axonal transport}

Though the phenomenon of cytosolic slow transport is established, the nature of transported cargoes has been puzzling. In previous imaging studies of other slow-component cargoes (like synapsin and CamKII), we saw transient mobile structures-lasting for a few seconds at best-that appeared to contribute to the overall anterograde movement (Roy, 2014; Scott et al., 2011), but the exact cargo structure remained unclear. The discrete nature of axonal clathrin enabled us to examine the cargo structure at a nanoscale level by 3D EM and super-resolution imaging, and clathrin is probably the first cytosolic cargo to be characterized in detail. Based on the overlapping of radiolabeled "peaks" in axons, previous studies hypothesized that common mechanisms were involved in slow transport ( for review, see Roy, 2014, 2020). However, the synaptic dynamics of two slow transport cargoes, clathrin and synap$\mathrm{sin}$, is very different (Figure 6C), and an emerging theme in slow transport is the diversity in the nature and mechanisms of movement (Roy, 2020).

The structure of an axonal transport packet resembles clathrin-coated vesicles. However, coated vesicles are thought to be transient intermediates that deliver endocytosed vesicles and their contents either to Golgi/endosomes or back to the plasma membrane (for review, see Paraan et al., 2020), while the transport packets are long lasting structures that are

(F) Quantification of fluorescence change after single-packet photoconversion in boutons. Schematic on left shows general principle of quantifying photoconverted and non-photoconverted (dark) regions; see STAR methods for details. Note loss of intensity from photoconverted packet within bouton (red bar, left), with a corresponding gain in adjacent non-converted regions (black bar, left). Similar reciprocal changes in fluorescence were not seen after fixing the neurons, though the photoactivation still occurred as expected (red/black middle bars; 6 neurons from 1 culture for fixed boutons and 10 cells from 2 separate cultures for live boutons).

(G) Photoconversion of single Dendra2:CLC clathrin packet in axon. Note no change in fluorescence, quantified on right (8 neurons from 2 separate cultures analyzed). Scale bars, $5 \mu \mathrm{m}$.

Time in seconds on left or right of kymograph and scale bar on upper right. ${ }^{\star \star \star} p<0.0001,{ }^{\star \star \star} p<0.001$. See Figure S7 for more examples. 
A Inducible retrograde transport of clathrin-packets

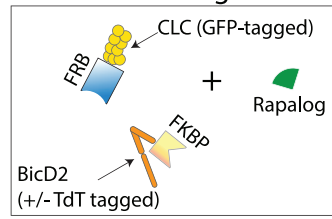

D

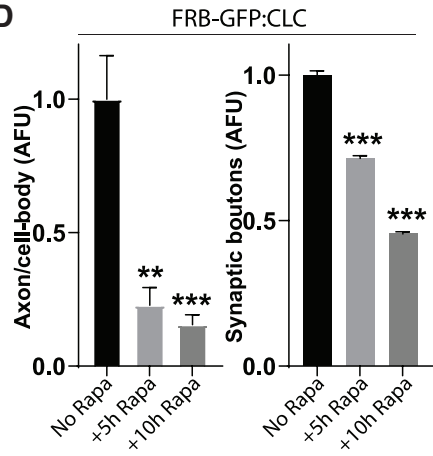

B

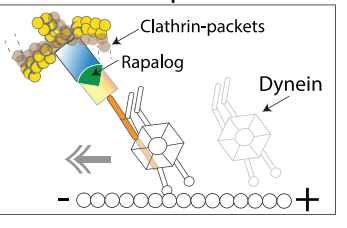

E Total clathrin

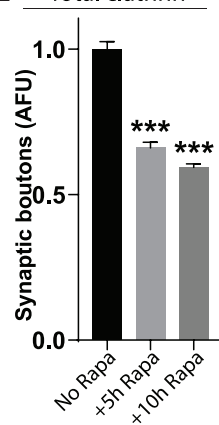

C
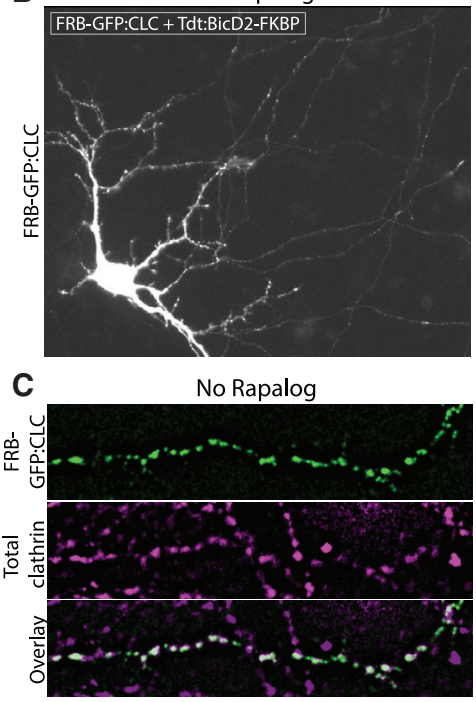
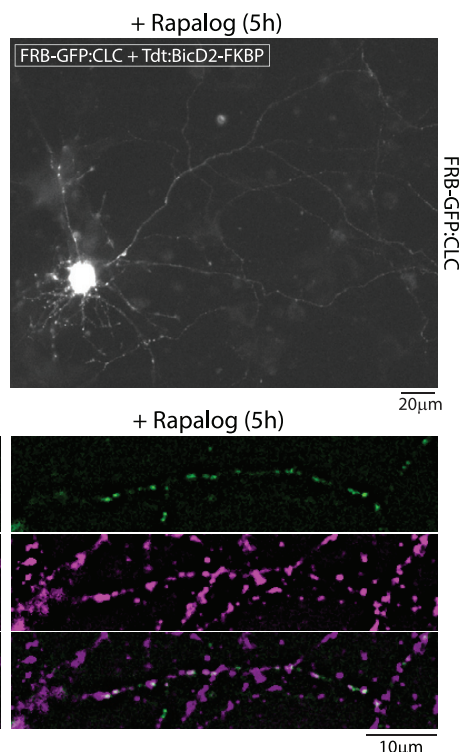

F
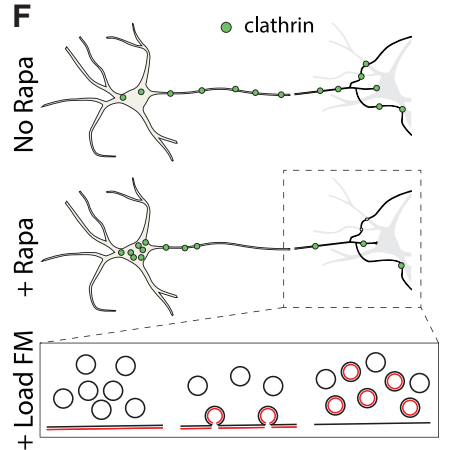

G

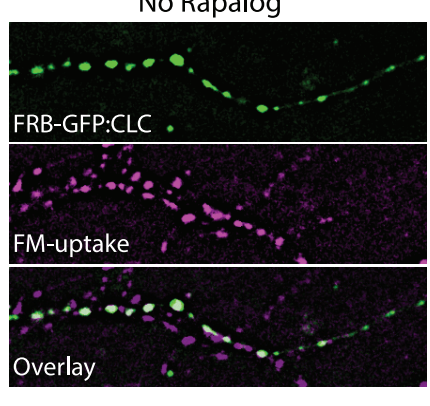

+ Rapalog (5h)

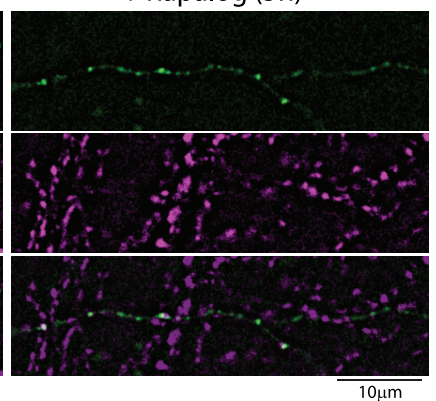

Figure 8. Depletion of synaptic clathrin packets impairs function

(A) Principle: GFP:CLC was tagged to FRB, and the dynein adaptor BicD2 was tagged to FKBP (+/- TdTomato). Upon adding Rapalog, FKBP and FRB-along with clathrin and BicD2 - would dimerize, transporting clathrin packets into the soma and depleting them from synapses.

(B) Representative images showing clustering of FRB-GFP:CLC in cell bodies after Rapalog treatment (constructs used on top left of images). Also note that transfected FRB-GFP:CLC extensively colocalizes with endogenous clathrin (Figure S8). Scale bars, $20 \mu \mathrm{m}$.

(C) Representative images showing that upon Rapalog treatment, both tagged and endogenous clathrin is depleted from boutons, quantified in (D) and (E); 9001800 boutons were analyzed for each condition from 3 separate coverslips. Scale bars, $10 \mu \mathrm{m}$.

(F) Schematic showing plan of FM-dye uptake experiments (to evaluate SV recycling) in clathrin-depleted boutons.

(G) Representative images (left) and quantification (right) showing the reduction of both clathrin and FM uptake in Rapalog-treated neurons at room temperature ( 600-950 boutons were analyzed for each condition from 3 separate cultures). Similar results were seen at $37^{\circ} \mathrm{C}$; see Figure S8E. ${ }^{* \star} \mathrm{p}<0.01,{ }^{* \star *} \mathrm{p}<0.001$. Scale bars, $10 \mu \mathrm{m}$.

delivered to distal synapses. Though previous studies in nonneuronal cells have described motile coated intermediates in the cytoplasm (Keyel et al., 2004; Puertollano et al., 2003; Rappoport and Simon, 2003; Rappoport et al., 2003a, 2003b), these seem different from the axonal transport packets. First, the intermediates are described as large, pleomorphic tubulovesicular aggregates (Polishchuk et al., 2006; Puertollano et al., 2003), different from the $\sim 40-50 \mathrm{~nm}$ uniform-sized structures we see in the axon (Figure 3). Also, the clathrin in these moving intermediates is reported to "cycle on and off"- - eventually fusing with early or late endosomes (Puertollano et al., 2003)-which is also different from the stable structures we see in axons. However, it is possible that the transported clathrin packet is a stable form of specialized coated vesicle dedicated to slow transport.
Alternatively, the transported structure might represent clathrin assemblies that are not around vesicles. Indeed, the lumens of clathrin transport packets do not appear electron dense (see Figure 3B'), though cryo-EM might be conclusive. Interestingly, "clathrin baskets" - clathrin cages with no vesicles inside-are routinely isolated from biochemical fractionations of bovine brains (Nandi et al., 1982) and, in fact, comprise $\sim 80 \%$ of the clathrin structures isolated in these preparations (Heymann et al., 2013). Clathrin baskets are thought to be artifacts, assembled in the homogenate during biochemical isolation (Heymann et al., 2013). However, given the long-lasting coated transport packets we describe here, and the large combined relative volumes that axons must occupy in brain homogenate preparations, it is conceivable 
that clathrin baskets are actually axonal transport packets. One caveat in our experiments is that our APEX-EM experiments cannot distinguish between transported and stationary clathrin in the axon.

\section{Mechanistic implications of clathrin trafficking and targeting}

The movement of clathrin between presynaptic boutons resembles SV superpools that are thought to be an extension of local SV pools (Staras et al., 2010). However, while the SV superpool is dependent on actin dynamics, the "clathrin superpool" depends on microtubules (Figure 7D) and may be a distinct superpool conveying endocytic (and perhaps other slow-component) proteins. Interestingly, the mobility of clathrin particles is also different from the soluble diffusive motion of endophilin (Bai et al., 2010), so it seems likely that there are many components to the superpool. Substantial evidence from our live-imaging, photobleaching, and photoconversion experiments supports the view that trafficking clathrin packets are captured at synapses (Figures 6 and 7) and that their depletion causes functional deficits (Figure 8); thus, it seems reasonable that the trafficking pool is critical for synaptic function. We posit that at any given synapse, multiple synaptic clathrin packets may act as local reservoirs that are "poised" to provide functional clathrin molecules that would be locally available in the vicinity of SVs, thus speeding up SV recycling. Some studies suggest that clathrin may be locally translated at axons and synapses (Biever et al., 2020; Shigeoka et al., 2016), and our experiments do not exclude a possible role for local translation, though it is clear that axonal transport is a major route for synaptic delivery of clathrin. The function of clathrin at synapses has been controversial in recent years, and clathrin has been proposed to play roles in SV biogenesis, particularly at physiologic temperatures (Delvendahl et al., 2016; Soykan et al., 2017; Watanabe et al., 2013). In that context, our key experiments were done at $\sim 37^{\circ} \mathrm{C}$, though our experiments cannot directly distinguish between the different models. Finally, the behavior of synaptic clathrin resembles the movement of soluble molecules within liquid condensates and may be conceptually related to the contemporary idea that SV clusters have fluid-like properties (Milovanovic et al., 2018). Interestingly, recent studies in non-neuronal cells indicate that CME also relies on the formation of liquid condensates (Day et al., 2021; Witkowska and Haucke, 2021), and future experiments focused on these issues might bring more clarity.

\section{$S T A R \star M E T H O D S$}

Detailed methods are provided in the online version of this paper and include the following:

- KEY RESOURCES TABLE

- RESOURCE AVAILABILITY

○ Lead contact

O Materials availability statement

D Data and code availability

- EXPERIMENTAL MODEL AND SUBJECT DETAILS

O Animals

O Primary cell cultures
- METHOD DETAILS

O DNA constructs, pharmacologic agents, neuronal cultures, transfections, and immunocytochemistry

$\bigcirc$ Live imaging, photobleaching, photoactivation, and photoconversion experiments

O Rapamycin-inducible FKBP-FRB dimerizer and FM464 assays

O Biochemical fractionation, western blotting and immuno-precipitation from mouse sciatic nerves

- Protein identification through MudPIT-MS analysis and in silico data analysis

O Sample preparation, data acquisition and data processing for electron tomography

$\bigcirc$ DNA-PAINT imaging and image analysis

- QUANTIFICATION AND STATISTICAL ANALYSIS

○ FRAP Quantification

O Statistical Analyses

\section{SUPPLEMENTAL INFORMATION}

Supplemental information can be found online at https://doi.org/10.1016/j. neuron.2021.08.016.

\section{ACKNOWLEDGMENTS}

This work was supported by a NIH grant to S.R. (R01NS075233), a CNRS ATIP grant to C.L. (ATIP2016), NIH grants to D.B. (R01GM086197) and M.H.E. (P41 GM103412) supporting the National Center for Microscopy and Imaging Research, and NIH grants to J.R.Y. (P41 GM103533 and R01 MH100175). The authors thank Titus Jung for proteomic data handling and various investigators for generously sharing constructs (listed in STAR methods).

\section{AUTHOR CONTRIBUTIONS}

S.R. conceived the project. A.G., R.S., and S.R. designed most of the experiments and analyzed the data. C.L., F.W., and G.C. designed and performed the DNA-PAINT experiments and data analyses. A.G. performed the live-imaging experiments in axons and dendrites and analyzed most of the ensuing data. Electron microscopy/analysis was performed by A.G., with assistance from D.B. and S.P. at NCMIR, led by M.H.E. Samples for mass spectrometry were prepared by A.G.; experiments were performed by X.H. under the supervision of J.R.Y.; and ensuing data were analyzed by X.H. and A.G. R.S. performed the synaptic live imaging, photo-manipulation, FKBP/FRB/FM uptake, and live/dead assay experiments; analyzed the ensuing data; and helped analyze some of the axonal transport data. N.P.B. designed strategies to analyze the photo-manipulation experiments and performed most of the ensuing analyses, with assistance from R.S. and S.R. L.P. helped with neuronal cultures, and U.D. and L.P. designed and generated constructs. S.R. wrote the manuscript, with contributions from C.L., R.S., and A.G.

\section{DECLARATION OF INTERESTS}

The authors declare no competing interests.

Received: March 1, 2020

Revised: June 10, 2021

Accepted: August 13, 2021

Published: September 15, 2021

\section{REFERENCES}

Applewhite, D.A., Barzik, M., Kojima, S., Svitkina, T.M., Gertler, F.B., and Borisy, G.G. (2007). Ena/VASP proteins have an anti-capping independent function in filopodia formation. Mol. Biol. Cell 18, 2579-2591. 
Bai, J., Hu, Z., Dittman, J.S., Pym, E.C., and Kaplan, J.M. (2010). Endophilin functions as a membrane-bending molecule and is delivered to endocytic zones by exocytosis. Cell 143, 430-441.

Bazinet, C., Katzen, A.L., Morgan, M., Mahowald, A.P., and Lemmon, S.K. (1993). The Drosophila clathrin heavy chain gene: clathrin function is essential in a multicellular organism. Genetics 134, 1119-1134.

Belshaw, P.J., Ho, S.N., Crabtree, G.R., and Schreiber, S.L. (1996). Controlling protein association and subcellular localization with a synthetic ligand that induces heterodimerization of proteins. Proc. Natl. Acad. Sci. USA 93, 4604-4607.

Bennett, E.M., Chen, C.Y., Engqvist-Goldstein, A.E., Drubin, D.G., and Brodsky, F.M. (2001). Clathrin hub expression dissociates the actin-binding protein Hip1R from coated pits and disrupts their alignment with the actin cytoskeleton. Traffic 2, 851-858.

Bentley, M., Decker, H., Luisi, J., and Banker, G. (2015). A novel assay reveals preferential binding between Rabs, kinesins, and specific endosomal subpopulations. J. Cell Biol. 208, 273-281.

Biever, A., Glock, C., Tushev, G., Ciirdaeva, E., Dalmay, T., Langer, J.D., and Schuman, E.M. (2020). Monosomes actively translate synaptic mRNAs in neuronal processes. Science 367, eaay4991. https://doi.org/10.1126/science.aay4991.

Black, M.M., Chestnut, M.H., Pleasure, I.T., and Keen, J.H. (1991). Stable clathrin: uncoating protein (hsc70) complexes in intact neurons and their axonal transport. J. Neurosci. 11, 1163-1172.

Blanpied, T.A., Scott, D.B., and Ehlers, M.D. (2002). Dynamics and regulation of clathrin coats at specialized endocytic zones of dendrites and spines. Neuron 36, 435-449.

Boyer, N.P., McCormick, L.E., Menon, S., Urbina, F.L., and Gupton, S.L. (2020). A pair of E3 ubiquitin ligases compete to regulate filopodial dynamics and axon guidance. J. Cell Biol. 219, e201902088.

Burkel, B.M., von Dassow, G., and Bement, W.M. (2007). Versatile fluorescent probes for actin filaments based on the actin-binding domain of utrophin. Cell Motil. Cytoskeleton 64, 822-832.

Bushlin, I., Petralia, R.S., Wu, F., Harel, A., Mughal, M.R., Mattson, M.P., and Yao, P.J. (2008). Clathrin assembly protein AP180 and CALM differentially control axogenesis and dendrite outgrowth in embryonic hippocampal neurons. J. Neurosci. 28, 10257-10271.

Chakrabarty, N., Dubey, P., Tang, Y., Ganguly, A., Ladt, K., Leterrier, C., Jung, P., and Roy, S. (2019). Processive flow by biased polymerization mediates the slow axonal transport of actin. J. Cell Biol. 218, 112-124.

Chanaday, N.L., and Kavalali, E.T. (2018). Optical detection of three modes of endocytosis at hippocampal synapses. eLife 7, e36097.

Chanaday, N.L., Cousin, M.A., Milosevic, I., Watanabe, S., and Morgan, J.R. (2019). The Synaptic Vesicle Cycle Revisited: New Insights into the Modes and Mechanisms. J. Neurosci. 39, 8209-8216.

Cousin, M.A., Gordon, S.L., and Smillie, K.J. (2018). Using FM dyes to monitor clathrin-mediated endocytosis in primary neuronal culture. Clathrin-Mediated Endocytosis (Humana Press), pp. 239-249.

Darcy, K.J., Staras, K., Collinson, L.M., and Goda, Y. (2006). Constitutive sharing of recycling synaptic vesicles between presynaptic boutons. Nat. Neurosci. 9, 315-321.

Das, U., Scott, D.A., Ganguly, A., Koo, E.H., Tang, Y., and Roy, S. (2013). Activity-induced convergence of APP and BACE-1 in acidic microdomains via an endocytosis-dependent pathway. Neuron 79, 447-460.

Day, K.J., Kago, G., Wang, L., Richter, J.B., Hayden, C.C., Lafer, E.M., and Stachowiak, J.C. (2021). Liquid-like protein interactions catalyse assembly of endocytic vesicles. Nat. Cell Biol. 23, 366-376.

de Waegh, S., and Brady, S.T. (1989). Axonal transport of a clathrin uncoating ATPase (HSC70): a role for $\mathrm{HSC} 70$ in the modulation of coated vesicle assembly in vivo. J. Neurosci. Res. 23, 433-440.

Delvendahl, I., Vyleta, N.P., von Gersdorff, H., and Hallermann, S. (2016). Fast, Temperature-Sensitive and Clathrin-Independent Endocytosis at Central Synapses. Neuron 90, 492-498.
Denker, A., and Rizzoli, S.O. (2010). Synaptic vesicle pools: an update. Front. Synaptic Neurosci. 2, 135.

Dienstbier, M., and Li, X. (2009). Bicaudal-D and its role in cargo sorting by microtubule-based motors. Biochem. Soc. Trans. 37, 1066-1071.

Dittman, J., and Ryan, T.A. (2009). Molecular circuitry of endocytosis at nerve terminals. Annu. Rev. Cell Dev. Biol. 25, 133-160.

Elluru, R.G., Bloom, G.S., and Brady, S.T. (1995). Fast axonal transport of kinesin in the rat visual system: functionality of kinesin heavy chain isoforms. Mol. Biol. Cell 6, 21-40.

Gaffield, M.A., and Betz, W.J. (2006). Imaging synaptic vesicle exocytosis and endocytosis with FM dyes. Nat. Protoc. 1, 2916-2921.

Gaidarov, I., Santini, F., Warren, R.A., and Keen, J.H. (1999). Spatial control of coated-pit dynamics in living cells. Nat. Cell Biol. 1, 1-7.

Ganguly, A., and Roy, S. (2014). Using photoactivatable GFP to track axonal transport kinetics. Methods Mol. Biol. 1148, 203-215.

Ganguly, A., Tang, Y., Wang, L., Ladt, K., Loi, J., Dargent, B., Leterrier, C., and Roy, S. (2015). A dynamic formin-dependent deep F-actin network in axons. J. Cell Biol. 210, 401-417.

Ganguly, A., Han, X., Das, U., Wang, L., Loi, J., Sun, J., Gitler, D., Caillol, G., Leterrier, C., Yates, J.R., 3rd, and Roy, S. (2017). Hsc70 chaperone activity is required for the cytosolic slow axonal transport of synapsin. J. Cell Biol. 216, 2059-2074.

Garner, J.A., and Lasek, R.J. (1981). Clathrin is axonally transported as part of slow component b: the microfilament complex. J. Cell Biol. 88, 172-178.

Gerrow, K., Romorini, S., Nabi, S.M., Colicos, M.A., Sala, C., and El-Husseini, A. (2006). A preformed complex of postsynaptic proteins is involved in excitatory synapse development. Neuron 49, 547-562.

Gitler, D., Xu, Y., Kao, H.T., Lin, D., Lim, S., Feng, J., Greengard, P., and Augustine, G.J. (2004). Molecular determinants of synapsin targeting to presynaptic terminals. J. Neurosci. 24, 3711-3720.

Goddard, T.D., Huang, C.C., Meng, E.C., Pettersen, E.F., Couch, G.S., Morris, J.H., and Ferrin, T.E. (2018). UCSF ChimeraX: Meeting modern challenges in visualization and analysis. Protein Sci. 27, 14-25.

Gower, D.J., and Tytell, M. (1987). Axonal transport of clathrin-associated proteins. Brain Res. 407, 1-8.

Heuser, J.E., and Reese, T.S. (1973). Evidence for recycling of synaptic vesicle membrane during transmitter release at the frog neuromuscular junction. J. Cell Biol. 57, 315-344.

Heymann, J.B., Winkler, D.C., Yim, Y.I., Eisenberg, E., Greene, L.E., and Steven, A.C. (2013). Clathrin-coated vesicles from brain have small payloads: a cryo-electron tomographic study. J. Struct. Biol. 184, 43-51.

Jimenez, A., Friedl, K., and Leterrier, C. (2020). About samples, giving examples: Optimized Single Molecule Localization Microscopy. Methods 174, 100-114.

Jung, N., and Haucke, V. (2007). Clathrin-mediated endocytosis at synapses. Traffic 8, 1129-1136.

Jungmann, R., Avendaño, M.S., Woehrstein, J.B., Dai, M., Shih, W.M., and Yin, P. (2014). Multiplexed 3D cellular super-resolution imaging with DNA-PAINT and Exchange-PAINT. Nat. Methods 11, 313-318.

Kaksonen, M., Toret, C.P., and Drubin, D.G. (2006). Harnessing actin dynamics for clathrin-mediated endocytosis. Nat. Rev. Mol. Cell Biol. 7, 404-414. Kapitein, L.C., Schlager, M.A., van der Zwan, W.A., Wulf, P.S., Keijzer, N., and Hoogenraad, C.C. (2010). Probing intracellular motor protein activity using an inducible cargo trafficking assay. Biophys. J. 99, 2143-2152.

Keyel, P.A., Watkins, S.C., and Traub, L.M. (2004). Endocytic adaptor molecules reveal an endosomal population of clathrin by total internal reflection fluorescence microscopy. J. Biol. Chem. 279, 13190-13204.

Kononenko, N.L., Puchkov, D., Classen, G.A., Walter, A.M., Pechstein, A., Sawade, L., Kaempf, N., Trimbuch, T., Lorenz, D., Rosenmund, C., et al. (2014). Clathrin/AP-2 mediate synaptic vesicle reformation from endosomelike vacuoles but are not essential for membrane retrieval at central synapses. Neuron 82, 981-988. 
Kremer, J.R., Mastronarde, D.N., and McIntosh, J.R. (1996). Computer visualization of three-dimensional image data using IMOD. J. Struct. Biol. 116, 71-76.

Krueger, S.R., Kolar, A., and Fitzsimonds, R.M. (2003). The presynaptic release apparatus is functional in the absence of dendritic contact and highly mobile within isolated axons. Neuron 40, 945-957.

Lawrence, A., Bouwer, J.C., Perkins, G., and Ellisman, M.H. (2006). Transformbased backprojection for volume reconstruction of large format electron microscope tilt series. J. Struct. Biol. 154, 144-167.

Leterrier, C., Dubey, P., and Roy, S. (2017). The nano-architecture of the axonal cytoskeleton. Nat. Rev. Neurosci. 18, 713-726.

Liao, L., Park, S.K., Xu, T., Vanderklish, P., and Yates, J.R., 3rd (2008). Quantitative proteomic analysis of primary neurons reveals diverse changes in synaptic protein content in fmr1 knockout mice. Proc. Natl. Acad. Sci. USA 105, 15281-15286.

Liljebäck, H., Quach, M., Carlsson, P.-O., and Lau, J. (2019). Fewer Islets Survive from a First Transplant than a Second Transplant: Evaluation of Repeated Intraportal Islet Transplantation in Mice. Cell Transplant. 28, 1455-1460.

Lippincott-Schwartz, J., Altan-Bonnet, N., and Patterson, G.H. (2003). Photobleaching and photoactivation: following protein dynamics in living cells. Nat. Cell Biol. Suppl (Suppl), S7-S14.

Liu, S.H., Marks, M.S., and Brodsky, F.M. (1998). A dominant-negative clathrin mutant differentially affects trafficking of molecules with distinct sorting motifs in the class II major histocompatibility complex (MHC) pathway. J. Cell Biol. 140, 1023-1037.

Macia, E., Ehrlich, M., Massol, R., Boucrot, E., Brunner, C., and Kirchhausen, T. (2006). Dynasore, a cell-permeable inhibitor of dynamin. Dev. Cell 10, 839-850.

Maday, S., Twelvetrees, A.E., Moughamian, A.J., and Holzbaur, E.L. (2014). Axonal transport: cargo-specific mechanisms of motility and regulation. Neuron 84, 292-309.

Martell, J.D., Deerinck, T.J., Sancak, Y., Poulos, T.L., Mootha, V.K., Sosinsky, G.E., Ellisman, M.H., and Ting, A.Y. (2012). Engineered ascorbate peroxidase as a genetically encoded reporter for electron microscopy. Nat. Biotechnol. 30, 1143-1148.

Massol, R.H., Boll, W., Griffin, A.M., and Kirchhausen, T. (2006). A burst of auxilin recruitment determines the onset of clathrin-coated vesicle uncoating. Proc. Natl. Acad. Sci. USA 103, 10265-10270.

Mastronarde, D.N. (2005). Automated electron microscope tomography using robust prediction of specimen movements. J. Struct. Biol. 152, 36-51.

McDonald, W.H., Tabb, D.L., Sadygov, R.G., MacCoss, M.J., Venable, J., Graumann, J., Johnson, J.R., Cociorva, D., and Yates, J.R., 3rd (2004). MS1, MS2, and SQT-three unified, compact, and easily parsed file formats for the storage of shotgun proteomic spectra and identifications. Rapid Commun. Mass Spectrom. 18, 2162-2168, 2168.g.

McMahon, H.T., and Boucrot, E. (2011). Molecular mechanism and physiological functions of clathrin-mediated endocytosis. Nat. Rev. Mol. Cell Biol. 12, 517-533.

Milosevic, I. (2018). Revisiting the Role of Clathrin-Mediated Endoytosis in Synaptic Vesicle Recycling. Front. Cell. Neurosci. 12, 27.

Milovanovic, D., Wu, Y., Bian, X., and De Camilli, P. (2018). A liquid phase of synapsin and lipid vesicles. Science 361, 604-607.

Mueller, V.J., Wienisch, M., Nehring, R.B., and Klingauf, J. (2004). Monitoring clathrin-mediated endocytosis during synaptic activity. J. Neurosci. 24, 2004-2012.

Nandi, P.K., Irace, G., Van Jaarsveld, P.P., Lippoldt, R.E., and Edelhoch, H. (1982). Instability of coated vesicles in concentrated sucrose solutions. Proc. Natl. Acad. Sci. USA 79, 5881-5885.

Paraan, M., Mendez, J., Sharum, S., Kurtin, D., He, H., and Stagg, S.M. (2020). The structures of natively assembled clathrin-coated vesicles. Sci. Adv. 6, a8397.
Patterson, G.H., and Lippincott-Schwartz, J. (2002). A photoactivatable GFP for selective photolabeling of proteins and cells. Science 297, 1873-1877.

Pelassa, I., Zhao, C., Pasche, M., Odermatt, B., and Lagnado, L. (2014). Synaptic vesicles are "primed" for fast clathrin-mediated endocytosis at the ribbon synapse. Front. Mol. Neurosci. 7, 91.

Peng, J., Kim, M.J., Cheng, D., Duong, D.M., Gygi, S.P., and Sheng, M. (2004). Semiquantitative proteomic analysis of rat forebrain postsynaptic density fractions by mass spectrometry. J. Biol. Chem. 279, 21003-21011.

Perez-Riverol, Y., Xu, Q.W., Wang, R., Uszkoreit, J., Griss, J., Sanchez, A., Reisinger, F., Csordas, A., Ternent, T., Del-Toro, N., et al. (2016). PRIDE Inspector Toolsuite: Moving Toward a Universal Visualization Tool for Proteomics Data Standard Formats and Quality Assessment of ProteomeXchange Datasets. Mol. Cell. Proteomics 15, 305-317.

Petralia, R.S., Wang, Y.X., and Wenthold, R.J. (2003). Internalization at glutamatergic synapses during development. Eur. J. Neurosci. 18, 3207-3217.

Phan, S., Boassa, D., Nguyen, P., Wan, X., Lanman, J., Lawrence, A., and Ellisman, M.H. (2017). 3D reconstruction of biological structures: automated procedures for alignment and reconstruction of multiple tilt series in electron tomography. Adv. Struct. Chem. Imaging 2, 8.

Polishchuk, R.S., San Pietro, E., Di Pentima, A., Teté, S., and Bonifacino, J.S. (2006). Ultrastructure of long-range transport carriers moving from the trans Golgi network to peripheral endosomes. Traffic 7, 1092-1103.

Puertollano, R., van der Wel, N.N., Greene, L.E., Eisenberg, E., Peters, P.J., and Bonifacino, J.S. (2003). Morphology and dynamics of clathrin/GGA1coated carriers budding from the trans-Golgi network. Mol. Biol. Cell 14, 1545-1557.

Radulescu, A.E., Siddhanta, A., and Shields, D. (2007). A role for clathrin in reassembly of the Golgi apparatus. Mol. Biol. Cell 18, 94-105.

Rappoport, J.Z., and Simon, S.M. (2003). Real-time analysis of clathrin-mediated endocytosis during cell migration. J. Cell Sci. 116, 847-855.

Rappoport, J.Z., Taha, B.W., Lemeer, S., Benmerah, A., and Simon, S.M. (2003a). The AP-2 complex is excluded from the dynamic population of plasma membrane-associated clathrin. J. Biol. Chem. 278, 47357-47360.

Rappoport, J.Z., Taha, B.W., and Simon, S.M. (2003b). Movement of plasmamembrane-associated clathrin spots along the microtubule cytoskeleton. Traffic 4, 460-467.

Ratnayaka, A., Marra, V., Branco, T., and Staras, K. (2011). Extrasynaptic vesicle recycling in mature hippocampal neurons. Nat. Commun. 2, 531.

Robinson, M.S., Sahlender, D.A., and Foster, S.D. (2010). Rapid inactivation of proteins by rapamycin-induced rerouting to mitochondria. Dev. Cell 18, 324-331.

Rosendale, M., Jullié, D., Choquet, D., and Perrais, D. (2017). Spatial and Temporal Regulation of Receptor Endocytosis in Neuronal Dendrites Revealed by Imaging of Single Vesicle Formation. Cell Rep. 18, 1840-1847.

Roy, S. (2014). Seeing the unseen: the hidden world of slow axonal transport. Neuroscientist 20, 71-81.

Roy, S. (2020). Finding order in slow axonal transport. Curr. Opin. Neurobiol. 63, 87-94.

Roy, S., Yang, G., Tang, Y., and Scott, D.A. (2011). A simple photoactivation and image analysis module for visualizing and analyzing axonal transport with high temporal resolution. Nat. Protoc. 7, 62-68.

Royle, S.J., and Lagnado, L. (2010). Clathrin-mediated endocytosis at the synaptic terminal: bridging the gap between physiology and molecules. Traffic 11, 1489-1497.

Saheki, Y., and De Camilli, P. (2012). Synaptic vesicle endocytosis. Cold Spring Harb. Perspect. Biol. 4, a005645.

Schindelin, J., Arganda-Carreras, I., Frise, E., Kaynig, V., Longair, M., Pietzsch, T., Preibisch, S., Rueden, C., Saalfeld, S., Schmid, B., et al. (2012). Fiji: an open-source platform for biological-image analysis. Nat. Methods 9, 676-682.

Scott, D.A., Das, U., Tang, Y., and Roy, S. (2011). Mechanistic logic underlying the axonal transport of cytosolic proteins. Neuron 70, 441-454. 
Sharma, R., Gulia, R., and Bhattacharyya, S. (2019). Analysis of ubiquitination and ligand-dependent trafficking of group I mGluRs. Methods Cell Biol. 149, 107-130.

Shigeoka, T., Jung, H., Jung, J., Turner-Bridger, B., Ohk, J., Lin, J.Q., Amieux, P.S., and Holt, C.E. (2016). Dynamic Axonal Translation in Developing and Mature Visual Circuits. Cell 166, 181-192.

Song, B.D., Yarar, D., and Schmid, S.L. (2004). An assembly-incompetent mutant establishes a requirement for dynamin self-assembly in clathrin-mediated endocytosis in vivo. Mol. Biol. Cell 15, 2243-2252.

Soykan, T., Kaempf, N., Sakaba, T., Vollweiter, D., Goerdeler, F., Puchkov, D. Kononenko, N.L., and Haucke, V. (2017). Synaptic Vesicle Endocytosis Occurs on Multiple Timescales and Is Mediated by Formin-Dependent Actin Assembly. Neuron 93, 854-866.e854.

Staras, K., Branco, T., Burden, J.J., Pozo, K., Darcy, K., Marra, V., Ratnayaka, A., and Goda, Y. (2010). A vesicle superpool spans multiple presynaptic terminals in hippocampal neurons. Neuron 66, 37-44.

Tabb, D.L., McDonald, W.H., and Yates, J.R., 3rd (2002). DTASelect and Contrast: tools for assembling and comparing protein identifications from shotgun proteomics. J. Proteome Res. 1, 21-26.

Tang, Y., Das, U., Scott, D.A., and Roy, S. (2012). The slow axonal transport of alpha-synuclein-mechanistic commonalities amongst diverse cytosolic cargoes. Cytoskeleton (Hoboken) 69, 506-513.

Tang, Y., Scott, D., Das, U., Gitler, D., Ganguly, A., and Roy, S. (2013). Fast vesicle transport is required for the slow axonal transport of synapsin. J. Neurosci. 33, 15362-15375.

Tao-Cheng, J.H. (2020). Stimulation-induced differential redistributions of clathrin and clathrin-coated vesicles in axons compared to soma/dendrites. Mol. Brain 13, 141.

Traub, L.M., and Bonifacino, J.S. (2013). Cargo recognition in clathrin-mediated endocytosis. Cold Spring Harb. Perspect. Biol. 5, a016790.

Twelvetrees, A.E., Pernigo, S., Sanger, A., Guedes-Dias, P., Schiavo, G. Steiner, R.A., Dodding, M.P., and Holzbaur, E.L. (2016). The Dynamic
Localization of Cytoplasmic Dynein in Neurons Is Driven by Kinesin-1. Neuron 90, 1000-1015.

Wang, L., Das, U., Scott, D.A., Tang, Y., McLean, P.J., and Roy, S. (2014). $\alpha$-synuclein multimers cluster synaptic vesicles and attenuate recycling. Curr. Biol. 24, 2319-2326.

Washburn, M.P., Wolters, D., and Yates, J.R., 3rd (2001). Large-scale analysis of the yeast proteome by multidimensional protein identification technology. Nat. Biotechnol. 19, 242-247.

Watanabe, S., Rost, B.R., Camacho-Pérez, M., Davis, M.W., Söhl-Kielczynski, B., Rosenmund, C., and Jorgensen, E.M. (2013). Ultrafast endocytosis at mouse hippocampal synapses. Nature 504, 242-247.

Watanabe, S., Trimbuch, T., Camacho-Pérez, M., Rost, B.R., Brokowski, B., Söhl-Kielczynski, B., Felies, A., Davis, M.W., Rosenmund, C., and Jorgensen, E.M. (2014). Clathrin regenerates synaptic vesicles from endosomes. Nature 515, 228-233.

Wilhelm, B.G., Mandad, S., Truckenbrodt, S., Kröhnert, K., Schäfer, C., Rammner, B., Koo, S.J., Claßen, G.A., Krauss, M., Haucke, V., et al. (2014) Composition of isolated synaptic boutons reveals the amounts of vesicle trafficking proteins. Science 344, 1023-1028.

Witkowska, A., and Haucke, V. (2021). Liquid-like protein assemblies initiate endocytosis. Nat. Cell Biol. 23, 301-302.

Xu, T., Park, S.K., Venable, J.D., Wohlschlegel, J.A., Diedrich, J.K., Cociorva D., Lu, B., Liao, L., Hewel, J., Han, X., et al. (2015). ProLuCID: An improved SEQUEST-like algorithm with enhanced sensitivity and specificity. J. Proteomics 129, 16-24.

Yates, J.R., Ruse, C.I., and Nakorchevsky, A. (2009). Proteomics by mass spectrometry: approaches, advances, and applications. Annu. Rev. Biomed. Eng. 11, 49-79.

Zhao, Y., and Keen, J.H. (2008). Gyrating clathrin: highly dynamic clathrin structures involved in rapid receptor recycling. Traffic 9, 2253-2264. 


\section{STAR $\star$ METHODS}

\section{KEY RESOURCES TABLE}

\begin{tabular}{|c|c|c|}
\hline REAGENT or RESOURCE & SOURCE & IDENTIFIER \\
\hline \multicolumn{3}{|l|}{ Antibodies } \\
\hline Anti-Clathrin heavy chain antibody & Abcam & Cat\#ab21679; RRID:AB_2083165 \\
\hline Chicken polyclonal anti-GFP antibody & Abcam & Cat\#ab13970; RRID:AB_300798 \\
\hline Anti-rabbit Alexa Fluor 594 & Invitrogen & Cat\#A11037; RRID:AB_2534095 \\
\hline Anti-Chicken Alexa Fluor 488 & Invitrogen & Cat\#A11039; RRID:AB_142924 \\
\hline polyclonal chicken anti-Map2 & Abcam & Cat\# ab5392; RRID:AB_2138153 \\
\hline monoclonal mouse anti $\beta 2$-spectrin & BD Bioscience & Cat\#612563; RRID:AB_399854 \\
\hline Anti-Clathrin mouse monoclonal antibody & Affinity Bioreagents & Cat\#MA1-065; RRID:AB_2083179 \\
\hline Anti-Mouse IgG & Abcam & Cat\#ab190475; RRID:AB_2827162 \\
\hline monoclonal mouse anti-synapsin & Synaptic Systems & Cat\#106-001; RRID:AB_887805 \\
\hline Anti-rabbit D2 (Ultivue Kit) & Ultivue & Cat\#Ultivue-2 \\
\hline Anti-mouse D2 (Ultivue Kit) & Ultivue & Cat\#Ultivue-2 \\
\hline \multicolumn{3}{|l|}{ Chemicals, peptides, and recombinant proteins } \\
\hline Dynasore & Abcam & Cat\#ab120192 \\
\hline Latrunculin A & Life Technologies & Cat\#L12370 \\
\hline Nocodazole & Millipore-Sigma & Cat\#487928-10MG \\
\hline Swinholide A & Millipore-Sigma & Cat\#574776-10UG \\
\hline $\mathrm{A} / \mathrm{C}$ heterodimerizer & Takara & Cat\#635056 \\
\hline FM4-64 & Invitrogen & Cat\#T13320 \\
\hline Advasep-7 & Millipore-Sigma & Cat\#A3723 \\
\hline HBSS & GIBCO & Cat\#14025092 \\
\hline $\begin{array}{l}\text { 6-cyano-7-nitroquinoxaline-2,3- } \\
\text { dione CNQX }\end{array}$ & Tocris Biosciences & Cat\#1045 \\
\hline D,L-2-amino-5-phosphonovaleric acid AP5 & Tocris Biosciences & Cat\#106 \\
\hline VER155008 & Tocris Biosciences & Cat\#3803 \\
\hline Lipofectamine 2000 & Life Technologies & Cat\#11668-019 \\
\hline $\begin{array}{l}\text { ProFection Mammalian Transfection } \\
\text { System }\end{array}$ & Promega & Cat\#E1200 \\
\hline B27 supplement & GIBCO & Cat\#17504044 \\
\hline Glutamax & GIBCO & Cat\#35050061 \\
\hline Neurobasal A & GIBCO & Cat\#10888022 \\
\hline poly-D-lysine & Sigma & Cat\#P0899 \\
\hline Hibernate-E low fluorescence medium & Brainbits & Cat\#HE-If \\
\hline $0.25 \%$ Trypsin-EDTA & Invitrogen & Cat\#25200072 \\
\hline Fetal bovine serum & Hyclone & Cat\#SH30071.03 \\
\hline Hemin chloride & Millipore-Sigma & Cat\#3741 \\
\hline Sodium cacodylate trihydrate & Millipore-Sigma & Cat\#C4945 \\
\hline Diaminobenzidine (DAB) & Millipore-Sigma & Cat\#D8001 \\
\hline Osmium Tetraoxide ( $4 \%$ aqueous solution) & Electron Microscopy Sciences & Cat\#19150 \\
\hline Paraformaldehyde ( $16 \%$ aqueous solution) & Electron Microscopy Sciences & Cat\#15710 \\
\hline Glutaraldehyde (25\% aqueous solution) & Electron Microscopy Sciences & Cat\#16220 \\
\hline Durcupan ACM, Epoxy Resin (Kit) & Electron Microscopy Sciences & Cat\#14040 \\
\hline $\begin{array}{l}\text { Tris (2-carboxyethyl) phosphine } \\
\text { hydrochloride }\end{array}$ & Sigma-Aldrich & Cat\# 75259 \\
\hline
\end{tabular}




\begin{tabular}{|c|c|c|}
\hline \multicolumn{3}{|l|}{ Continued } \\
\hline REAGENT or RESOURCE & SOURCE & IDENTIFIER \\
\hline lodo-acetamide & Sigma-Aldrich & Cat\#144-48-9 \\
\hline Protease inhibitor cocktail & Sigma-Aldrich & Cat\#P8340 \\
\hline Dynabeads Co-Immunoprecipitation Kit & ThermoFisher Scientific & Cat\#14321D \\
\hline $1.4 \mathrm{~nm}$ Nanogold secondary antibody & Nanoprobes & Cat\#2001 \\
\hline $\mathrm{HQ}$ silver enhancement kit & Nanoprobes & Cat\#2012-45ML \\
\hline \multicolumn{3}{|l|}{ Critical commercial assays } \\
\hline Live-or-Dye NucFix Red & Biotium & Cat\#32010-T \\
\hline \multicolumn{3}{|l|}{ Deposited data } \\
\hline Proteomics Data & $\begin{array}{l}\text { This Paper; ProteomeXchange } \\
\text { Consortium (PRIDE database) } \\
\text { (Perez-Riverol et al., 2016) }\end{array}$ & $\begin{array}{l}\text { Table S1; PXD027857 (https://www.ebi.ac. } \\
\text { uk/pride/) }\end{array}$ \\
\hline \multicolumn{3}{|l|}{ Experimental models: Organisms/strains } \\
\hline Mouse: CD1 & Charles River Laboratories & Cat\#022-CD1 \\
\hline \multicolumn{3}{|l|}{ Recombinant DNA } \\
\hline GFP:CLC & Gaidarov et al.,1999 & N/A \\
\hline pUC57-APEX & Martell et al., 2012 & Addgene Plasmid \#40306 \\
\hline Dendra2-Lifeact-7 & $\begin{array}{l}\text { Dendra2-Lifeact-7 was } \\
\text { a gift from Michael Davidson }\end{array}$ & Addgene plasmid \#54694 \\
\hline Dendra2:CLC & This paper & $\mathrm{N} / \mathrm{A}$ \\
\hline PAGFP-C1 & $\begin{array}{l}\text { Patterson and Lippincott- } \\
\text { Schwartz, } 2002\end{array}$ & Addgene plasmid \#11910 \\
\hline PAGFP:CLC & This paper & $\mathrm{N} / \mathrm{A}$ \\
\hline CHC-T7-Hub & Liu et al., 1998 & $\mathrm{~N} / \mathrm{A}$ \\
\hline T7-empty backbone & Liu et al., 1998 & N/A \\
\hline GFP:Utr-CH & Burkel et al., 2007 & Addgene Plasmid \#26737 \\
\hline synaptophysin:mCh & Gerrow et al., 2006 & N/A \\
\hline pBa-Flag-BicD2-FKBP & Bentley et al., 2015 & Addgene Plasmid \#64206 \\
\hline pBa- Flag-tdTM-BicD2-FKBP & Bentley et al., 2015 & Addgene Plasmid \#64205 \\
\hline pBa-FRB-3myc-Rab5a & Bentley et al., 2015 & Addgene Plasmid \#64209 \\
\hline FRB-GFP:CLC & This paper & N/A \\
\hline GFP:synapsin-1a & Gitler et al., 2004 & $\mathrm{~N} / \mathrm{A}$ \\
\hline EGFP-AP180 & Bushlin et al., 2008 & $\mathrm{~N} / \mathrm{A}$ \\
\hline GAK-GFP & Massol et al., 2006 & N/A \\
\hline pEGFP-C1-Dynamin & Song et al., 2004 & Addgene Plasmid \#34680 \\
\hline CLC:mCherry & This paper & N/A \\
\hline pEGFP-N1 & Clonetech & Cat\#6085-1 \\
\hline \multicolumn{3}{|l|}{ Software and algorithms } \\
\hline GraphPad Prism & $\begin{array}{l}\text { GraphPad Software, } \\
\text { La Jolla, CA, USA }\end{array}$ & $\begin{array}{l}\text { Version 7.00; www.graphpad.com; } \\
\text { RRID:SCR_002798 }\end{array}$ \\
\hline Fiji ImageJ & Schindelin et al., 2012 & https://imagej.net/Fiji; RRID:SCR_002285 \\
\hline MetaMorph Version 7.10 & Molecular Devices & $\begin{array}{l}\text { https://www.moleculardevices.com/ } \\
\text { products/cellular-imaging-systems/ } \\
\text { acquisition-and-analysis-software/ } \\
\text { metamorph-microscopy; } \\
\text { RRID:SCR_002368 }\end{array}$ \\
\hline Adobe Illustrator & Adobe & $\begin{array}{l}\text { https://www.adobe.com/products/ } \\
\text { illustrator/free-trial-download.html; } \\
\text { RRID:SCR_010279 }\end{array}$ \\
\hline RawXtract version 1.9 .9 & McDonald et al., 2004 & http://fields.scripps.edu/downloads.php \\
\hline
\end{tabular}




\begin{tabular}{|c|c|c|}
\hline \multicolumn{3}{|l|}{ Continued } \\
\hline REAGENT or RESOURCE & SOURCE & IDENTIFIER \\
\hline N-STORM software & Nikon; Jungmann et al., 2014 & $\begin{array}{l}\text { https:// } \\
\text { www.microscope.healthcare.nikon.com/ } \\
\text { products/super-resolution-microscopes/n- } \\
\text { storm-super-resolution; } \\
\text { RRID:SCR_018302 }\end{array}$ \\
\hline ChimeraX software & Goddard et al., 2018 & $\begin{array}{l}\text { https://www.rbvi.ucsf.edu/chimerax/; } \\
\text { RRID:SCR_015872 }\end{array}$ \\
\hline ThunderSTORM & Jimenez et al., 2020 & $\begin{array}{l}\text { https://github.com/zitmen/thunderstorm; } \\
\text { RRID:SCR_016897 }\end{array}$ \\
\hline
\end{tabular}

\section{RESOURCE AVAILABILITY}

\section{Lead contact}

Further information and requests for resources and reagents should be directed to and will be fulfilled by the lead contact, Subhojit Roy (sroy@ucsd.edu).

Materials availability statement

Plasmids generated in this study are available from the Lead Contact without restriction.

Data and code availability

The MUDPIT-MS data are available in the supplemental information. Microscopy data reported in this paper will be shared by the lead contact upon request.

This paper does not report original code.

Any additional information required to reanalyze the data reported in this paper is available from the lead contact upon request.

\section{EXPERIMENTAL MODEL AND SUBJECT DETAILS}

Animals

CD1 pups obtained from Charles River Laboratories (Cat\#022-CD1) were used for preparing primary hippocampal cultures. Wistar rats were obtained from Janvier labs (France).

Primary cell cultures

For most experiments, hippocampal cultures were obtained from brains of postnatal (P0-P1) CD-1 mice and plated on MatTek glassbottom dishes as described previously in detailed published protocols (Ganguly and Roy, 2014; Roy et al., 2011), in accordance with University of California guidelines. Briefly, MatTek dishes were coated with $100 \mu \mathrm{l}$ of $1 \mathrm{mg} / \mathrm{mL}$ poly-d-lysine for $2 \mathrm{~h}$ at RT, washed thrice with $\mathrm{ddH}_{2} \mathrm{O}$, and air-dried before plating. Hippocampi from P0-P1pups were dissected in ice-cold dissection buffer (HBSS, $4.44 \mathrm{mM}$ d-glucose, and $6.98 \mathrm{mM}$ HEPES) and incubated in $0.25 \%$ Trypsin-EDTA at $37^{\circ} \mathrm{C}$ for 15 min. Following this, neurons were dissociated in plating media (10\% FBS and 90\% Neurobasal/B27; Life Technologies) by trituration. Neurons were plated at a density of 50,000 cells/100 $\mu \mathrm{l}$ (for FM4-64 experiments and clathrin imaging at en-passant boutons) and at 25,000 cells/100 $\mu$ l (for all other experiments) of plating media. Neurons were maintained in Neurobasal/B27 media (supplemented with $2 \%$ B27 and $1 \%$ GlutaMAX) in an incubator at $37^{\circ} \mathrm{C}$ and $5 \% \mathrm{CO}_{2}$ for $7-9 \mathrm{~d}$ before transfection. All the experiments on neuronal cultures were performed between 8-21 DIV. E18 rat hippocampal cultures were used for the DNA-PAINT experiments following standard protocols, and guidelines established by the European Animal Care and Use Committee (86/609/CEE) and local ethics committee (agreement D13-055-8) were followed.

\section{METHOD DETAILS}

DNA constructs, pharmacologic agents, neuronal cultures, transfections, and immunocytochemistry

The GFP:CLC (Gaidarov et al., 1999), pUC57-APEX (Martell et al., 2012) and Dendra2 constructs were obtained from Addgene [from the laboratories of Drs. James H. Keen (Thomas Jefferson University, PA), Alice Ting (Stanford University, CA) and Michael Davidson (Florida State University, Tallahassee, FL), respectively]. The PAGFP:CLC construct was subcloned from the PAGFP backbone (Patterson and Lippincott-Schwartz, 2002), a gift from Dr. George Patterson (NIH/NIBIB) and Jennifer Lippincott-Schwartz (Janelia Farms Research Campus, Ashburn VA), by standard cloning. CHC-T7-Hub and T7-empty backbone (Liu et al., 1998) was a gift from Dr. Frances Brodsky (UCL, London, UK). The GFP:Utr-CH (Burkel et al., 2007), and synaptophysin:mCherry constructs (Gerrow et al., 
2006) were gifts from Drs. William Bement (University of Wisconsin, Madison, WI) and Leon Lagnado (University of Sussex, Sussex, England, UK) respectively. pBa-Flag-BicD2-FKBP and pBa- Flag-tdTM-BicD2-FKBP constructs were from Gary Banker (Oregon Health \& Science University, Portland, OR). FRB-GFP:CLC was generated by inserting GFP:CLC in pFRB-3myc-Rab5a plasmid by replacing Rab5a (Bentley et al., 2015). GFP:synapsin-1a (Gitler et al., 2004) was a gift from George Augustine (Nanyang University, Singapore). EGFP-AP180 (Bushlin et al., 2008), GAK-GFP (Massol et al., 2006) and pEGFP-C1-Dynamin (Song et al., 2004) were kindly gifted by Drs. Pamela Yao (National Institute on Aging-National Institutes of Health, Baltimore, Maryland), Tomas Kirchhausen Harvard Medical School, Boston, MA and Sandra Schmid (University of Texas Southwestern Medical Center, Dallas, Texas) respectively. Dynasore, Latrunculin A (Life Technologies) and nocodazole were dissolved in DMSO and used at a final concentration of $80 \mu \mathrm{M}, 100 \mathrm{~nm}$ and $10 \mu \mathrm{g} / \mathrm{mL}$ respectively. Swinholide A and Rapamycin analog A/C heterodimerizer (Takara) were dissolved in ethanol and used at a final concentration of $100 \mathrm{nM}$ respectively. The stocks of FM4-64 (Invitrogen), Advasep-7, Live-or-Dye NucFix Red (Biotium) were prepared in DMSO and used at a final concentration of $10 \mu \mathrm{M}, 1 \mathrm{mM}$ and $0.1 \mathrm{x}$ in PBS (manufacturers' protocol) respectively. The glutamate receptor antagonists 6-cyano-7-nitroquinoxaline-2,3-dione (CNQX, TOCRIS bioscience), and D,L-2amino-5-phosphonovaleric acid (AP5, TOCRIS bioscience) were used at $10 \mu \mathrm{M}$ and $50 \mu \mathrm{M}$ concentration respectively. Unless otherwise noted, all drugs were purchased from Millipore-Sigma and added to Hibernate E media (see below) prior to imaging. The Hsc70D10N and control Hsc70 constructs were from N. Lamarche-Vane (McGill University, Montreal, Canada). The Hsc70 inhibitor VER155008 (Cat\#3803; Tocris Biosciences) was dissolved in DMSO and used at a working concentration of $100 \mu \mathrm{M}$.

Neurons were transfected with the indicated fluorescent constructs ( $0.8 \mu \mathrm{g}$ DNA for clathrin constructs, $1.2 \mu \mathrm{g}$ of all others) at 7-9 DIV with either Lipofectamine 2000 (Life Technologies) or Calcium phosphate transfection kit (Promega) (Sharma et al., 2019). Live imaging of axons was performed between 8-10 DIV, and boutons were imaged between 13-21 DIV. As dictated by the experiment, neurons were co-transfected with soluble-GFP/soluble -mCherry/synaptophysin:DsRed to identify axons/dendrites/boutons. GFP:CLC transfected neurons selected for imaging had punctate clathrin appearance in dendrites, and axon regions imaged were more than $150 \mu \mathrm{m}$ from the neuronal cell body. Boutons were selected $>150 \mu \mathrm{m}$ away from the soma on the basis of varicosities in the intensity along the axon and other morphological features typical of synapses (Wang et al., 2014).

For immunostaining, neurons were fixed in 4\% paraformaldehyde/sucrose solution in 1x PBS for 10 min at room temperature, followed by extraction in $0.2 \%$ Triton X-100 for $10 \mathrm{~min}$, and blocking in $1 \%$ bovine serum albumin/5\% FBS for $2 \mathrm{~h}$ at room temperature. To label endogenous clathrin, neurons were incubated overnight at $4 \circ \mathrm{C}$ with rabbit polyclonal antibody against clathrin heavy chain at 1:500 dilution. After washing the primary antibody with 1x PBS, neurons were blocked again for 30 min at room temperature, and incubated in goat-rabbit Alexa Fluor 594 (1:500) for $1 \mathrm{~h}$. for some experiments, GFP:CLC transfected neurons was stained with primary chicken polyclonal anti-GFP antibody (1:500) and secondary Alexa Fluor 488 (1:500). All primary and secondary antibodies were purchased from Abcam.

Live imaging, photobleaching, photoactivation, and photoconversion experiments

Most single-color live imaging experiments for axonal trafficking were performed on an Olympus IX81 inverted motorized epifluorescence microscope equipped with a CooISNAP HQ2 camera (Photometrics). Two-color live imaging was done either by simultaneous imaging of green/red channels using the Dual Cam imaging device (Photometrics) attached to two CoolSNAP HQ2 cameras (Olympus IX81), or by rapid sequential excitation of green/red LEDs (SPECTRA-X) on an inverted epifluorescence microscope (Eclipse Ti-E; Nikon) equipped with CFI Plan Apochromat VC $100 \times$ oil (NA 1.40; Nikon) objective, and an electron-multiplying charge-coupled device camera (QuantEM:512SC; Photometrics). A multi-band-pass filter (Chroma Technology Corp.) was inserted into the emission light path, and GFP/RFP images were obtained with precise subpixel registration. Before live imaging, neurons were transferred to Hibernate media (Brainbits), supplemented with 2\% B27, 2mM Glutamax, 0.4\% D-glucose, $37.5 \mathrm{mM} \mathrm{NaCl}$ (Ganguly et al., 2017; Roy et al., 2011; Scott et al., 2011), and maintained at $35.5^{\circ} \mathrm{C}$ to $37^{\circ} \mathrm{C}$ for the duration of the experiments (Precision Control, Weatherstation or a heated stage chamber, model STEV; World Precision Instrument, Inc.). Axons were identified by morphology, and only neurons with unambiguous morphology were selected for imaging (Roy et al., 2011; Scott et al., 2011). For experiments in Figure 1, GFP:CLC, neurons were either imaged every 0.7 s for several minutes, or PAGFP:CLC was photoactivated with $405 \mathrm{~nm}$ LED light for $1 \mathrm{~s}$, and imaged every $1.2 \mathrm{~s}$. Dendra2:CLC was photoconverted by $405 \mathrm{~nm}$ LED light for $3 \mathrm{~s}$, and imaged every $1.2 \mathrm{~s}$. Synaptophysin:GFP was imaged using the stream acquisition function (MetaMorph) at 5 frames/s (with no time interval between images). For imaging en passant boutons, 13-21 DIV neurons were co-transfected with synaptophysin:dsRed/sol-mCherry and GFP:CLC/GFP:Synapsin, and boutons were identified based on size of the synaptic vesicle cluster and morphology (Wang et al., 2014). Boutons along the primary axon, $>150 \mu \mathrm{m}$ from the soma and $<7 \mathrm{um}$ apart from the each other were selected for imaging. Additionally, for the GFP:CLC live imaging of en-passant boutons and intervening axonal-segments, boutons expressing $>2$ clathrin packets were selected and imaged at every $0.2 \mathrm{~s}$ (rapid imaging) for several minutes.

The clathrin photoactivation and photoconversion experiments in Figure 1 were done using a setup that has been described previously in detail (Roy et al., 2011). All other photobleaching and photoconversion experiments were done on the Nikon set-up described above, using Andor Mosaic3 Digital Micromirror Device (DMD) attached to a $405 \mathrm{~nm}$ diode laser (450 mW). All synaptic photobleaching and photoconversion experiments were done on DIV-13-14 neurons - a time when synapses are established in our post-natal cultures. For photobleaching, en passant boutons were bleached using the $405 \mathrm{~nm}$ laser ( 5 consecutive pulses of $1.5 \mathrm{~s}$ each), and imaged at every $2 \mathrm{~s}$ for several minutes. For photoconversion, Dendra2:CLC at boutons was photoconverted by pulsing the $405 \mathrm{~nm}$ laser for $1 \mathrm{~s}$, and boutons were imaged at $2 \mathrm{~s}$ time-intervals for several minutes. For the single clathrin packet 


\section{CellPress}

experiments, boutons were imaged every $1 \mathrm{~s}$ for several minutes. All movies were analyzed using kymographs generated with a builtin function in MetaMorph (Molecular Devices, LLC). Photoactivation and photoconversion data was analyzed using the intensity-center/bin-center shift method described previously in several publications from our group (Chakrabarty et al., 2019; Ganguly et al., 2017; Roy et al., 2011; Scott et al., 2011; Tang et al., 2013). All statistical analysis was performed using Graph Pad Prism (Graph Pad Software, San Diego, CA).

Rapamycin-inducible FKBP-FRB dimerizer and FM4-64 assays

Primary hippocampal neurons were co-transfected with the FRB-GFP:CLC and FKBP-BicD2 (+/- Tdtomato-tagged) constructs. Experiments were performed on 9-15 DIV neurons. Live neurons were incubated with 100 nM Rapalog for indicated times. Subsequently, neurons were either used for FM4-64 uptake assay or fixed for immunocytochemistry. Viability of neurons was evaluated using dead cell stain (Live-or-Dye NucFix Red), following the manufacturers' protocol. In brief, after the FKBP-FRB experiment, live neurons were incubated with the stain $\left(0.1 \mathrm{x}\right.$ in PBS) for $10 \mathrm{~min}$ at $37^{\circ} \mathrm{C}$. Thereafter, neurons were washed with PBS and imaged in HELF media at $37^{\circ} \mathrm{C}$. For positive controls, neurons were treated with $0.01 \%$ Triton for 1 min before staining. For the FM4-64 uptake assay, we used a protocol specifically optimized to evaluate CME (Cousin et al., 2018). Briefly, neurons were washed with imaging buffer $(2.5 \mathrm{mM} \mathrm{KCl}, 136 \mathrm{mM} \mathrm{NaCl}, 1.3 \mathrm{mM} \mathrm{MgCl} 2,2 \mathrm{mM} \mathrm{CaCl} 2,10 \mathrm{mM}$ glucose, $10 \mathrm{mM}$ HEPES) followed by $30 \mathrm{~s}$ incubation in high potassium stimulation buffer ( $50 \mathrm{mM} \mathrm{KCl}, 86 \mathrm{mM} \mathrm{NaCl}, 1.3 \mathrm{mM} \mathrm{MgCl} 2,2 \mathrm{mM} \mathrm{CaCl} 2,10 \mathrm{mM}$ glucose, $10 \mathrm{mM}$ HEPES) containing $10 \mu \mathrm{M}$ FM4-64. Thereafter, neurons were washed with imaging buffer containing $10 \mu \mathrm{M}$ FM4-64 for $90 \mathrm{~s}$. Finally, neurons were washed with Imaging buffer containing $1 \mathrm{mM}$ Advasep-7 for 2 min and imaged. All experiments were done at room temperature, with $\mathrm{pH}$ of all buffers adjusted to 7.4, and supplemented with $10 \mu \mathrm{M} \mathrm{CNQX}, 50 \mu \mathrm{M}$ DAP-5. For some experiments, neurons were preincubated with $80 \mu \mathrm{M}$ Dynasore before FM4-64 uptake.

Biochemical fractionation, western blotting and immuno-precipitation from mouse sciatic nerves

In vivo biochemical assays were adapted from protocols described earlier by (Das et al., 2013; Ganguly et al., 2017; Scott et al., 2011; Tang et al., 2012). Briefly, sciatic nerves were dissected from 6-8-wk-old mouse CD1 (WT) mouse. 64 sciatic nerves were pooled for each round of immune-precipitation (IP) and crushed in liquid nitrogen using a motor pestle and then homogenized in nondenaturing buffer (1X IP buffer, Invitrogen Dynabeads Co-Immunoprecipitation Kit [ThermoFisher Scientific, USA]) using 18 G and 23 G needles in the presence of protease inhibitor cocktail (Sigma-Aldrich). The resulting homogenate was centrifuged at $1,000 \mathrm{~g}$ for $20 \mathrm{~min}$ at $4 \mathrm{pC}$ to obtain a nuclear pellet (P1) and a post-nuclear supernatant (S1). The S1 supernatant was then centrifuged at $10,200 \mathrm{~g}$ for $20 \mathrm{~min}$ at $4 \mathrm{pC}$ to obtain a crude synaptosomal fraction (P2) and synaptosome-depleted fraction (S2). IP was performed using the DynaBeads Co-Immunoprecipitation kit (14321D; Thermo Fisher Scientific). After centrifugation, the S2 fraction was divided equally and incubated with 10.5 mg anti-Clathrin-Heavy-Chain antibody (ab21679; Abcam, Cambridge MA) and anti-Mouse IgG (ab190475; Abcam, Cambridge MA) for overnight coupling with magnetic beads at $37^{\circ} \mathrm{C}$. All following washes were performed as per the manufacturer's protocol. The S2 fraction was incubated with the antibody-coupled beads for $35 \mathrm{~min}$ at $4^{\circ} \mathrm{C}$ on a rotor. After the final wash, $3 \mathrm{mg}$ beads from each fraction were subjected to $2 \mathrm{D}$ gel electrophoresis on a $4 \%-12 \%$ gradient SDS-page gel and then probed with the anti-Clathrin-Heavy-Chain antibody to determine the efficacy of immunoprecipitation. The remaining $7.5 \mathrm{mg}$ of $\mathrm{S} 2$ fraction lysate beads were then used for MudPIT-MS analysis. Two independent repeats were performed with sciatic nerve axons S2 lysate.

Protein identification through MudPIT-MS analysis and in silico data analysis

Overall steps in MudPIT-MS protocols were identical to our previous studies (Ganguly et al., 2017). Briefly, beads coated with the clathrin heavy chain antibody (or mouse lgG) were dissolved in $100 \mu \mathrm{L}$ of $8 \mathrm{M}$ urea in $100 \mathrm{mM}$ Tris, pH 8.5, followed by reduction and alkylation in $10 \mathrm{mM}$ Tris (2-carboxyethyl) phosphine hydrochloride (Sigma) and $55 \mathrm{mM}$ iodo-acetamide (Sigma-Aldrich), respectively. This was followed by digestion in trypsin (Promega; incubated at $37^{\circ} \mathrm{C}$ overnight in the dark) and magnetic bead removal by a magnetic separator. The resulting protein digest was acidified in formic acid followed by centrifugation at 14,000 rpm for 10 min. Thereafter, the supernatant was pressure loaded onto a $250 \mu \mathrm{m}$ inner diameter-fused silica capillary column (Polymicro Technologies). This column was fitted with a Kasil frit packed with $2.5 \mathrm{~cm}$ of $5 \mu \mathrm{m}$ Partisphere strong cation exchange resin (Whatman) and $2.5 \mathrm{~cm}$ of $5 \mu \mathrm{m} \mathrm{C18}$ resin (Phenomenex). After desalting, this biphasic column was connected to a $100 \mu \mathrm{m}$ inner diameter-fused silica capillary (Polymicro Technologies) analytical column with a $3 \mu \mathrm{m}$ pulled tip packed with $10 \mathrm{~cm}$ of $3 \mu \mathrm{m}$ C18 resin (Phenomenex). The entire three-phase column was then laced in line with a 1,200 quaternary HPLC pump (Agilent Technologies) and analyzed using a modified 12-step separation described previously (Washburn et al., 2001). As peptides were eluted from the microcapillary column, they were electrosprayed directly into a hybrid LTQ Orbitrap Velos mass spectrometer (Thermo Fisher Scientific). A cycle consisted of one full-scan mass spectrum (300-1,600 m/z) followed by 20 data-dependent collision-induced dissociation tandem MS spectra. The application of mass spectrometer scan functions and HPLC solvent gradients was controlled by the Xcalibur data system (Thermo Fisher Scientific). Tandem MS spectra were extracted using RawXtract (version 1.9.9; (McDonald et al., 2004) and searched with the ProLuCID algorithm (Xu et al., 2015) against a mouse UniProt database concatenated to a decoy database in which the sequence for each entry in the original database was reversed (Peng et al., 2004). The ProLuCID search was performed using semienzyme specificity and static modification of cysteine because of carboxyam-idomethylation (57.02146). ProLuCID search results were assembled and filtered using the DTA Select algorithm (version 2.0; (Tabb et al., 2002)). The protein identification false positive rate was kept below $1 \%$, and all peptide-spectra matches had $<10$ ppm mass error. 
For selection of peptides from the raw MudPIT data, peptides were subjected to the following selection criteria. First, the same peptide fragment had to appear in both rounds of the co-immunoprecipitation, followed by the criteria that the total raw spectrum count from both rounds for the peptide should be $>30$. Finally, only peptides with combined spectrum counts twenty-fold or higher than the combined spectrum counts from IgG coupled beads were selected. Only peptides that meet all three criteria were included in the final list (Table S1). The identification of peptides and gene names was performed using the Uniprot database (https://www. uniprot.org/). For generation of the clathrin protein interaction map from the S2 fraction all known interactions from experimental data were determined from the String protein interaction database (http://www.string-db.org).

\section{Sample preparation, data acquisition and data processing for electron tomography}

For EM, mouse hippocampal neurons were plated at a density of 25,000 cells $/ 100 \mu \mathrm{l}$ in wells of gridded MatTek dishes coated with poly-D-lysine. DIV 9-10 neurons were transfected with APEX:GFP:CLC using lipofectamine 2000 as described previously (Ganguly et al., 2017). 6-8 $\mathrm{h}$ before transfection, $7 \mu \mathrm{M}$ Hemin chloride (Sigma-Aldrich) was added to the culture medium and kept in the medium until the fixation step (below). 12-16 h after transfection, transfected neurons were identified based on the GFP signal, and corresponding grid numbers were noted. Following this, neurons were fixed for 5 min at room temperature, and then 60 min on ice in $2.5 \%$ glutaraldehyde in $0.1 \mathrm{M}$ sodium cacodylate buffer $(\mathrm{pH} 7.4)$. After washes in cacodylate buffer and a quenching step in $20 \mathrm{mM}$ glycine, cells were incubated with freshly prepared DAB $(25.24 \mathrm{mM})$ and $0.03 \% \mathrm{H}_{2} \mathrm{O}_{2}$ for $25-30$ min. Neurons were then washed in $0.1 \mathrm{M}$ cacodylate buffer and post-fixed with $1 \%$ osmium tetroxide for $30 \mathrm{~min}$ on ice. The neurons were washed with $\mathrm{ddH}_{2} \mathrm{O}$ and then dehydrated and embedded in Durcupan epoxy resin. After curing, the coverslip from the bottom of the MatTek dish was removed gently, and grid numbers with transfected neurons were sawed out and mounted on a dummy acrylic block for sectioning. Ultra-thin $(70 \mathrm{~nm})$ and semi-thin sections $(200 \mathrm{~nm})$ were cut using a diamond knife (Diatome). To improve stability of specimens under the beam of the EM for tomography, these sections were coated with carbon on both sides. Colloidal gold particles ( $5 \mathrm{~nm}$ and $10 \mathrm{~nm}$ diameter) were deposited on each side of the sections to serve as fiducial markers.

Ultra-thin sections were imaged on the JOEL $120 \mathrm{kV}$ while the EM tomogram data were obtained from distal axonal regions ( $>150 \mu \mathrm{m}$ from the cell body) of transfected neurons using a FEl Titan high base microscope operated at $300 \mathrm{kV}$ and micrographs were produced using a $4 \mathrm{~K} \times 4 \mathrm{~K}$ Gatan CCD camera (US4000). Both microscope and detector were controlled by the Serial EM software package (Mastronarde, 2005) which managed the automated tilt series acquisition. Technical details of how the sections of the tomogram were acquired, image series were aligned properly, and its 3D representations were created have been described by (Phan et al., 2017). Briefly, sections were tilted every $1 \mathrm{~b}$ from $-60^{\circ}$ to $+60^{\circ}$, aligned properly using colloidal gold particles as fiducial markers and final high-quality 3D representations were built from the projection sets using a custom written non-linear bundle adjustment scheme in TxBR (Lawrence et al., 2006). 3D tomograms were visualized in IMOD (Kremer et al., 1996) and only axon regions with clathrin organelles which do not open to the surface were selected for representative images. EM tomograms were performed on APEX-GFP:CLC transfected neurons from three separate cultures. At least 2-3 independent transfected neurons from each experiment were imaged. 8 axons and 9 dendrites were imaged in total, with tomograms for at least 3 ROls acquired for each transfected axon and dendrite. A total of 62 coated-pits were identified from tomograms of dendrites, and 17 clathrin coated structures were identified in tomograms from axons. For immuno-EM experiments, a mouse monoclonal antibody against clathrin (clone X22, 1:200-500; Affinity Bioreagents) was used. Detailed procedures including preparation, fixation and immunogold labeling are described in Tao-Cheng, 2020.

\section{DNA-PAINT imaging and image analysis}

Rat hippocampal neurons were cultured on $18 \mathrm{~mm}$ coverslips at a density of 6,000/cm from embryonic day 18 pups following established guidelines of the French Animal Care and Use Committee (French Law 2013-118 of 1st February 2013) and approval of the local ethics committee (agreement 2019041114431531-V2 \#20242). To selectively label identified axons, neurons in some experiments were transfected the day before fixation with actin-GFP using Lipofectamine 2000 according to the manufacturer's instructions. After 9 to 13 DIV, neurons were fixed at RT in phosphate buffer (PB) $0.1 \mathrm{M} \mathrm{pH} 7.3$ containing $4 \%$ paraformaldehyde and $4 \%$ sucrose for $10 \mathrm{~min}$, followed by permeabilization and blocking in incubation buffer (IB): $0.22 \%$ gelatin, $0.1 \%$ Triton in PB $0.1 \mathrm{M} \mathrm{pH}$ 7.3 at RT for $2 \mathrm{~h}$, and incubated overnight at $4{ }^{\circ} \mathrm{C}$ with primary antibody in IB: polyclonal chicken anti-Map2 (Abcam \#53392, 1:1000), polyclonal rabbit anti-clathrin heavy chain (Abcam \#21679, 1:150) and monoclonal mouse anti $\beta 2$-spectrin (for axon shaft images, BD Bioscience 612563, 1:150) or monoclonal mouse anti-synapsin (Synaptic Systems \#106-001, 1:500. After rinses, neurons were incubated with secondary antibodies, including anti-rabbit D2 and anti-mouse D2 DNA-coupled secondary antibodies for DNA-PAINT (Ultivue) at RT for $1 \mathrm{~h}$.

DNA-PAINT imaging (Jungmann et al., 2014) was performed on an N-STORM microscope (Nikon Instruments) in imaging buffer with 0.25-1 nM Imager-650 and nM Imager-560 (Ultivue). The sample was alternatively illuminated at $647 \mathrm{~nm}$ and $561 \mathrm{~nm}$ (full laser power) and 20,000-30,000 images of each channel were acquired at 25-33 Hz. DNA-PAINT acquisitions were processed using the NSTORM software followed by post-processing; filtering and image reconstruction at 4 or $12 \mathrm{~nm} /$ pixel with the ThunderSTORM Fiji plugin (Jimenez et al., 2020). For 3D rendering, localizations were density-filtered ( $50 \mathrm{~nm}$ 3D radius) before reconstruction of individual presynapses as of 4-nm voxel stacks. Stacks where then 3D Gaussian-smoothened and imported into ChimeraX software (Goddard et al., 2018). After surface thresholding and smoothing (default values), 3D rendering images and movies were exported as images and movies. Clathrin particles were segmented using the Segger plugin and their volume measured. Volume $\mathrm{V}$ in $\mu \mathrm{m} 3 \mathrm{was}$ converted 
in equivalent sphere diameter $D$ in $n m$ using $\left[D=1000^{*} 2^{\star} V(\wedge 1 / 3)^{\star} 3 /\left(4^{*} \pi\right)\right]$ for graphing. For presynaptic clathrin particles, only particles contacting or apposed to the synapsin cluster were counted and measured.

\section{QUANTIFICATION AND STATISTICAL ANALYSIS}

\section{FRAP Quantification}

FRAP data were analyzed as previously described (Applewhite et al., 2007; Boyer et al., 2020). Background fluorescence values were subtracted from all data at each time point, and photobleaching was estimated by fitting an exponential decay function $\left(F=F_{O} \times e^{-k t}\right.$, where $F$ is fluorescence, $F_{O}$ is initial fluorescence, $k$ is the decay time constant, and $t$ is time) to the fluorescence of an unbleached control region in the same image. Fluorescence curves were normalized between cells by subtracting the fluorescence immediately after bleaching (time $=0$ ), and then dividing by the average of the 5 pre-bleach fluorescence time points. Fluorescence recovery halftime and immobile fraction were calculated from an inverse exponential decay $\left(F=A \times\left(1-e^{-\tau t}\right)\right.$, where $F$ is fluorescence, $A$ is the recovery plateau fluorescence, $\tau$ is the recovery time constant, and $t$ is time) fit to the average fluorescence recovery curve for each protein or treatment. Photobleaching curves were fit to the data using the Solver add-in of Microsoft Excel 2013, and fluorescence recovery curves were fit using GraphPad Prism.

\section{Statistical Analyses}

Datasets were tested for normality using the Shapiro-Wilk W test with $\alpha$ set at 0.05 . Pairwise comparisons were made using a Student's t test only when both groups being compared were found to be normally distributed: in all other cases, a Mann-Whitney nonparametric $U$ test was used. Correlation of clathrin to synapsin volume at synapses was performed using a Pearson's test. All three-group comparisons contained non-normally distributed data; therefore, nonparametric Kruskal-Wallis tests were used for all three-group comparisons. Post hoc pairwise comparisons were performed with Mann-Whitney nonparametric $U$ tests and were corrected for multiple comparisons using the Benjamini-Krieger-Yekutieli two-stage step-up method. No pre-hoc sample size estimates were determined prior to experiments. Details of experimental design are described in the experiment specific subsections of Method details section. All the data are presented as mean \pm SEM. The values of $n$ and what $n$ represents are included in the legends of the respective figures. 
Neuron, Volume 109

Supplemental information

Clathrin packets move in slow axonal transport

and deliver functional payloads to synapses

Archan Ganguly, Rohan Sharma, Nicholas P. Boyer, Florian Wernert, Sébastien Phan, Daniela Boassa, Leonardo Parra, Utpal Das, Ghislaine Caillol, Xuemei Han, John R. Yates III, Mark H. Ellisman, Christophe Leterrier, and Subhojit Roy 
- 8 supp figs

- 1 supp table

Supp Fig. 1 is related to main Fig. 1 Supp Fig. 2 is related to main Fig. 2 Supp Fig. 3 is related to main Fig. 3 Supp Fig. 4 is related to main Fig. 4 Supp Fig. 5 is related to main Fig. 5 Supp Fig. 6 is related to main Fig. 6 Supp Fig. 7 is related to main Fig. 7 Supp Fig. 8 is related to main Fig. 8 
Figure S1, related to Figure 1

A.

GFP:CLC in axons (example 1)

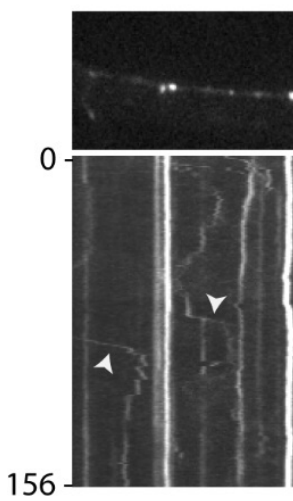

\section{GFP:CLCin axons (example 1)}

B.

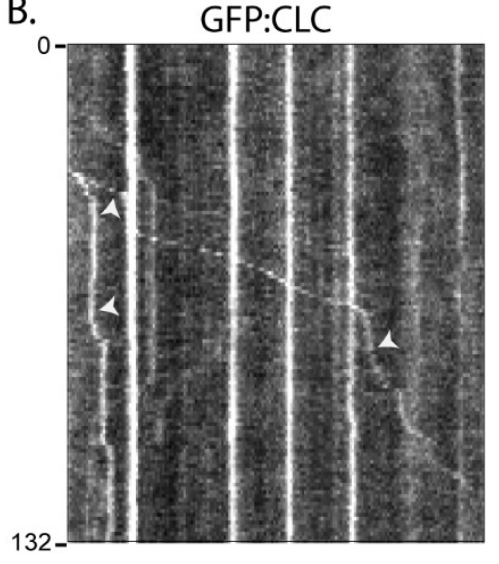

Synaptophysin:mCh

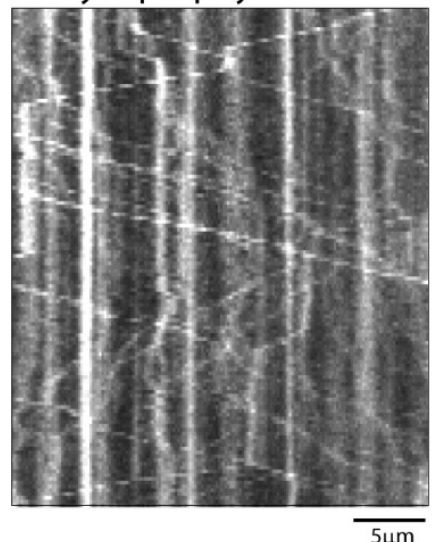

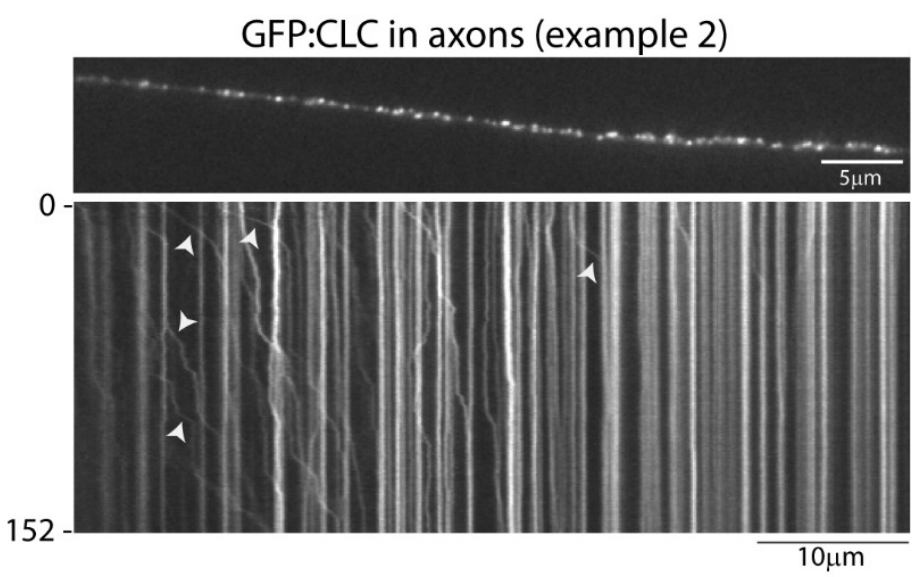

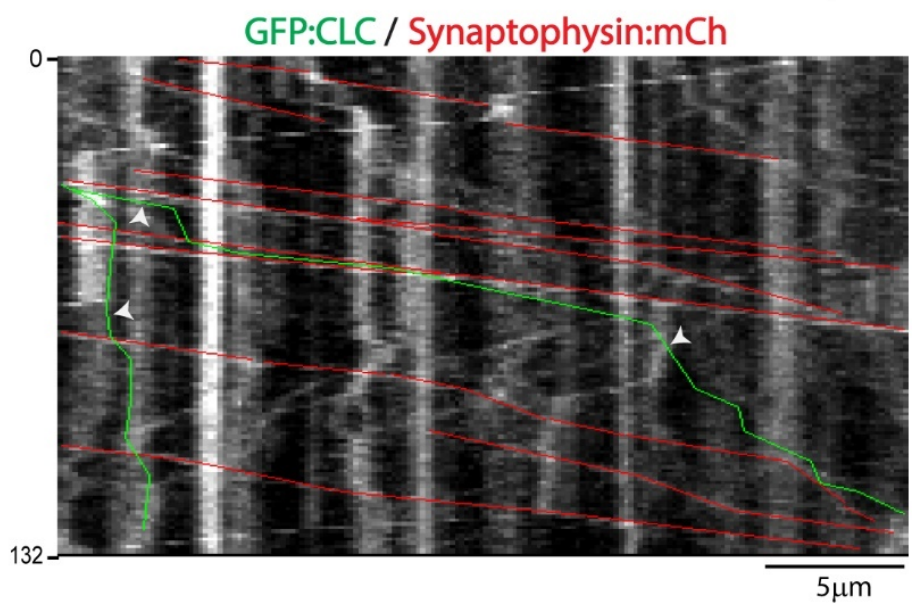

\section{Figure S1: Axonal clathrin dynamics.}

(A) Two examples of GFP:CLC dynamics in axons (image of axon on top, kymograph at bottom). Unlike dendrites, blinking (on/off) dynamics are rare in axons, and a fraction of particles move intermittently (arrowheads). Example 1 shows an axon with lower density of clathrin particles and example 2 shows an axon with higher particle density. Note that stationary and moving particles are seen in both cases.

(B) Kymographs from dual-imaging of GFP:CLC and synaptophysin:mCherry (left), with overlaid tracks (right). The movement of clathrin transport-packets was much less frequent than the fast transport of synaptophysin-vesicles. Though the extensive movement of synaptophysin made definitive conclusions difficult, we could see clear instances where clathrin-packets were transported independently of synaptophysin - see overlaid clathrin (green) and synaptophysin (red) tracks in kymograph below.

Time in seconds to the left of kymographs, and scale bar on lower right. 


\section{Figure S2, related to Figure 2}

A. Alexa-TfR uptake in cultured neurons DsRed only (control)

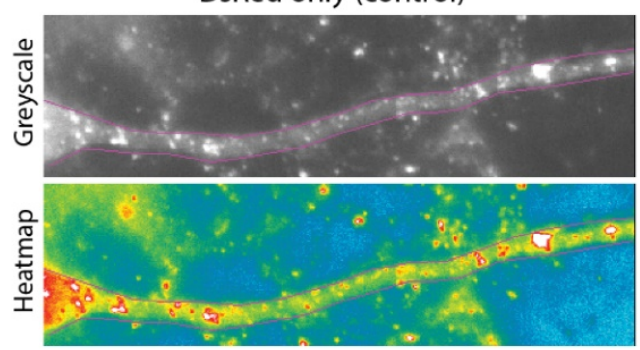

C.

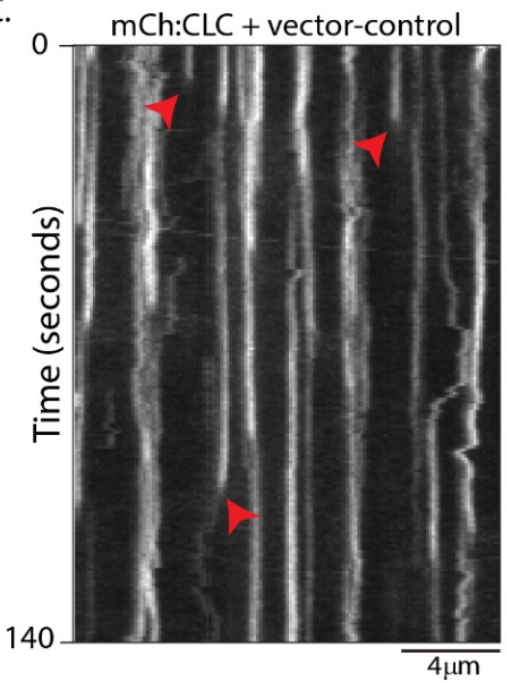

$\mathrm{mCh}: \mathrm{CLC}+\mathrm{T} 7-\mathrm{Hub}$ mutant

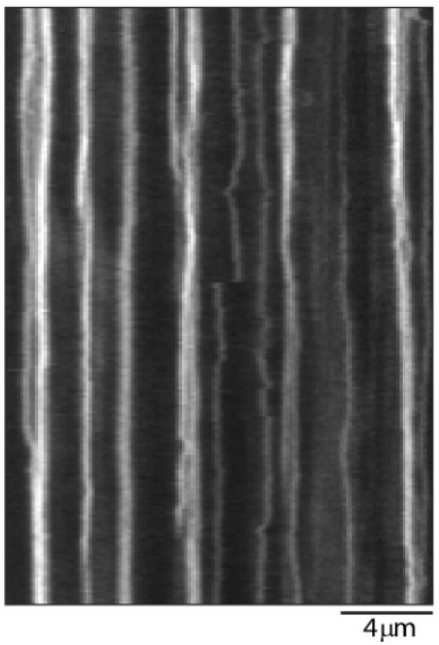

B.

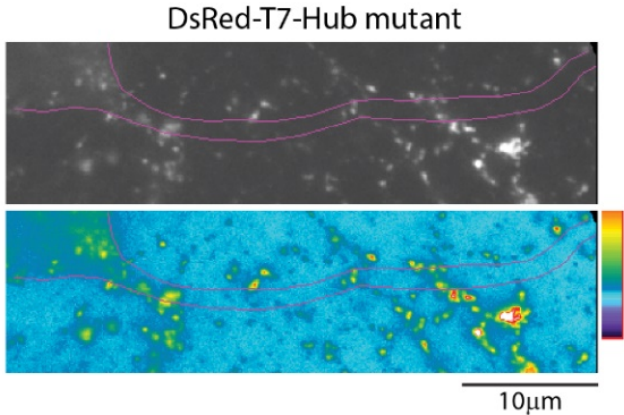

D.

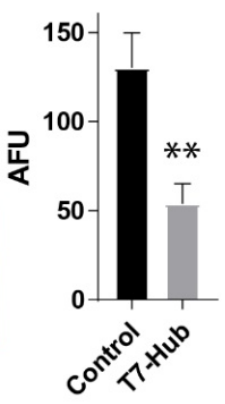

mCherry-CLC + vector backbone $(\mathrm{n}=6)$
mCherry-CLC + CHC-T7-Hub $(\mathrm{n}=6)$

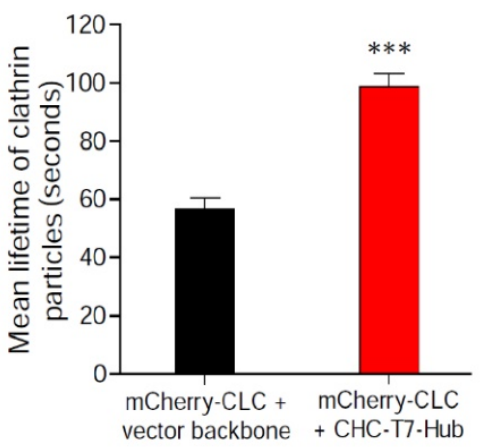

E. Effect of Nocodazole on vesicle transport

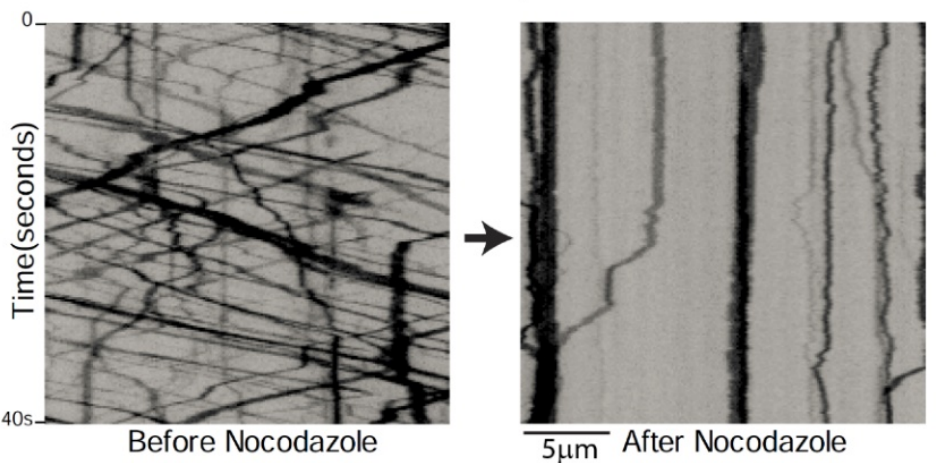

Figure S2: The clathrin T7-Hub mutant blocks receptor mediated endocytosis.

(A) Neurons were transfected with T7-Hub mutant (DsRed-tagged) or vector-control (DsRed-only), and Alexa-488-transferrin uptake into the transfected neurons was analyzed. As evident in the representative greyscale images (above) and pseudocolor heatmaps (below), the T7-Hub mutant attenuates transferrinuptake. Data quantified in (B), 5-6 neurons per condition, from one culture; ${ }^{* *} p<0.001$.

(C) Kymographs showing dynamics of mCh:CLC in dendrites co-transfected with the T7-Hub mutant (or vector control). Note that the blinking of clathrin fluorescence - due to receptor mediated endocytosis, some marked by arrowheads - is essentially abolished in the T7-Hub transfected neurons.

(D) Quantification of mCh:CLC lifetime in dendrites (100-150 particles from 6 dendrites were analyzed; $\left.{ }^{* * *} p<0.0001\right)$.

(E) Kymographs showing movement of the pan-vesicle marker NPYss:mCherry (see Ganguly et al., 2015) in an axon before and after $10 \mu \mathrm{g} / \mathrm{ml}$ Nocodazole for $30 \mathrm{~min}$. Note complete stoppage of vesicle transport after adding the microtubule depolymerizing agent.

Time in seconds to the left of kymographs, and scale bar on lower right. 


\section{Figure S3, related to Figure 3}

A.
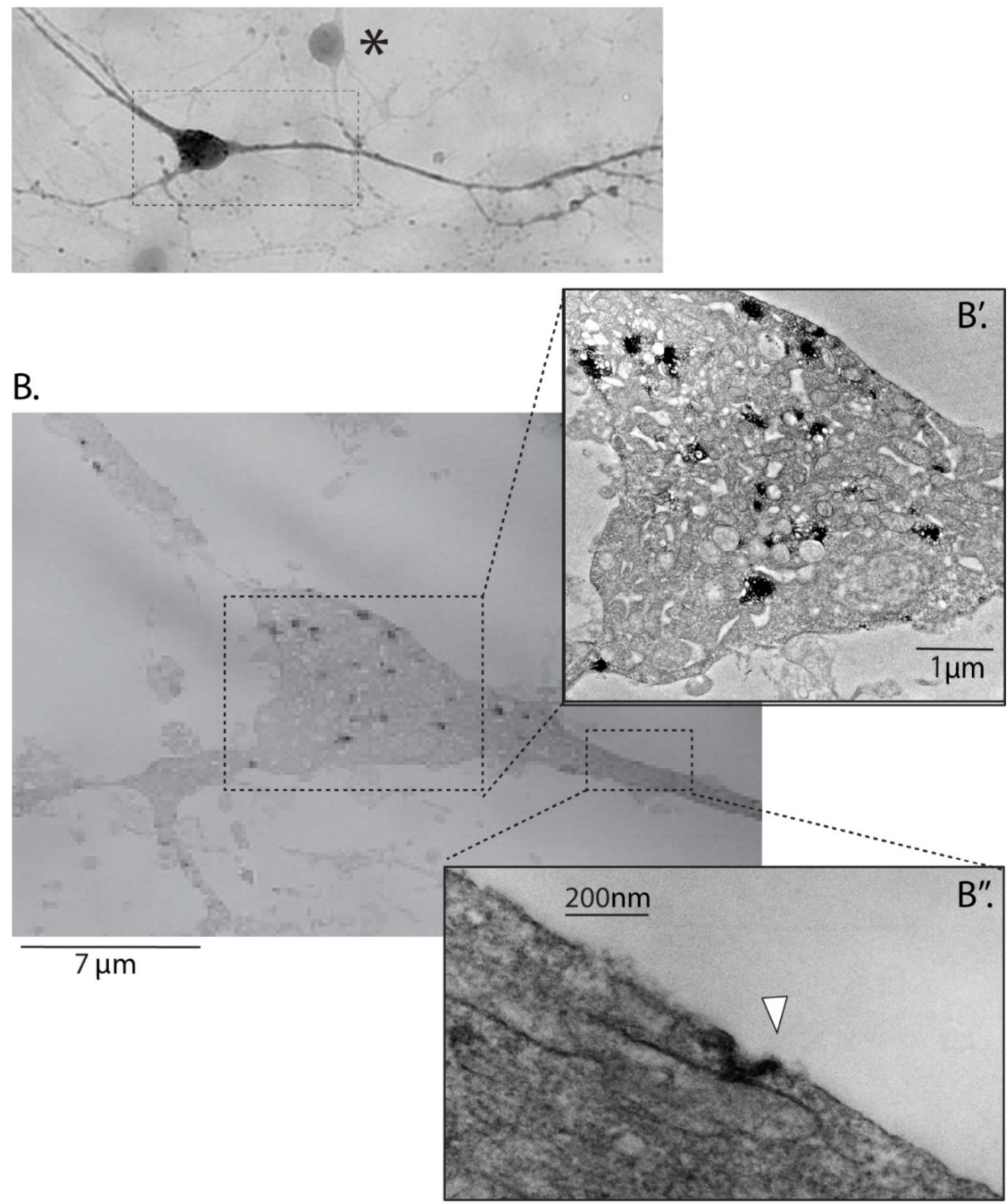

\section{Figure S3: Apex-clathrin labeling and EM.}

(A) Neurons were transfected with Apex:GFP:CLC and processed for EM. Gridded coverslips were used to identify transfected neurons for EM processing, as described in methods. The DAB signal from an Apex:GFP:CLC transfected neuron is shown, along with an adjacent un-transfected neuron (marked with an asterisk).

(B) EM of rectangular region from (A), dark signals represent clathrin. Note that many clathrin structures are seen in the somatodendritic region (B'), some clearly representing coated clathrin pits (B"). 
Figure S4, related to Figure 4

A. Fractionation and Co-IP/MudPIT-MS analysis

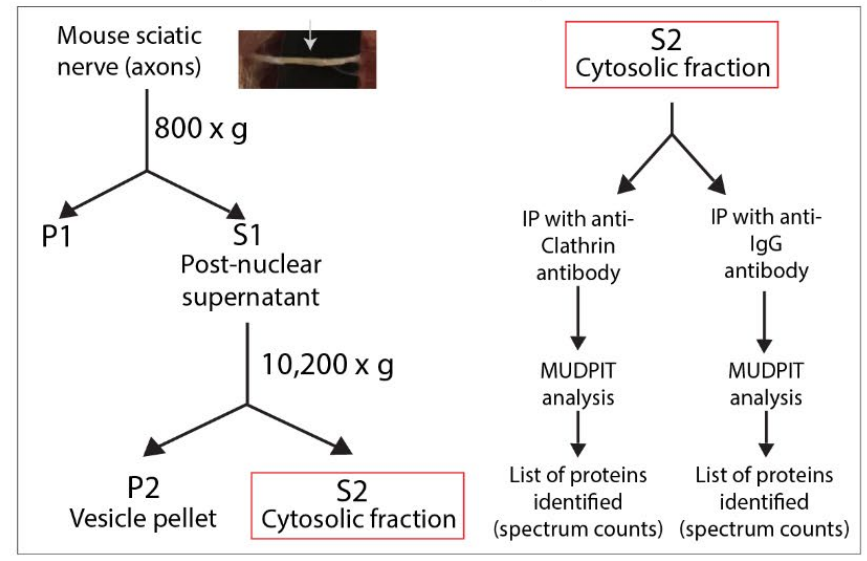

C. Dynamics of clathrin and GAK in dendrites
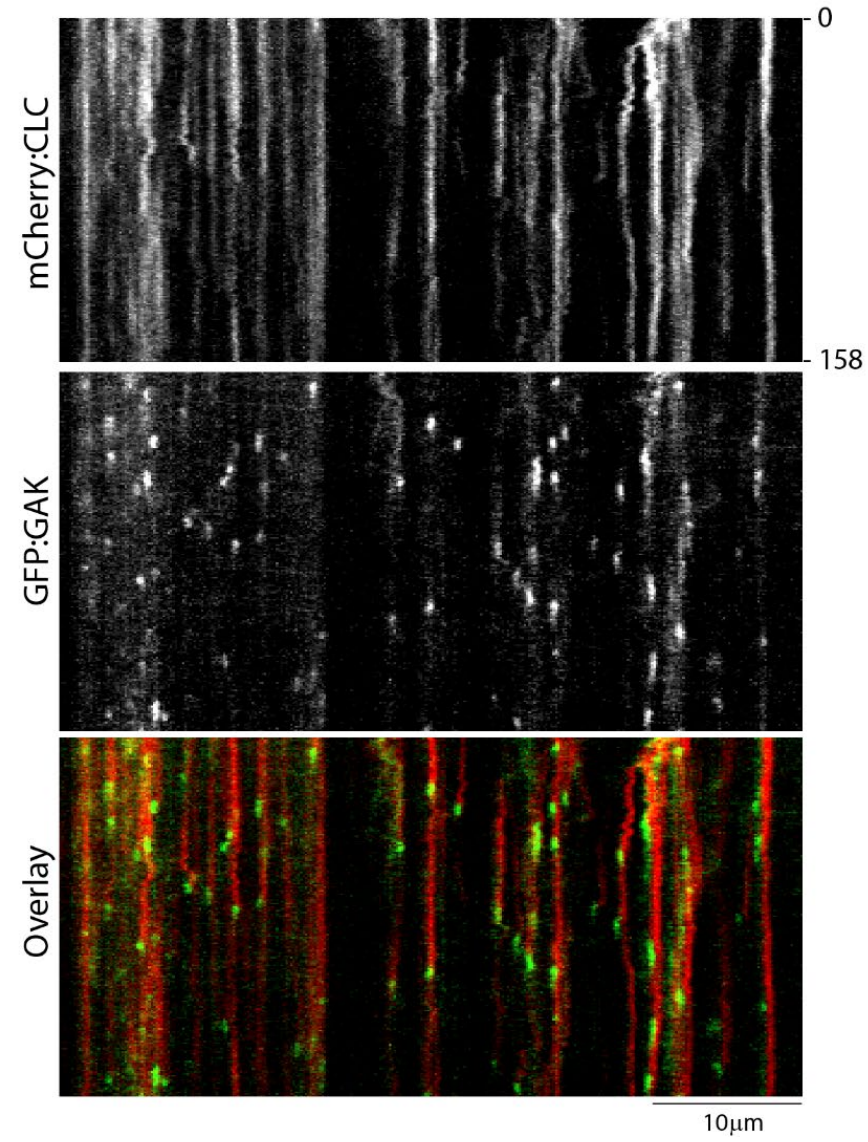

B.
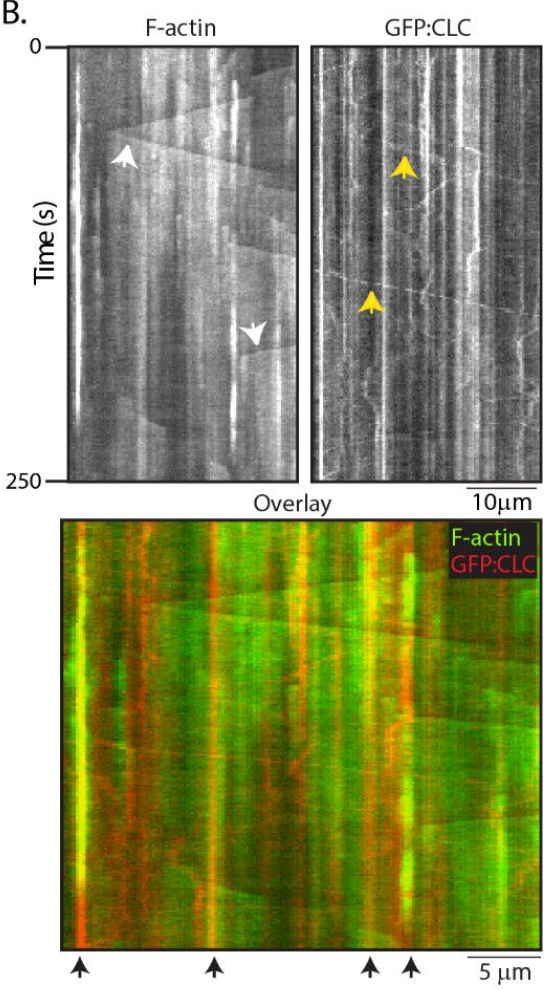

D. GFP:CLC motility after $\mathrm{Hsc70}$ inhibition
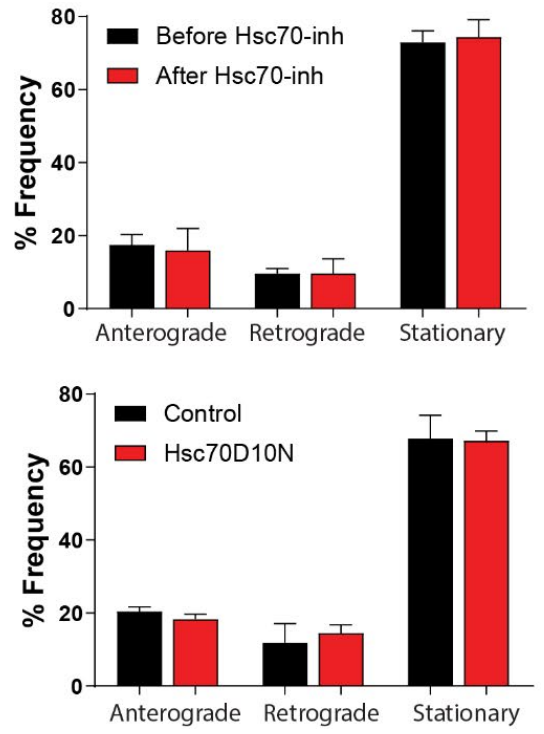

Figure S4: Composition of axonal clathrin packets.

(A) Schematic for fractionation and Mudpit-MS. Clathrin was immunoprecipitated from cytosolic (S2) sciatic nerve fractions (dissected from mice, see inset picture and arrow), and associating proteins were identified by MS.

(B) Kymographs from two-color imaging of GFP:Utr-CH (labeling F-actin) and mCh:CLC - some 'actin trails' and clathrin transport-packets are marked by white and yellow arrowheads respectively. On the overlaid red/green kymographs on right, note that many stationary clathrin particles are colocalized with F-actin (black arrowheads, bottom).

(C) Simultaneous two-color imaging of mCherry:CLC and GFP:GAK in dendrites. Note that GAK particles only appear in kymographs when clathrin is disassembled, consistent with the role of GAK as a protein that dis-assembles clathrin cages. Also note that this behavior of GAK is very different from its dynamics in axons, where many GAK particles colocalize with clathrin and are intermittently transported (Fig. 4D).

(D) Quantification of GFP:CLC particle dynamics in axons before and after pharmacologic inhibition of Hsc70 with a small-molecule (VER155008); or genetic interference of Hsc70 function using a dominant-negative construct (Hsc70D10N). Note that motility of clathrin is unchanged after Hsc70 inhibition. 


\section{Figure S5, related to Figure 5}

A. DNA-PAINT of clathrin at presynapses

$A^{\prime}$. Zoom from inset in (A), used for Fig. 5A
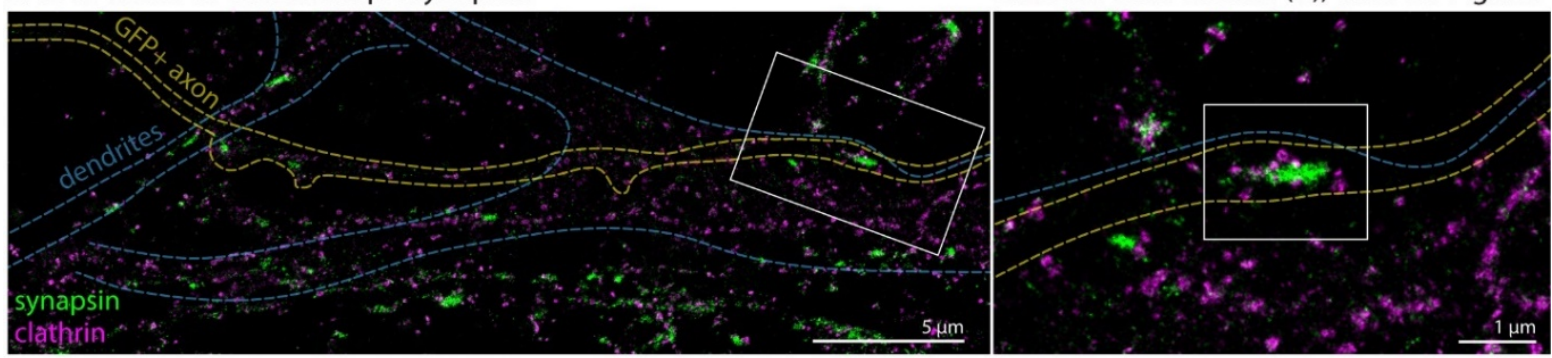

B. Immunogold EM of clathrin

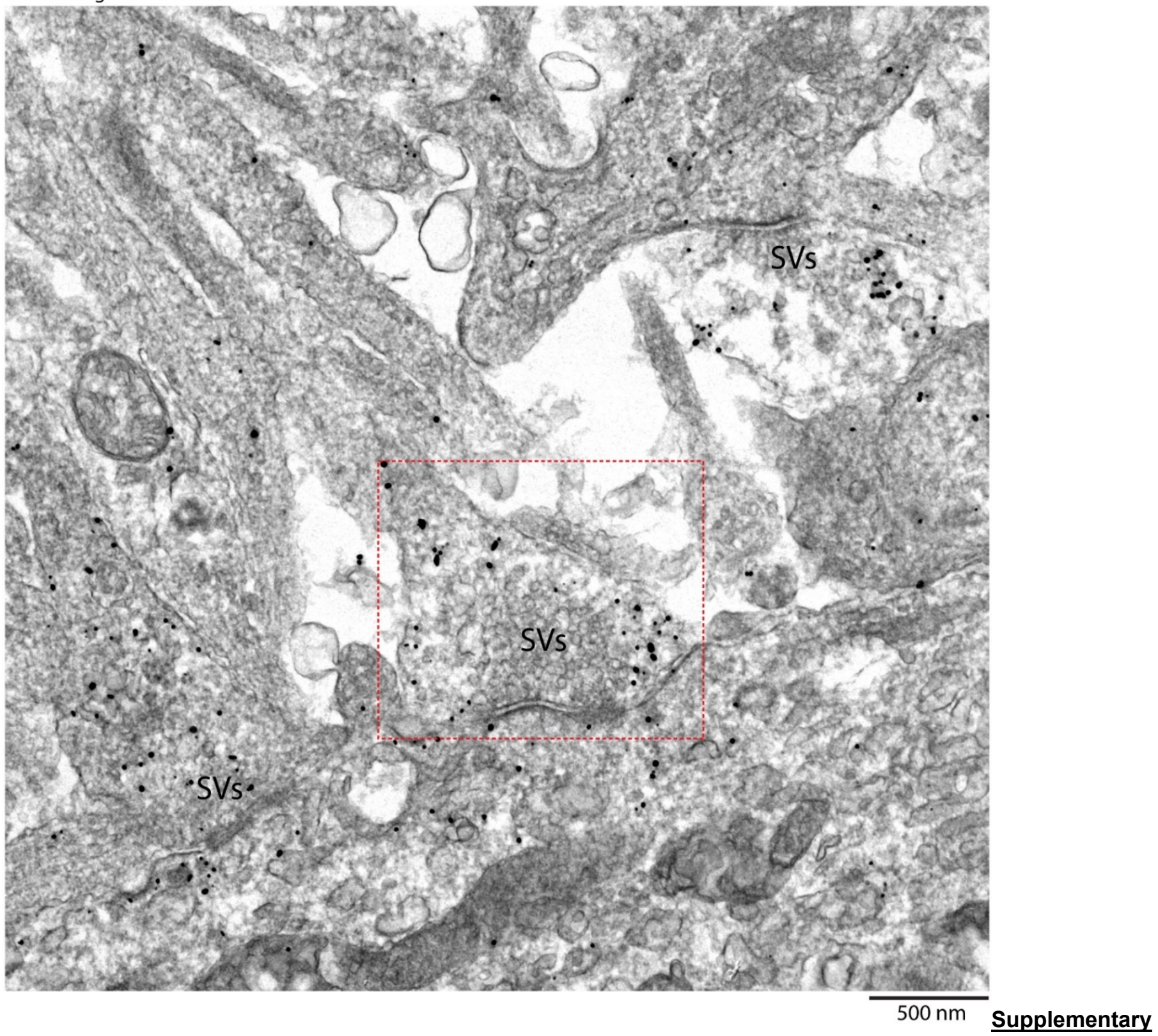

Figure S5: Supplements super-resolution and immuno-EM data shown in Fig. 5.

(A) DNA-PAINT: Larger field of view showing axons and dendrites corresponding to the bouton shown in Fig. 5A. Blue dashed lines represent dendrites (identified by MAP2 staining), and dashed yellow lines represent an axon (identified by Actin:GFP transfection). A' shows the actual image shown in Fig. 5A.

(B) Immuno-EM of clathrin - Large field of view showing synapses and neurites around the synapse showed in Fig. 5F1 (dashed red box marks ROI used in figure). Note that the immuno-gold particles do not bind to many other structures (tubulo-vesicular organelles, mitochondria, cytoskeletal structures, cytoplasmic matrix, etc.) and appears specific for clathrin. 


\section{Figure S6, related to Figure 6}

A. Trafficking of GFP:CLC at synapses

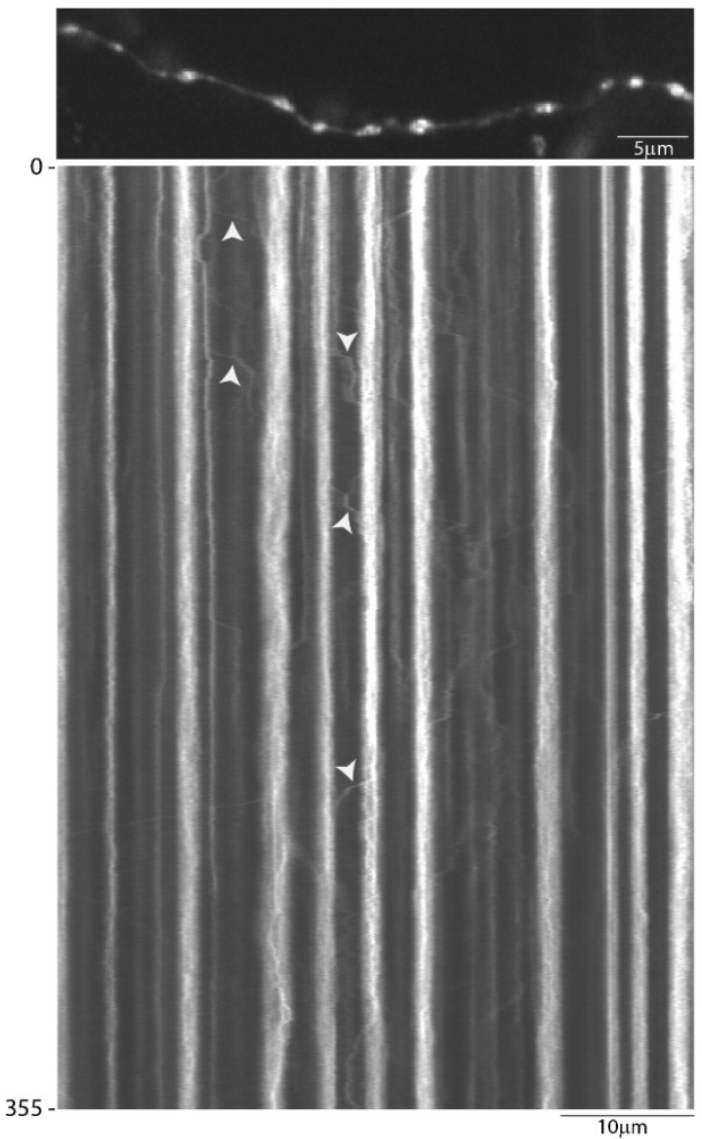

B. Principle of "pass/trap" analyses (GFP:CLC)
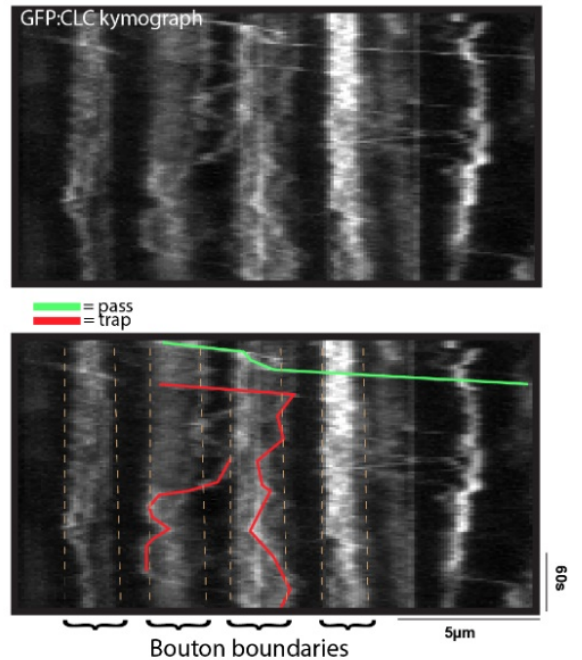

C. FRAP of synapsin

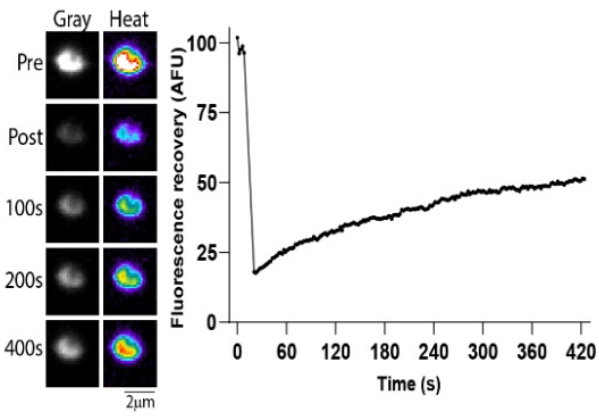

D. GFP:CLC FRAP at boutons (DIV 14-21)

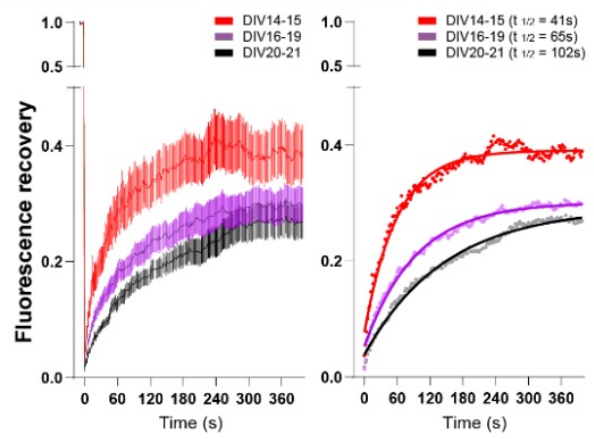

E. Distribution of endogenous clathrin intensities

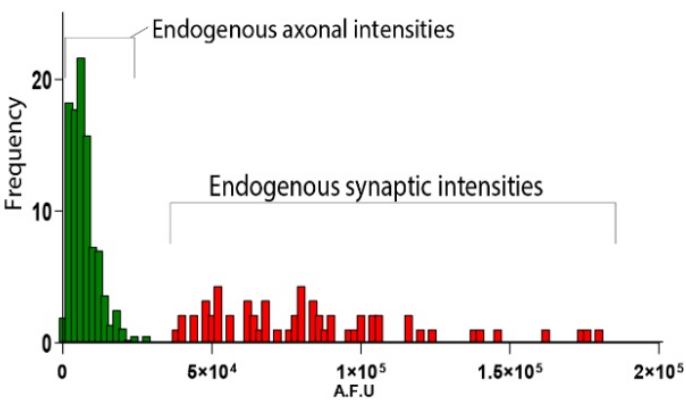

Figure S6: Trafficking and synaptic targeting of clathrin particles.

(A) Image (top) and kymograph (bottom) of GFP:CLC trafficking in presynaptic boutons (varicosities) and flanking axons. Note that many clathrin particles are exchanged between boutons. Elapsed time on left, and scale-bar on lower right of kymograph.

(B) Principle of "pass/trap analysis" to quantify moving clathrin particles trapped at boutons. Moving GFP:CLC (or Dendra2:CLC) particles that did not re-emerge from boutons after 60 seconds were considered trapped. Green and red lines show examples of passing and trapped GFP:CLC particles. Time/scalebar on lower right.

(C) Time-series grayscale and heatmap images of a bouton containing GFP:synapsin that was photobleached, with corresponding FRAP curve on right (time in seconds shown on left of images). Note the relatively smooth, gradual rise of GFP:synapsin fluorescence after photobleaching.

(D) FRAP-curves of GFP:CLC from boutons of neurons cultured for 14-21 days in vitro (DIV). Note that there is an earlier flattening of the FRAP-curves with increasing DIV - suggesting a larger immobile pool with age in cultures - likely reflecting synaptic maturity over time (data from 34 neurons, 5 separate cultures for DIV14-15; 51 neurons, 10 separate cultures for DIV16-19; and 44 neurons, 6 separate cultures for DIV20-21). All clathrin photobleaching and photoconversion experiments in main figures were done on DIV 13-14 neurons.

(E) Intensity distributions (histograms) of endogenous clathrin in axons and synapses, determined by immunostaining cultures with antibody to clathrin heavy chain (to mark synapses, see methods; 355 axonal particles and 92 synapses were analyzed from 2 separate cultures). 


\section{Figure 57 , related to Figure 7}
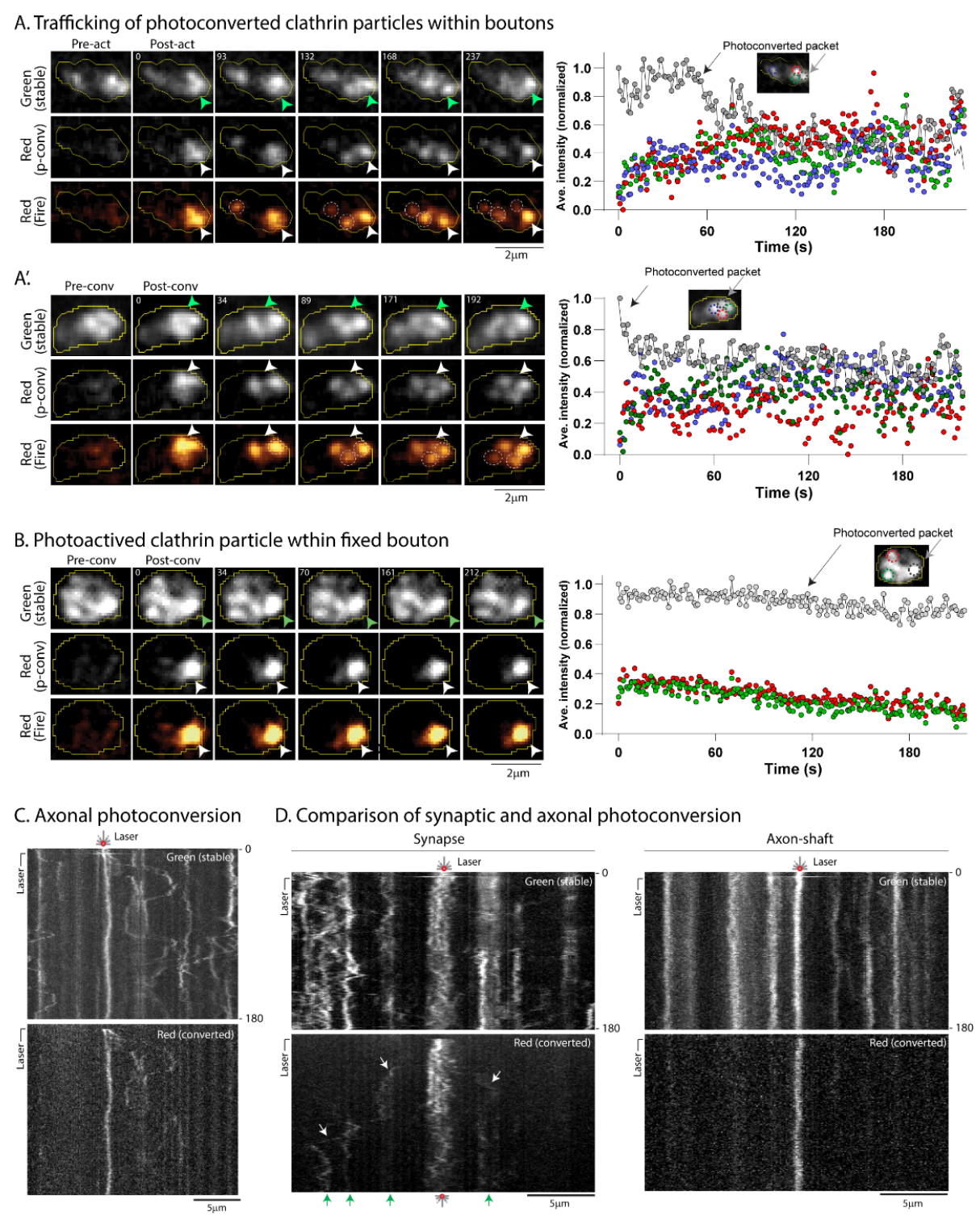

Figure S7: Photoconversion of single clathrin packets in synapses and axons.

(A, A') Photoconversion of single clathrin-packets (Dendra2:CLC) at presynaptic boutons - two more examples. Left: Dendra2:CLC was photoconverted in a single synaptic clathrin-packet (arrowhead, elapsed time in seconds on top left). Note that after photoconversion (middle and bottom panels), the photoconverted packet lost fluorescence, while there was an increase of fluorescence in adjacent packets, identified by stable green fluorescence (some marked by dashed circles in bottom panel). Right: Quantification of intensities in individual packets (normalized to highest post-conversion intensity). Colors in graph correspond to the colors of circles marking packets in inset-image. Note reciprocal fluctuations in photoconverted and non-photoconverted packets.

(B) Same experiment as above, but in fixed NEURONS. Note that while the Dendra2:CLC synaptic packet could be photoconverted, there was little to no loss of fluorescence from this packet, or gain of fluorescence in the other local clathrin packets.

(C) Kymographs from photoactivation of single Dendra2:CLC axonal packets. In this example, note that moving clathrin particles caused some disruption of the photoconverted clathrin, but there was no appreciable loss of clathrin molecules from the photoconverted axonal packet. Also note that the time-scale of dispersion in this case - seconds - is very different from the slower exchange of clathrin molecules at the synapse (over tens of seconds to minutes).

(D) A side by side comparison of synaptic and axonal Dendra2:CLC photoconversion. In the kymographs of photoconverted (red) fluorescence, note that in the case of synapses, single particles of clathrin emerged from the laser-illuminated bouton, and were trapped at adjacent boutons (white arrowheads on kymograph; green arrowheads at bottom mark the positions of non-activated boutons, as determined by the stable green fluorescence). On the other hand, no dispersion of fluorescence was seen when a single axonal clathrin-packet was photoconverted.

Key for kymographs: elapsed time in seconds on right and scale-bar on lower right (laser illumination also marked). 


\section{Figure S8, related to Figure 8}

A. FRB-GFP:CLC colocalizes with endogenous clathrin

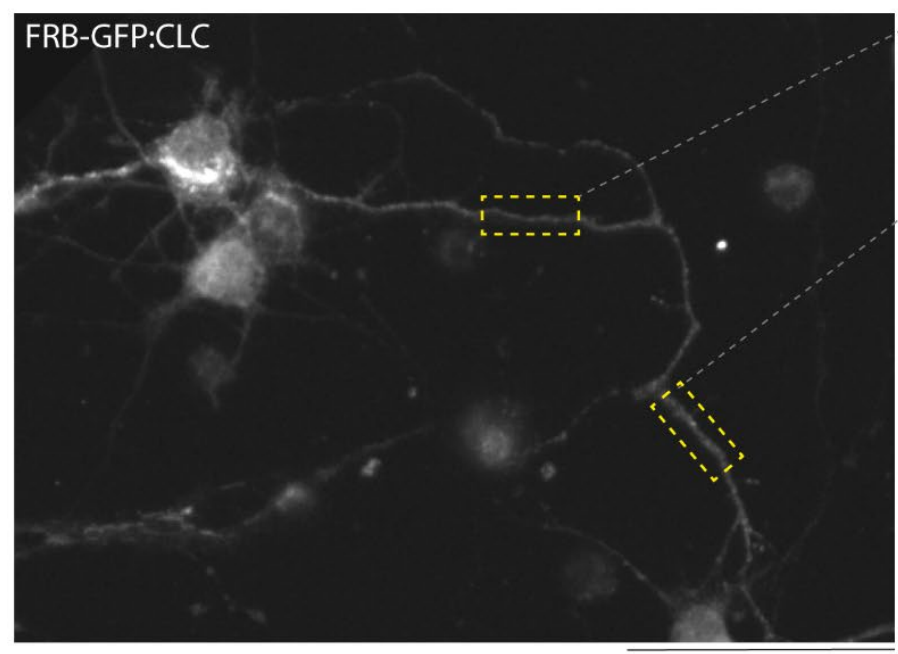

$50 \mu \mathrm{m}$
FRB-GFP:CLC
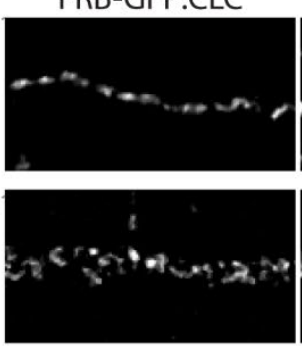

B. FRB-GFP:CLC colocalized with all axonal clathrin

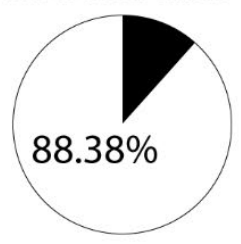

Total Clathrin
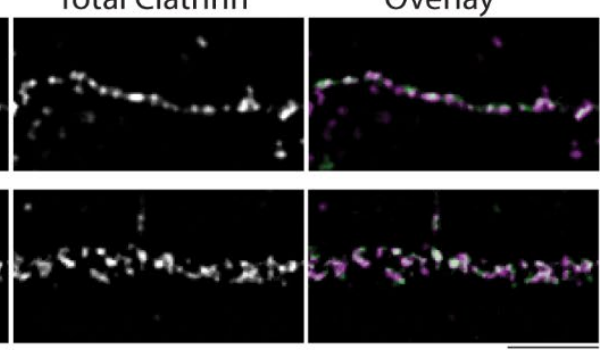

All axonal clathrin colocalized with FRB-GFP:CLC

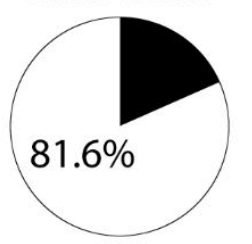

Overlay

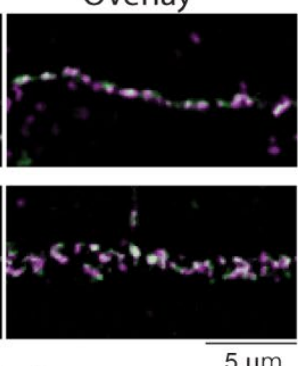

not colocalized

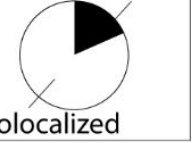

C. Live/dead assay

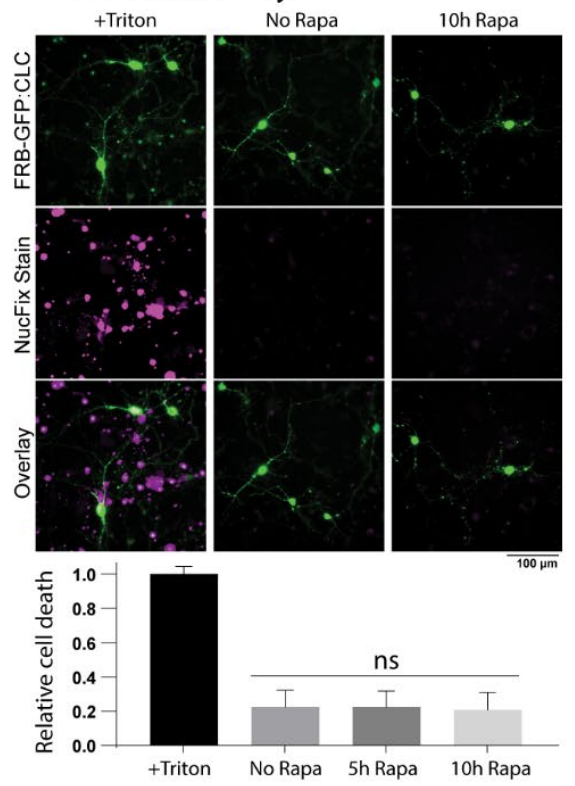

D. Blocking endocytosis attenuates FM4-64 uptake

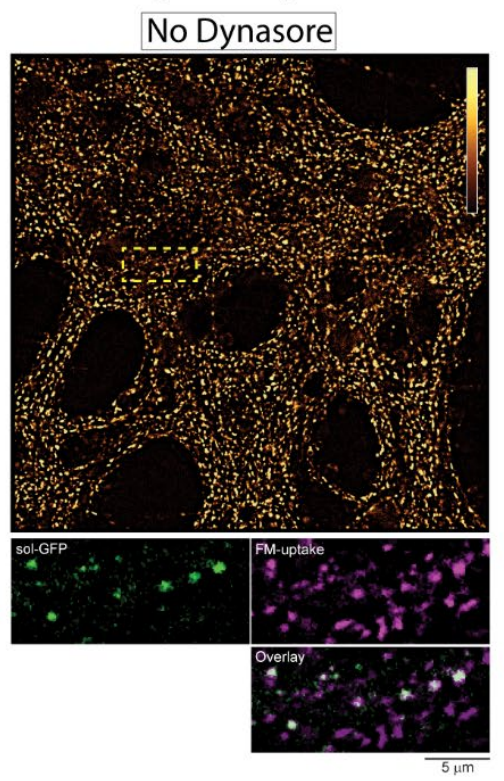

+ Dynasore

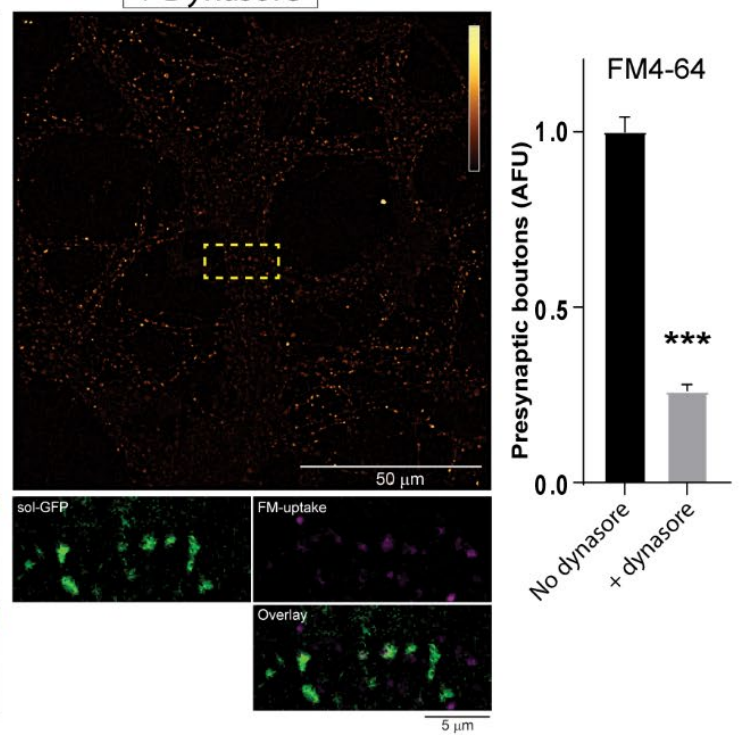

Figure S8: Transfected FRB-GFP:CLC co-assembles with endogenous clathrin.

(A) Neurons were transfected with FRB-GFP:CLC and immunostained with an antibody recognizing the clathrin heavy-chain (see 'methods"). In the insets magnified in A', note the extensive colocalization of transfected FRB-GFP:CLC with endogenous clathrin.

(B) Colocalization analysis indicates that the majority of transfected FRB-GFP:CLC ( 80-90\%) coassembles with the endogenous clathrin in axons ( 250 particles were analyzed from 10 neurons).

(C) Live/dead assay with NucFix stain (see "Methods") was validated in FRB-GFP:CLC transfected neurons by treating the cells with $0.01 \%$ Triton for one minute (left panel). Note that there was no staining above baseline after addition of Rapalog; quantified at bottom (12-16 fields of neurons were imaged for each condition from two separate cultures).

(D) Blocking endocytosis by Dynasore led to an attenuation of the FM4-64 dye uptake, as shown in the representative images on left (zoom from boxed regions shown below) and quantification of the data on right. For quantification, boutons were identified by varicosities in neurons transiently transfected with soluble GFP ( 240-310 synapses were analyzed for each condition from 3 coverslips). 
Table S1. Proteins associated with clathrin - MUDPIT data (related to Fig. 4)

Protein Families
ATP citrate synthase
Actin
Argonaute
Ahnak2
Adaptor Related Protein
Complex
V-ATPase
Bag3
Bcr
Bmp
Calcoco
Ccdc
Clint1
Clathrin
Alpha-crystallin
Casein
Dab2
Connecdenn
Dynamin
Dynein
Edc
Eef
Epsin
Eps
Fatty acid binding protein
Fatty Acid Synthase
Fcho
Fibrinogen A
Filamin A
Gak
Gulp1
H1
Hip1
Hnrnp

Heat shock proteins Intersectin

Kcp

Lactate Dehydrogenase

Lrp1

Maged1

Map7

Myosin

Numb

Osbp

Pdcd

Phgdh

Picalm

Pik3

Prune

Periaxin

Reps

Rundc3a

Sec Faily

Sh3d19

Slc12a4

Snap91

Alpha-synuclein

Sorting nexin

Sos1

Spectrin Beta

Stambpl1

Ston2

Synj1

Tfg

Tnrc6b

Tom112

Trim21

Tubulin

Ubiquitin C

Utrophin

Vcp

\author{
UniProtKB ID (from MudPIT) \\ Q3V117 \\ P68134, P60710, Q8BFZ3, P68033 \\ Q8CJG0 \\ E9PYB0
}

Q8CC13, Q8CBB7, Q3UHJ0, P17426, P17427, Q9DBG3, P84091, P62743, Q9Z1T1

Q9Z1G4

Q9JLV1

Q6PAJ1

Q91Z96

Q8CGU1

D3YZP9

Q5SUH7

Q6IRU5, Q68FD5

P23927

P19228, Q02862

$\mathrm{P} 98078$

Q8K382

P39053, P39054

Q9JHU4

Q3UJB9

P58252

Q80VP1, Q5NCM5

P42567, Q60902

Q05816

P19096

Q3UQN2

E9PV24

Q8BTM8

Q99KY4

Q8K2A1

P15864, P43274

Q8VD75, Q6ZQ77

Q8BG05, Q8VDM6

Q80TZ3, Q71LX8, Q8K0U4, Q61696,

P16627,P17156, Q3U2G2, P48722, P20029,

Q504P4, Q61699

E9Q0N0, B2RR82

Q3U492

P16125

Q91ZX7

Q9QYH6

A2AG50

Q3UH59, Q69ZX3, E9Q174

Q9QZS3, O08919

D3YTT6

Q9WU78

Q61753

Q7M6Y3

Q61194

Q52KR3

O55103

O54916, B9EI38

008576

Q9D1M0, E9QAT4, Q01405

Q91X43

Q9JIS8

Q61548

055042

Q91VH2

Q62245

Q62261

Q76N33

E9PXP7

D3Z656

Q9Z1A1

Q8BKI2

Q5SRX1

Q3U7K7

P68368, Q9CWF2, Q922F4

POCG50

E9Q6R7

Q01853
Gene IDs Acly

Acta1, Actb, Actbl2, Actc1

Ago2

Ahnak2

Ap1b1, Ap1g1, Aak1, Ap2a1, Ap2a2,

Ap2b1, Ap2m1, Ap2s1 , Ap3b1

Atp6v0a1

Bag3

$\mathrm{Bcr}$

Bmp2k

Calcoco1

Ccdc6

Clint1

Cltb, Cltc

Cryab

Csn1s1, Csn1s2a

Dab2

Dennd1a

Dnm1, Dnm2

Dync1h1

Edc4

Eef2

Epn1, Epn2

Eps15, Eps15I1

Fabp5

Fasn

Fcho2

Fga

FIna

Gak

Gulp1

$\mathrm{H} 1-2, \mathrm{H} 1-4$

Hip1, Hip1r

Hnrnpa3, Hnrnpul1

Dnajc6, Hsp90ab1, Hspa12a, Hspa1a

Hspa1l, Hspa2, Hspa4, Hspa4I, Hspa5,

Hspa8, Hsph1

Itsn1, Itsn2

Kcp

Ldhb

Lrp1

Maged1

Map7d2

Myh10, Myh11, Myo6

Numb, Numbl

Osbpl3

Pdcd6ip

Phgdh

Picalm

Pik3c2a

Prune2

Prx

Reps1, Reps2

Rundc3a

Sec13, Sec16a, Sec23a

Sh3d19

Slc12a4

Snap91

Snca

Snx9

Sos1

Sptbn1

Stambpl1

Ston2

Synj1

Tfg

Tnrc6b

Tom112

Trim21

Tuba4a, Tubb2b, Tubb6

Ubc

Utrn

Vcp
Organism

Mus musculus (Mouse)

Mus musculus (Mouse)

Mus musculus (Mouse)

Mus musculus (Mouse)

Mus musculus (Mouse)

Mus musculus (Mouse)

Mus musculus (Mouse)

Mus musculus (Mouse)

Mus musculus (Mouse)

Mus musculus (Mouse)

Mus musculus (Mouse)

Mus musculus (Mouse)

Mus musculus (Mouse)

Mus musculus (Mouse)

Mus musculus (Mouse)

Mus musculus (Mouse)

Mus musculus (Mouse)

Mus musculus (Mouse)

Mus musculus (Mouse)

Mus musculus (Mouse)

Mus musculus (Mouse)

Mus musculus (Mouse)

Mus musculus (Mouse)

Mus musculus (Mouse)

Mus musculus (Mouse)

Mus musculus (Mouse)

Mus musculus (Mouse)

Mus musculus (Mouse)

Mus musculus (Mouse)

Mus musculus (Mouse)

Mus musculus (Mouse)

Mus musculus (Mouse)

Mus musculus (Mouse)

Mus musculus (Mouse)

Mus musculus (Mouse)

Mus musculus (Mouse)

Mus musculus (Mouse)

Mus musculus (Mouse)

Mus musculus (Mouse)

Mus musculus (Mouse)

Mus musculus (Mouse)

Mus musculus (Mouse)

Mus musculus (Mouse)

Mus musculus (Mouse)

Mus musculus (Mouse)

Mus musculus (Mouse)

Mus musculus (Mouse)

Mus musculus (Mouse)

Mus musculus (Mouse)

Mus musculus (Mouse)

Mus musculus (Mouse)

Mus musculus (Mouse)

Mus musculus (Mouse)

Mus musculus (Mouse)

Mus musculus (Mouse)

Mus musculus (Mouse)

Mus musculus (Mouse)

Mus musculus (Mouse)

Mus musculus (Mouse)

Mus musculus (Mouse)

Mus musculus (Mouse)

Mus musculus (Mouse)

Mus musculus (Mouse)

Mus musculus (Mouse)

Mus musculus (Mouse)

Mus musculus (Mouse)

Mus musculus (Mouse)

Mus musculus (Mouse)

Mus musculus (Mouse)

Mus musculus (Mouse) 
Ybx

Ywhag

FLJ45252 homolog
P62960, Q9JKB3

P61982

Q6PIU9
Ybx1, Ybx3

Ywhag

FLJ45252 homolog musculus (Mouse)

Mus musculus (Mouse)

Mus musculus (Mouse) 